\title{
Microstructure and macroscopic properties of polydisperse systems of hard spheres
}

\section{Vitaliy Ogarko}

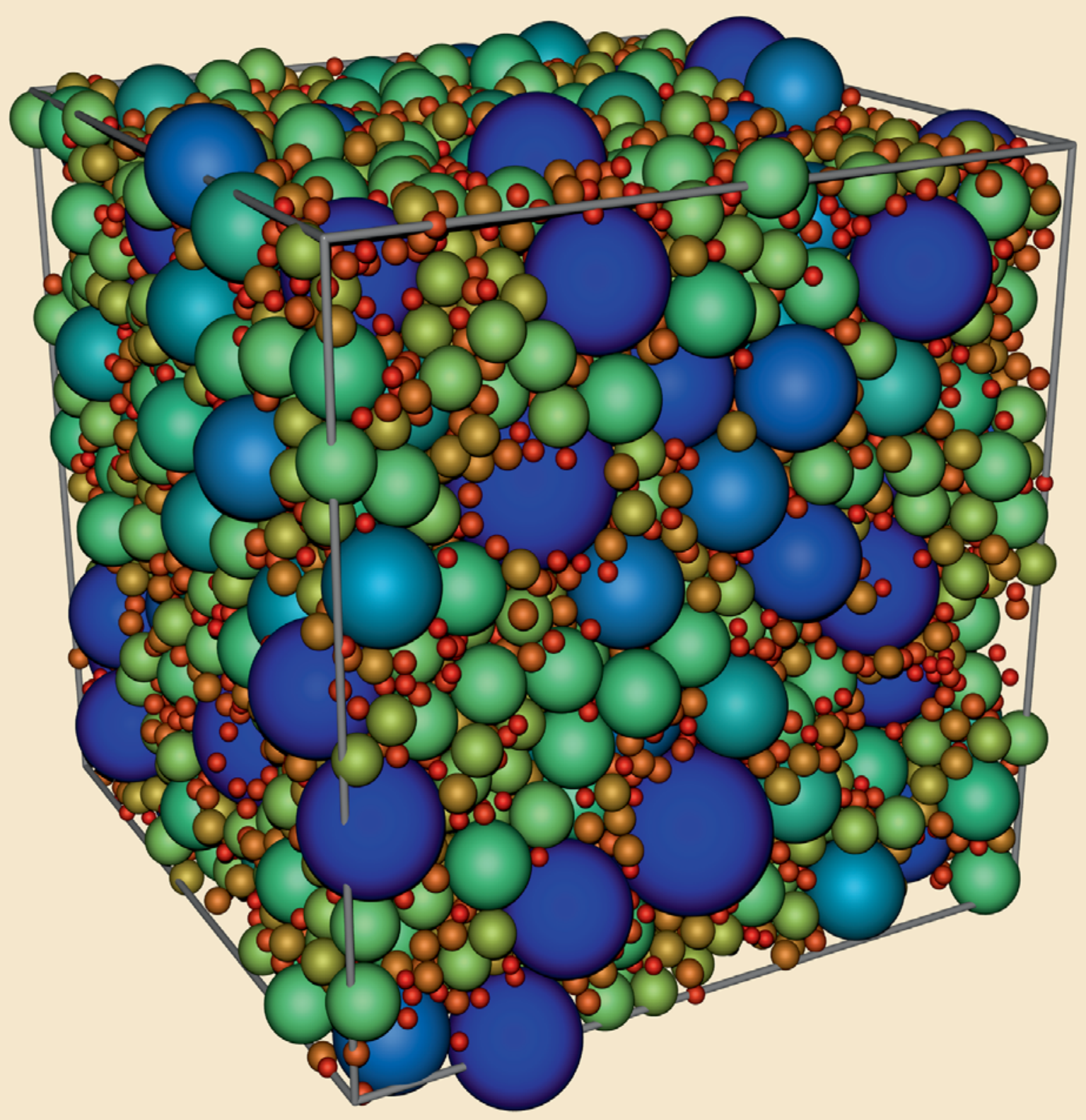


MICROSTRUCTURE AND MACROSCOPIC PROPERTIES OF POLYDISPERSE SYSTEMS OF HARD SPHERES

Vitaliy Ogarko 


\section{Composition of the graduation committee:}

Prof. dr. Geert P. M. R. Dewulf (chairman)

Prof. dr. rer.-nat. Stefan Luding (supervisor)

Prof. dr. Wim J. Briels

Prof. dr. Jens D. R. Harting

Dr. ir. Ronald G. K. M. Aarts

Prof. dr.-ing. Holger Steeb

Prof. dr. Andrés Santos

Dr. Till Kranz
Univeristy of Twente Univeristy of Twente Univeristy of Twente Univeristy of Twente Univeristy of Twente Ruhr-University Bochum University of Extremadura University of Göttingen, University of Oxford

The work in this thesis was carried out at the Multi Scale Mechanics group, MESA+ Institute for Nanotechnology, Faculty of Science and Technology (CTW) of the University of Twente, Enschede, The Netherlands.

ISBN 978-90-365-3669-1

DOI: $10.3990 / 1.9789036536691$

Official URL: http://dx.doi.org/10.3990/1.9789036536691

Printed by Gildeprint Drukkerijen, Enschede, The Netherlands.

Copyright (c) 2014 by Vitaliy Ogarko, The Netherlands.

All rights reserved. No part of the material protected by this copyright notice may be reproduced or utilized in any form or by any means, electronic or mechanical, including photocopying, recording or by any information storage and retrieval system, without written permission of the author. 


\title{
MICROSTRUCTURE AND MACROSCOPIC PROPERTIES OF POLYDISPERSE SYSTEMS OF HARD SPHERES
}

\author{
DISSERTATION
}

\author{
to obtain \\ the degree of doctor at the University of Twente, \\ on the authority of the rector magnificus, \\ prof. dr. H. Brinksma, \\ on account of the decision of the graduation committee, \\ to be publicly defended \\ on Friday the $2^{\text {nd }}$ of May 2014 at 14:45
}

by

Vitaliy Anatolyevich Ogarko born on May $5^{\text {th }} 1985$

in Vladivostok, Russia 
This dissertation has been approved by Prof. dr. rer.-nat. Stefan Luding 
I dedicate this dissertation to my family and friends. 



\section{Overview}

This dissertation describes an investigation of systems of polydisperse smooth hard spheres. This includes the development of a fast contact detection algorithm for computer modelling, the development of macroscopic constitutive laws that are based on microscopic features such as the moments of the particle size distribution, and the development of new analysis techniques to study microstructure in such systems with different physical behaviour, i.e., gaseous, liquid, glassy and crystalline states.

The first chapter gives a general introduction to the themes and topics treated in this dissertation.

In the second chapter I deal with the numerical problem of contact detection among arbitrarily polydisperse objects. I present a new efficient algorithm for contact detection which even increases performance with increasing degree of polydispersity. The performance of the algorithm is theoretically analyzed for various particle size distributions and volume fractions, and recommendations are given concerning the choice of optimal algorithm parameters.

The third chapter focuses on the theoretical prediction of the equation of state and the jamming density. The equilibrium equation of state of a fluid mixture of polydisperse hard spheres is well described by considering only the first three moments of the size distribution function. Consequently, the (thermodynamic) properties of a polydisperse fluid can be reproduced by a well-chosen "equivalent" bidisperse fluid with the same three moments. In this study I ask the question: How many moments are needed to predict the pressure and the jamming density of polydisperse mixtures in compressed non-equilibrium glassy states? I find that five moments suffice to describe the properties of polydisperse mixtures for all densities, including glassy, nonequilibrium states and the maximal jamming density. Hence, as proposition, polydisperse mixtures can be modelled by a well-chosen tridisperse system.

In the fourth chapter I suggest a new way to characterize the microstructure in mono- and polydisperse hard-sphere systems, based on the local correlation of four particle positions. This analysis allows to distinguish between gaseous, liquid, partially and fully crystallized, and glassy (random) jammed states. A common microstructural feature is observed in crystalline and glassy jammed states, suggesting the presence of "hidden" two-dimensional order in polydisperse random close packings of three-dimensional spheres.

Finally, conclusions and outlook close the dissertation. 



\section{Samenvatting}

Dit proefschrift doet verslag van een onderzoek naar systemen van polydisperse (verschillend in grootte) gladde, harde bolletjes. Onderdelen hiervan zijn de ontwikkeling van een snel algorithme voor contact detectie in computersimulaties; de ontwikkeling van macroscopische constitutieve wetten die zijn gebaseerd op microscopische kenmerken zoals de momenten van de verdeling van de deeltjesdiameters; en de ontwikkeling van nieuwe analyse technieken voor het bestuderen van de microstructuur in zulke systemen met verschillend fysisch gedrag, namelijk de gasvormige, vloeibare, glas-achtige en kristallijne aggregatietoestanden.

Het eerste hoofdstuk is een algemene inleiding in de thema's en onderwerpen die in dit proefschrift worden behandeld.

In het tweede hoofdstuk behandel ik het numerieke probleem van contact detectie tussen willekeurig polydisperse objecten. Ik presenteer een nieuw efficint algorithme voor contact detectie, waarvan de performance zelfs verbetert bij een toenemende graad van polydispersiteit. De performance van het algorithme is theoretisch geanalyseerd voor verschillende deeltjesdiameter verdelingen en volume fracties, en er worden aanbevelingen gegeven met betrekking tot de keuze van optimale parameters voor het algorithme.

Het derde hoofdstuk spitst zich toe op de theoretische voorspelling van de toestandsvergelijkingen en de "jamming" dichtheid (blokkeringsdichtheid). De evenwichts toestandsvergelijking van een vloeibaar mengsel van polydisperse harde bolletjes wordt goed beschreven in termen van de eerste drie momenten van de verdelingsfunctie van deeltjesdiameters. Dientengevolge kunnen de (thermodynamische) eigenschappen van een polydisperse vloeistof worden gereproduceerd door een welgekozen "equivalente" bidisperse vloeistof met dezelfde drie momenten. In dit onderzoek stel ik de vraag: hoeveel momenten zijn nodig om de druk en "jamming" dichtheid van polydisperse mengsels in de samengeperste niet-evenwichts glas-achtige toestanden te voorspellen? Ik concludeer dat vijf momenten volstaan om de eigenschappen van polydisperse mengels te beschrijven voor alle dichtheden, ook de glazen, nietevenwichtstoestanden en de maximale "jamming" dichtheid. Daardoor, als een voorspelling, kunnen polydisperse mengsels door een goedgekozen tridispers systeem worden gemodelleerd.

In het vierde hoofdstuk stel ik een nieuwe methode voor om de microstructuur van mono- en polydisperse systemen van harde bolletjes te karakteriseren, gebaseerd op de locale correlatie van de posities van vier deeltjes. Met 
deze analyse kunnen gasvormige, vloeibare, gedeeltelijk en volledig kristallijne, en glazen (wanordelijke) "jammed" toestanden worden onderscheiden. Een gemeenschappelijk microstructureel kenmerk is waargenomen in kristallijne en glas-achtige "jammed" toestanden, wat duidt op de aanwezigheid van een "verborgen" twee-dimensionale orde in polydisperse wanordelijke dichte pakkingen van drie-dimensionale bolletjes.

Het proefschrift eindigt met conclusies en een vooruitblik. 


\section{Acknowledgements}

When I was a bachelor student, I applied for a PhD position in the Netherlands, and have not received any positive answer. Then I decided to first finish my master study looking forward to more opportunities in the Netherlands. During my master study I was first time in my life involved into research, thanks to my supervisor Prof. Valeriy Il'in. He showed to me how interesting and challenging research can be. His attitude of working always reminds me the science fantasy novel "Monday Begins on Saturday" by Strugatsky brothers. After graduation I moved to Japan for an internship at Schlumberger. There I met many intelligent people that inspired me to apply for a PhD study. I still remember my interview with Stefan by Skype from Japan, in that time I did not understand well the meaning of words like jamming or multi-scale, and had very limited knowledge about research and modelling. Now, I understand well what those words mean and I enjoyed a lot working on variety of research topics, over the last years, while also having lots of fun with my friends and colleagues. At this point, I want to express my deepest gratitude to the people who are involved in the successful completion of my $\mathrm{PhD}$.

I would like to thank my supervisor Prof. Stefan Luding for the belief he has in me. Stefan, thank you for giving me absolute freedom to work on different interesting problems, for giving opportunity to travel to interesting places, for our uncountable discussions about science and life, and for helping me with any kind of problems. You showed me the world of computer simulations in physics, how to perform independent research and write scientific papers. I have learned a lot from you, both professionally and personally. I will also not forget your wife, Gerlinde - who were organizing group events. Thank you both for your help to me and Masami, and for dinners we had together.

Then I would like to thank Sylvia, the group secretary. Sylvia, thank you for always being extremely helpful, for your care and support you showed to me. Thank you for all talks, jokes and fun we had together. Thank you for being always super kind with me and trying to solve any kind of problems I and Masami had. I will be missing you.

I would also like to thank my committee members for their interest in my research. In particular, I am very thankful to Prof. Andrés Santos, for helping me during my $\mathrm{PhD}$ with many valuable advices. Till, thank you for introducing to me inelastic particles and for productive collaboration. I would 
also like to thank professors John Finney and Leslie Woodcock, with whom I had fruitful scientific discussions during their visit of the MSM group. The financial support from the Dutch Technology Foundation STW is gratefully acknowledged.

In no particular order, then, I would like to thank my former and present colleagues in the MSM group - Sebastián for wine parties and help with writing of my first journal paper, Fatih for talks on religion, Kazem for jokes and dinners, Saurabh for science and career talks, Abhi and Ankit for beer talks on many subjects, Mateusz for showing me Poland, Kay for the great time we spent in Barcelona, Nishant for being a great friend with whom we had many discussions about all kinds of everything, and for the memorable time we spent together in Enschede and San Francisco, Rohit for the wolves and talks, Nico and Dinant for productive work together that led to two journal papers, Vanessa for teaching me how to make nice slides for presentations, Kuni and Megumi for many parties, dinners and talks at your house, Wouter den Breeijen for helping with cluster, Wouter den Otter for helping with math, Thomas, Remco, Anthony, Martin, Sudheshna, Deepak, Brian, and Katia for bringing the friendly and creative atmosphere. I would also like to thank Barend Gehrels, Dinant Krijgsman, and Wouter den Otter for taking out your time for helping with the samenvatting of this thesis. To my friends in Enschede and beyond - Franklin, Taras, Juan, Kostya and Kristina, Ken and Kaori, Artem and Natasha, Sergey, Eugeniy, Stas, Ivan and Veronika, Dmitriy Hazin, Dmitriy Pavlitskiy, Anton Kulchitsky, Misha Belonosov, Maxim Protasov, and Ilya Silvestrov - thank you all.

Finally, I wholeheartedly thank my family. I thank my mother Galina for all sacrifices, prayers, encouragement and support during my years of education. I thank my grandmother Anna for her unconditional love and all the joy she brought to my life, undoubtedly, her memory will be with me always, I am sure, I have made you very proud. Special thanks to my lovely girlfriend Masami, whom I met in Japan during the internship. Masami, you have shown nothing but love and care during our time in Japan and Netherlands, and when we were far apart. Thank you for always being there for me throughout the course of this work.

To everyone who contributed to my life, whose name I unfortunately forgot to include above, you mean no less to me. I appreciate you all.

Vitaliy Ogarko

Amsterdam, April 2014. 


\section{Contents}

1 Introduction 1

1.1 General motivation . . . . . . . . . . . . . . . . . . 1

1.1.1 Why hard spheres? . . . . . . . . . . . . . . . 1

1.1.2 Why polydisperse? . . . . . . . . . . . . . 2

1.1.3 Types and effects of polydispersity . . . . . . . . . 3

1.2 Challenges . . . . . . . . . . . . . . . . . 4

1.2.1 Numerical simulations . . . . . . . . . . . . . . . 4

1.2 .2 Theory . . . . . . . . . . . . . . . 5

1.2.3 Microstructural description . . . . . . . . . . 6

1.3 Computer simulations . . . . . . . . . . . . . . 6

1.3.1 Event-Driven Molecular Dynamics . . . . . . . . . . . 7

1.3.2 Discrete Element Method . . . . . . . . . . . . 8

1.4 Thesis structure . . . . . . . . . . . . . . . . . . . 8

2 Contact detection of arbitrarily polydisperse objects 11

2.1 Algorithm description . . . . . . . . . . . . . . . 11

2.1 .1 Introduction . . . . . . . . . . . . . . . . 11

2.1 .2 Algorithm . . . . . . . . . . . . . . . . . 12

2.1.3 Selection of the optimal grid parameters . . . . . . 16

2.1.4 Numerical experiments . . . . . . . . . . . . . . . 18

2.1.5 Summary and Conclusions . . . . . . . . . . . . . 22

2.2 Performance analysis . . . . . . . . . . . . . . . . . . 25

2.2 .1 Introduction . . . . . . . . . . . . . . . . 25

2.2 .2 Algorithm . . . . . . . . . . . . . . . . . . . . . . . 29

2.2 .3 Performance analysis . . . . . . . . . . . . 33

2.2.4 Particle size probability distribution functions . . . . . 37

2.2 .5 Cell-sizes distribution . . . . . . . . . . . . . . . 38

2.2.6 Summary and Conclusion _. . . . . . . . . . . . 49

2.2 .7 Recommendations . . . . . . . . . . . . . . . 50

3 Equation of state and jamming density $\quad 55$

3.1 Poly- versus bi-disperse systems . . . . . . . . . . . . 55

3.1 .1 Introduction . . . . . . . . . . . . . . 55

3.1 .2 Theory . . . . . . . . . . . . . . . . . 56

3.1.3 Comparison with simulations . . . . . . . . . . . 59

3.1.4 Summary and Conclusions . . . . . . . . . . . . 72 
3.2 Poly- versus tri-disperse systems . . . . . . . . . . . . . 80

3.3 Supplementary materials . . . . . . . . . . . . . . . . . 88

3.3.1 Details on the event-driven simulations . . . . . . . 88

3.3.2 Size distribution parameters . . . . . . . . . . . . . . 89

3.3.3 Measuring bond-orientational order . . . . . . . . . . 96

3.3.4 Structure factor and spectral density . . . . . . . . . 98

4 Structure characterization of hard sphere packings 105

4.1 Introduction . . . . . . . . . . . . . . . . 105

4.2 Method of analysis . . . . . . . . . . . . . . . 107

4.3 Simulation details . . . . . . . . . . . . . . . . . . . . 109

4.4 Results and discussion . . . . . . . . . . . . . . . . 110

4.4.1 Crystallization path . . . . . . . . . . . . 110

4.4 .2 Glassification path . . . . . . . . . . . . . . . . 112

4.4 .3 Polydisperse systems . . . . . . . . . . . . . . . 114

4.5 Conclusions . . . . . . . . . . . . . . . . . 115

5 Conclusions and Outlook 117 


\section{Chapter 1}

\section{Introduction}

\subsection{General motivation}

This dissertation presents the results of combined computational and theoretical studies on systems of frictionless impenetrable objects, henceforth, hard particles. In a three-dimensional Euclidean space, the particles occupy a certain volume (or packing) fraction $\nu$ of the total volume. We focus on systems where all particles have the same spherical shape, but may be very different in size. This variation in size is referred to as polydispersity. We study such polydisperse systems for very different volume fractions, starting from the dilute gaseous state up to the dense jammed packings, where the particles are packed so tightly that they cannot move freely due to the impenetrability constraint.

\subsubsection{Why hard spheres?}

Systems of hard particles interacting only with infinite repulsive pairwise forces on contact are models of more complex many-body systems when repulsive interactions are the primary factor in determining their structure. In dense matter the structure is determined first of all by impenetrability of particles (e.g., atoms), and ultimately comes from geometric properties of the packing-structure of nonoverlapping spheres in three-dimensional space [1-4]. Hard-particle systems are therefore widely used as models for colloids [5], granular materials [6-8], glasses [9], liquids [2], and other random media [4]. The hard-sphere model successfully reproduces the main structural properties of the condensed phase, such as crystallization or melting of liquids [10-12] and amorphous-to-solid phase transition $[13,14]$. Furthermore, hard-sphere packings have inspired mathematicians and been the source of numerous challenging (many still open) theoretical problems [15-18], such as the identification of packing structures with extremal properties (e.g., the lowest or 


\section{Introduction}

highest density jammed packings), the quantification of disorder via order metrics, and others. The results we obtain can have practical applications such as for the production of advanced high-performance materials (ceramics, metals, plastics, composites, concrete, etc.) [4], helping to develop better constitutive equations for the mechanical behavior of granular media, and other particle based materials.

The simplicity of the hard sphere model allows to focus on its most fundamental aspect, which is essentially geometrical, due to the excluded volume. It is hard to think of a simpler physical system which displays all the phenomenology analogous to that of simple gases and fluids, as also jammed, glassy and solid states [19].

Systems of frictionless and hard spheres cannot be realized experimentally. However, colloids and granular materials are very good approximations of hard spheres. They can be synthesized either by tuning the interaction potential between the colloidal particles, by e.g. coating colloidal spheres with poly-12-hydroxystearic acid [20], or by using manufactured particles such as ball bearings, marbles, beads, etc., to make them hard [21]. The larger the confining stress, the worse the assumption "hard" becomes. Furthermore, friction can be reduced by lubrication as in colloids or wet granular media.

\subsubsection{Why polydisperse?}

In a sample of pure water all molecules of $\mathrm{H}_{2} \mathrm{O}$ are identical. This is usually not the case for particles in soft matter. A collection of mesoscopic particles will almost always exhibit some continuous variation in properties such as size, charge, shape or chemical makeup. The majority of particle systems found in industrial and natural processes are composed of particles of a broad range of particle sizes. This variation hugely complicates their study, introducing additional peculiar phenomena and necessitating a more sophisticated theoretical treatment, when compared to ideal monodisperse (i.e., non-polydisperse) systems.

Whether colloidal or granular particles are artificially prepared or not, in the best case the particle size distribution is still a narrow distribution around an average size. It is obvious that even such a narrow polydispersity can influence the physics and mechanical properties of these systems. By the use of polydisperse mixtures, it is possible to create systems with a behavior that cannot be described by the simple monodisperse-like approximation. For example, polydispersity induces the shift of the critical temperature and density, as was illustrated for a polydisperse generalization of the van der Waals model [22].

In theoretical studies polydispersity is often ignored, as solving the singlesized or monodisperse problem is quite complicated. However, as real exper- 
iments are always performed on at least slightly polydisperse systems, it is essential to take the effects of polydispersity properly into account.

\subsubsection{Types and effects of polydispersity}

The effect of polydispersity on the phase behavior of hard spheres has been investigated by experiments, computer simulations, density functional theories, and simplified analytical theories.

Conceptually, in literature, two types of polydispersity can be distinguished [23]: One is present in multi-component mixtures and the other one in self-assembling systems. The first, "intrinsic polydispersity", arises from the fact that the particles present in the system are different by construction (in size, charge, or any other feature) and their characteristics are not changed by the interaction with other particles. This kind of system is like multi-component mixtures in which, at least in principle, the composition can be externally imposed. Significant size polydispersity should destabilize the crystal phase [24], because it is difficult to accommodate a range of diameters on a lattice structure. Experiments have indeed shown that crystallization is suppressed above a terminal polydispersity $\delta_{t} \approx 0.12$ in $3 \mathrm{D}[25,26]$, defined as the standard deviation of the diameter distribution normalized by its mean, as supported by numerics [27]. Since then, much theoretical work has focused on estimating $\delta_{t}$; for details see Ref. [28].

Another new phenomenology has its origin in fractionation into phases with different compositions, e.g., sizes [29-32] and their coupling with other phenomena already present in a monodisperse system [33,34]. Fractionation can lead to solid-solid coexistence [28-30,35], where a broad diameter distribution is split into a number of narrower ones, separated in space. This occurs because the loss of entropy of mixing is outweighed by the better packing efficiency, and therefore the reduction in excess free energy, of crystals with narrow size distribution [28,35]; accordingly, as the overall polydispersity of the system grows, the number of possible coexisting solid phases is expected to increase. This rich behaviour is suggested already by Gibbs' phase rule, where with an infinite number of particle species present there is a priori no limit for the number of phases.

Extensive computational and experimental research has shown that, compared to the monodisperse case, hard-sphere systems with sufficient size polydispersity tend to remain amorphous over a broad range of volume fractions. Emergence of order by crystallization (i.e., crystal nucleation) is strongly suppressed $[21,27,36]$. For polydisperse systems with a continuous distribution of particle radii, the phase diagram and the existence and structure of thermodynamic crystal and glassy phases is still actively debated.

In Ref. [28], Fasolo et al. discuss the reentrant melting found by Bartlett 


\section{Introduction}

and Warren [33]: for polydispersity $\delta$ just below $\delta_{t}$ they predict that compressing a crystal might transform it into a fluid. While in the monodisperse case the solid has the lowest free energy at all volume fractions above $\nu \approx 0.55$, the fluid can become preferred again at larger $\nu$, if polydispersity is large enough. Polydispersity reduces the maximum volume fraction in a crystal (since a range of diameters need to be accommodated on uniformly spaced lattice sites), whereas it could increase the maximum volume fraction in the disordered fluid state, where smaller spheres can fill "holes" between larger particles.

In granular materials, the degree of polydispersity strongly influences their mechanical response to external loads during shear [37] or compaction [38-40], as well as segregation and flow behaviour during mixing [41,42] and discharge processes [43]. (Note that in Ref. [41] also binary and ternary mixtures were studied using both experiments and simulations - we discuss them later in Chapter 3.) Microstructural characterization of polydisperse particulate media is critical to understand and predict macroscopic properties of soft and granulate systems [4].

Blaak et al. [23] describe the second kind of polydispersity, which can be found in self-assembling systems [44] (surfactants forming micelles/vesicles, monomers forming chains or structures like agglomerates, etc.) as follows: The aggregates present in such systems can be identified as polydisperse particles, each with different size, shape, conformation, etc. The difference with the intrinsically polydisperse systems is that the composition is determined by the chemical equilibrium between the constituents of the aggregates, which can be identical: for example, polydisperse agglomerates can be composed of identical, monodisperse primary particles. As a consequence, no fractionation is to be expected, since it would lead to new equilibrium. In principle, the system can compensate losses of entropy by adjusting its composition. There are, however, other constraints in the system (the number of primary, small constituents, for instance), and these may induce new kinds of transitions characterized by the appearance of one or a few macroscopic aggregates. Such are phenomena as the appearance of lamellar and columnar phases in surfactant solutions [45], emulsification failure in micro-emulsions [46], or long chain formation in polymer solutions [47].

\subsection{Challenges}

\subsubsection{Numerical simulations}

A study of polydisperse mixtures is far from trivial. In the case of intrinsic polydispersity there is the experimental problem of how to fabricate colloidal 
particles according to a desired particle size distribution. Although in simulations this seems to be somewhat under better control, one could easily run into the problem of finite size effects due to an insufficient or inadequate sampling of particle sizes. (This is not the case in self-assembling systems, although, experimentally, their polydispersity cannot be easily controlled and characterized since it can change.) If a particle mixture is highly polydisperse, the simulation of a packing needs a very large sample of particles from the given grain size distribution in order to reproduce the size relations of the mixture within the sample. For example [48], in a typical concrete mixture particle sizes vary from less than $0.1 \mu \mathrm{m}$ up to $200 \mu \mathrm{m}$ with even larger particles up to a few centimeter if aggregates such as gravel are added. A representative sample must contain many millions or even billions of small particles surrounding a single big particle. Highly efficient algorithms and data structures, for, e.g., fast contact detection, are needed to simulate systems of such huge particle numbers, even if we restrict ourselves to simple spherical particles of different sizes. We address this challenge in Chapter 2.

\subsubsection{Theory}

Theoretical descriptions of polydisperse systems are mainly based on a small set of moments of the particle size distribution [31,32,49-54]. In order to reduce the strictly infinite number of equations for polydisperse systems to a finite set, Sollich, Cates and Warren [50,51] have assumed that the excess free energy in a polydisperse hard-sphere mixture depends only on a limited set of moment densities of the diameter distribution. The price for this simplification is that only approximate results for phase coexistence are obtained if finite amounts of several phases coexist [35]. Within scaled particle theory the pressure of a mixture of hard spheres may be shown readily to be an explicit function of just three diameter moments [55]. The same simplification is evident also in the approximate equation of state obtained from the PercusYevick closure for a system of polydisperse hard spheres [56]; also in the case of the "improved" equation of state obtained by Boublik [57] and Mansoori et al. [58] from an interpolation between the Percus-Yevick virial and compressibility equations; and also in A. Santos et al.'s approaches [59-61].

One of the promising features of a "finite moment" assumption is that is allows the properties of a polydisperse system to be "mapped" onto those of a much simpler e.g. binary mixture. This seems to be a rather successful approach for equilibrium fluids and slightly polydisperse crystals [49,52]. It is not known if the finite moment approximation is reasonable at all densities and can be applied to very polydisperse or dense, glassy, non-equilibrium mixtures. We address this challenge in Chapter 3. 


\subsubsection{Microstructural description}

First studies of disordered hard sphere packings were performed by Bernal on mechanical packings of steel balls [1]. He noted that a disordered packing has a limiting (critical) density, i.e., a packing with higher density inevitably contains crystalline regions. Physical experiments give an estimate of $0.637-0.64$ for this density $[1,62,63]$, computer simulations provide $0.637-$ $0.649[13,14,64-67]$. This density is substantially lower than the maximum packing fraction attainable in the densest crystalline structures, and depends on history [68], and other material parameters. The origin of this critical density for disordered packings and its precise value are unknown today. It is unclear which geometrical principles are at work in disordered packings and why density is limited at about 0.64 . This problem still remains a challenge for both physicists and mathematicians $[1,69,70]$.

Another open question is what microstructural properties in glasses (i.e., amorphous random dense packings) are different from those found in liquids [71]. How these structural properties are involved in the glass transition? Why glasses get so viscous? Might there be some hidden structure? If so, questions are how to determine that structure and what size and type is that structure [72]?

One of the most widely used tools to investigate the structure of isotropic random packings is the pair-correlation function, which is the Fourier transform of the experimental wavelength-dependent x-ray- (or neutron-) scattering intensity $[73,74]$. This quantity gives interesting information on the sphere correlations, but is averaged too much to describe in detail the topology of the local structure; other methods to get local information are mainly based on the Voronoi tessellation $[14,69,70,75]$. We suggest that answers to these questions can be sought in the study of the local multi-particle structure of mono- and polydisperse systems of hard spheres. We address this challenge in Chapter 4.

\subsection{Computer simulations}

As the experimental methods do not provide all the essential insight into the micromechanical properties of particulate assemblies, theoretical and computational approaches are used to understand colloids or granular media. The use of computer simulations has since long helped to study where the experiments cannot go. In mechanics and physics two ways to describe and model particulate, inhomogeneous materials like powders or grains may be distinguished [76]. The first approach, based on continuum theory, relies on empirical assumptions about the constitutive macroscopic material behaviour. The macroscopic approach can be complemented by a second microscopic 
description of the material, where the particles and their interactions are modelled one by one. The former approach involves stress, strain and plastic yield conditions, whereas the latter deals with local interaction laws for each contact. In this thesis we focus on the microscopic approach and aim to provide macroscopic constitutive laws that are based on microscopic features such as the moments of the particle size distribution.

There are mainly two methods for the microscopic simulation of colloids and granular matter. The first assumes perfectly rigid spheres that interact via instantaneous collision, realising the assumption of binary interaction as in kinetic theory. This method is known as event-driven since the simulation advances through the events, such as collisions.

On the other hand, one can model the grains as soft particles that interact via a given interaction force. This thesis addresses both approaches. We use the event-driven method to study the role of various wide particle size distributions on the equation of state and the jamming density. The elastic soft-sphere model is used to test our new contact detection algorithm. Next, we introduce both models and the codes we have used in this thesis.

\subsubsection{Event-Driven Molecular Dynamics}

Discrete infinitely steep potentials have played a major role in the development of early molecular dynamics simulation methods. The very first molecular dynamics simulations were performed with discrete potentials because of their relatively high computational efficiency. Recent advances in the simulation of discrete potential systems have allowed the construction of molecular dynamics algorithms that scale linearly with the system size [77], allowing access to large system sizes and long time scales. Discrete (multi-step) potentials can even accurately approximate soft potentials, with possible savings in computational cost and time. The simplest discrete potential is the hardsphere model: the interaction energy is zero if particles are not in contact, and infinite if they are, hence creating instantaneous and binary collisions. A collision is called an event, since the state (velocity) of the particles changes discontinuously.

Event-driven means that the state of the system evolves in time from one event to the next. Since the dynamics between events can be solved analytically, the integration of the equations of motion is processed as a sequence of events rather than by fixed, small time-steps. After each event, the time of the next event in the system is calculated and then the system correspondingly advances to this time. In brief, the algorithm consists of:

1. Given the times, positions and velocities of all particles in the system,

2. predict the time of the next collision, 


\section{Introduction}

3. advance the time of the involved partners to that instant,

4. carry out the collision with a given collision rule, and

5. update the velocities of the particles that collided;

6. repeat from 1 .

For the details of the algorithm, we refer the reader to Chapter 3 together with standard papers and books, see e.g. Refs. [78-80].

In this thesis, we use a modification of A. Donev's code [21] to account for different particle size distributions. This code implements a modification of the Lubachevsky-Stillinger algorithm [81], which allows the diameter of the particles to grow in time with a constant rate, conserving the size-distribution, while the kinetic energy is kept constant using a re-scaling thermostat procedure [82].

\subsubsection{Discrete Element Method}

When spherical particles are modelled as soft, they can "overlap". Depending on the material model of the particles, the interaction force can be computed. The spring-dashpot model is the simplest example, where there is a repulsive force proportional to the overlap, and a dissipative force proportional to the relative velocity [83,84]. For a given contact law, the resulting Newton's equations of motion are integrated, usually with a fixed time-step, and the temporal evolution of the system is obtained, for details see Refs. $[85,86]$.

The author of the thesis wrote his own discrete element method code, using the $\mathrm{C}++$ programming language. This code implements several different data structures for efficient contact detection in polydisperse systems, which are discussed in Refs. [87-91] and in Chapter 2. For an open-source code for soft particle simulations that is based strongly on this code ( $\mathrm{C}++$ classes), we refer the reader to the MercuryDPM code [92].

\subsection{Thesis structure}

The thesis is organised into three parts. The first part deals with the numerical problem of contact detection among arbitrarily polydisperse objects (Chapter 2). We present a new efficient algorithm for contact detection whose performance even increases with increasing the degree of polydispersity, unlike most other methods. The performance of the algorithm is theoretically analyzed for various particle size distributions and volume fractions, and recommendations are given concerning the choice of optimal algorithm parameters. Alternative methods were studied in Ref. [87], but are not addressed further. 
The second part, Chapter 3, focuses on the theoretical prediction of the equation of state, i.e., the relation between the volume fraction and pressure of hard-sphere systems with various particle size distributions. The equilibrium equation of state of a fluid mixture of polydisperse hard spheres is well described by considering only the first three moments of the size distribution function. Consequently, these (thermodynamic) properties of a polydisperse fluid can be reproduced by a well-chosen "equivalent" bidisperse fluid with the same three moments. In this study we ask the question: How many moments are needed to predict the pressure and the jamming density of polydisperse mixtures in compressed non-equilibrium glassy states? We find that five moments suffice to describe the properties of polydisperse mixtures for all densities, including glassy, non-equilibrium states and the maximal jamming density. Hence, polydisperse mixtures can be modelled by a wellchosen "maximally equivalent" tridisperse system. Astonishingly, the volume fraction of rattlers is well matched between maximally equivalent systems, and their behavior is shown to be controlled by the non-rattlers.

In the last part, Chapter 4, we suggest a new way to characterize the microstructure in mono- and polydisperse hard-sphere systems, based on the local correlation of up to four particle positions. Our microstructural analysis was shown to be very useful in distinguishing between gaseous, liquid, partially and fully crystallized, and glassy (random) jammed states. A common microstructural feature is observed in crystalline and glassy jammed states, suggesting the presence of "hidden" two-dimensional order in polydisperse random close packings of three-dimensional spheres.

Finally, the conclusions and outlook close this work in Chapter 5. 



\section{Chapter 2}

\section{Contact detection of arbitrarily polydisperse objects}

\subsection{Algorithm description*}

An efficient algorithm for contact detection among many arbitrarily sized objects is developed. Objects are allocated to cells based on their location and size within a nested hierarchical cell space. The choice of optimal cell sizes and the number of hierarchies for best performance is not trivial in most cases. To overcome this challenge, a novel analytical method to determine the optimal hierarchical cell space for a given object size distribution is presented. With this, a decision can be made between using the classical linked-cell method and the contact detection algorithm presented. For polydisperse systems with size ratios up to 50, we achieved 220 times speed-up compared to the classical Linked-Cell method. For larger size ratios, even better speed-up is expected. The complexity of the algorithm is linear with the number of objects when the optimal hierarchical cell space is chosen. So that the problem of contact detection in polydisperse systems essentially is solved.

\subsubsection{Introduction}

Collision detection is a basic computational problem arising in computer simulations of systems consisting of many discrete objects such as particles or atoms. The particle based modeling methods like the Discrete Element Method (DEM) [85] or Smoothed Particle Hydrodynamics (SPH) [93] play an important role for physics-based simulations in various fields. The performance of the computation relies on several factors, which include the physical

\footnotetext{
*Based on V. Ogarko and S. Luding, "A fast multilevel algorithm for contact detection of arbitrarily polydisperse objects," Comput. Phys. Commun., vol. 183, no. 4, pp. 931-936, 2012 .
} 
model, on the one hand, and the contact detection algorithm used, on the other. The collision detection of short-range pairwise interactions between particles in DEM and SPH coupled to DEM is usually one of the most timeconsuming tasks in calculations [94].

The most commonly used method for contact detection of nearly monosized particles with short-ranged forces is the Linked-Cell method [95, 96]. Due to its simplicity and high performance, it has been utilized since the beginning of particle simulations, and is easily implemented in parallel codes $[97,98]$.

Nevertheless, the Linked-Cell method is unable to efficiently deal with particles of greatly varying sizes [99]. This can effectively be addressed by the use of methods based on hierarchical grids [99-105]. Most of these methods can be assigned to two groups. In the first, the contacts between particles from different hierarchy levels are detected in the coarse grid [100-102,104, 105], while in the second group the detection is done in the fine grid [99]; our method corresponds to the latter group. An extensive review of various approaches to contact detection is given in [106]. The performance difference between them is studied in Refs. [48, 87, 107].

Even though various other methods using hierarchical grid structures have been suggested, we improve upon these methods by (i) reducing the range of the contact search, and (ii) using two freely adjustable parameters: the number of hierarchy levels and the cells' size at each level. An analytical method to select these parameters is also developed. This method (for choosing parameters) is designed to improve the performance of the algorithm and can be used for an arbitrary polydisperse particle size distribution. We confirm our theoretical predictions by means of DEM simulations of homogeneous and isotropic systems of elastic spherical particles, even though the algorithm is not limited to these ideal cases.

The chapter is organized as follows. section 2.1.2 outlines the algorithm. We then present the method how to choose the optimal parameters in section 2.1.3. section 2.1.4 presents the performance results of the numerical simulations. Finally, the results are summarized and discussed, with some conclusions in section 2.1.5.

\subsubsection{Algorithm}

The present algorithm is designed to determine all the pairs in a set of $N$ spherical particles in a $d$-dimensional Euclidean space that overlap. Every particle is characterized by the position of its centre $\vec{x}_{p}$ and its radius $r_{p}$. For differently-sized spheres $r_{\min }$ and $r_{\max }$ denote the minimum and the maximum particle radius, respectively, and $\omega=r_{\max } / r_{\min }$ is the extreme size ratio (size distribution functions used are explained in section 2.1.4). 
The algorithm is made up of two phases. In the first "mapping phase" all the particles are mapped into a hierarchical grid space. In the second "contact detection phase" for every particle in the system the potential contact partners are determined, and the geometrical intersection tests with them are made.

\section{Mapping phase}

The $d$-dimensional hierarchical grid is a set of $L$ regular grids with different cell sizes. Every regular grid is associated with a hierarchy level $h \in[1, L]$, where $L$ is the integer number of hierarchy levels. Each level $h$ has a different cell size $s_{h} \in \mathbb{R}$, where the cells are $d$-dimensional cubes. Grids are ordered with increasing cell size so that $h=1$ corresponds to the grid with smallest cell size, i.e., $s_{h}<s_{h+1}$. For a given number of levels and cell sizes, the hierarchical grid cells are defined by the following spatial mapping, $M$, of points $\vec{x} \in \mathbb{R}^{d}$ to a cell at specified level $h$ :

$$
M:(\vec{x}, h) \mapsto \vec{c}=\left(\left\lfloor x_{1} / s_{h}\right\rfloor, \ldots,\left\lfloor x_{d} / s_{h}\right\rfloor, h\right),
$$

where $\lfloor x\rfloor$ denotes the floor function. ${ }^{\dagger}$ The first $d$ components of a $(d+1)$ dimensional vector $\vec{c}$ represent cell indices (integers), and the last one is the associated level of hierarchy. The latter is limited whereas the former are not.

It must be noted that the cell size of each level can be set independently, in contrast to contact detection methods which use a tree structure for partitioning the domain $[48,101,108]$, where the cell sizes are taken as double the size of the previous lower level of hierarchy, hence $s_{h+1}=2 s_{h}$. The flexibility of independent $s_{h}$ allows one to select the optimal cell sizes, according to the particle size distribution, to improve the performance of the simulations. How to do this is explained in section 2.1.3.

Using the mapping $M$, every particle $p$ can be mapped to its cell:

$$
\vec{c}_{p}=M\left(\vec{x}_{p}, h(p)\right),
$$

where $h(p)$ is the level of insertion to which particle $p$ is mapped to. The level of insertion $h(p)$ is the lowest level where the cell is big enough to contain the particle $p$ :

$$
h(p)=\left\{\min _{1 \leq h \leq L} h: s_{h} \geq 2 r_{p}\right\} .
$$

In this way the diameter of particle $p$ is smaller or equal to the cell size in the level of insertion and therefore the classical Linked-Cell method [96]

\footnotetext{
${ }^{\dagger}$ The largest integer not greater than $x$.
} 


\section{Contact detection of arbitrarily polydisperse objects}

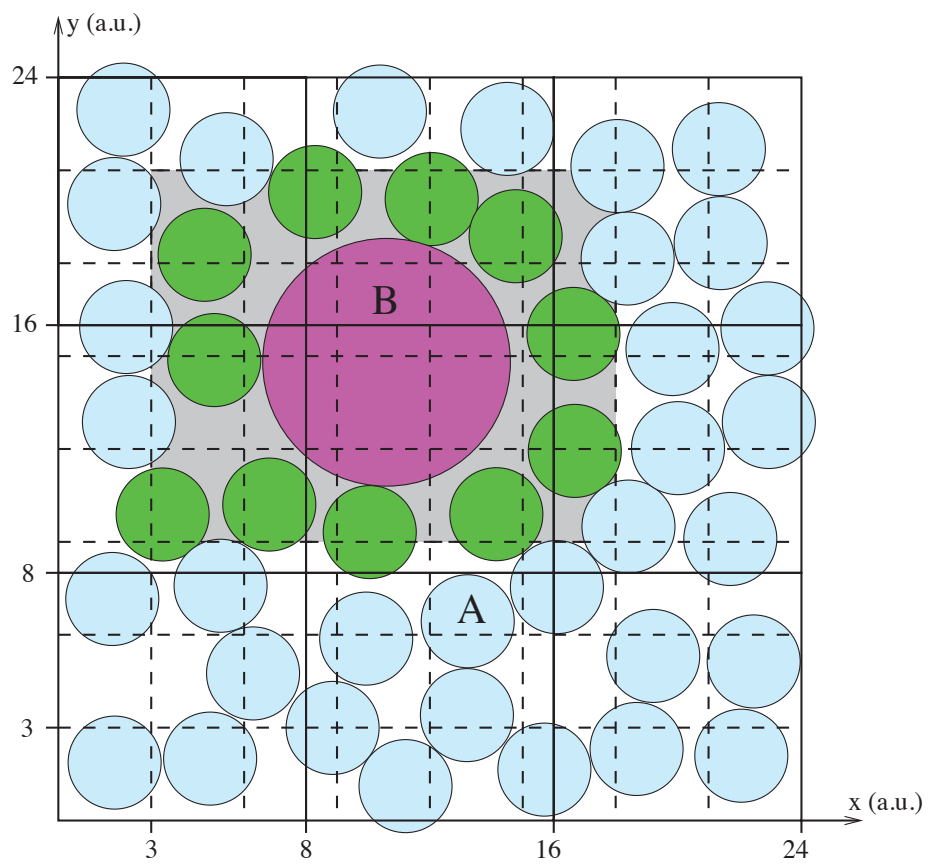

Figure 2.1: A 2-dimensional two-level grid for the special case of a bi-disperse system with cell sizes $s_{1}=2 r_{\min }=3$ (a.u.), and $s_{2}=2 r_{\max }=8$ (a.u.). The first level grid is plotted with dashed lines while the second level is plotted with solid lines. The radius of the particle $\mathrm{B}$ is $r_{\mathrm{B}}=4$ (a.u.) and its position is $\vec{x}_{\mathrm{B}}=(10.3,14.4)$. Therefore, according to Eqs. (2.10) and (2.11), particle $\mathrm{B}$ is mapped to the second level to the cell $\vec{c}_{\mathrm{B}}=(1,1,2)$. Correspondingly, particle A is mapped to the cell $\vec{c}_{\mathrm{A}}=(4,2,1)$. The cells where the cross-level search for particle B has to be performed from $(1,3,1)$ to $(5,6,1)$ are marked in grey, and the small particles which are located in those cells are dark (green). Note, that in the method of Iwai et al [99] the search region starts at cell $(1,2,1)$, i.e., one more layer of cells (which also includes particle A).

can be used to detect the contacts among particles within the same level of hierarchy.

Figure 2.7 illustrates a 2-dimensional two-level grid for the special case of a bi-disperse system with $r_{\min }=3 / 2$, size ratio $\omega=8 / 3$, and cell sizes $s_{1}=3$, and $s_{2}=8$. Since the system contains particles of only two different sizes, two hierarchy levels are sufficient here.

\section{Contact detection phase}

The contact detection is split into two steps, and the search is done by looping over all particles $p$ and performing the first and second steps consecutively for each $p$. The first step is the contact search at the level of insertion of $p$, 


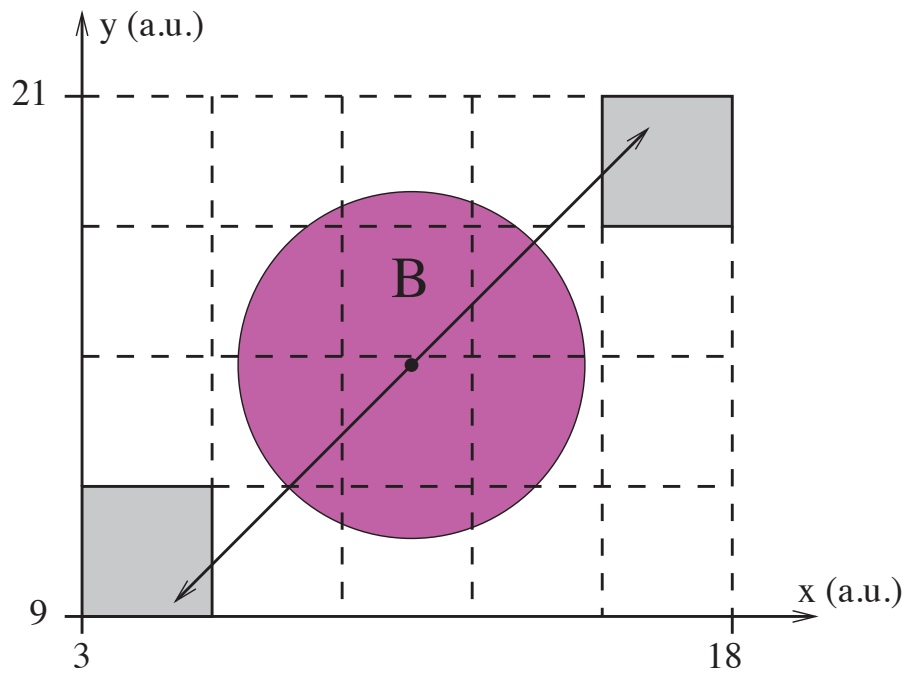

Figure 2.2: The grey colored cells correspond to cells $\vec{c}^{\text {start }}$ (left bottom) and $\vec{c}^{\text {end }}$ (right top) for particle B from the hierarchical grid shown in Fig. (2.7). The two diagonal vectors with length $\alpha$, directed to these cells from the center of particle $\mathrm{B}$, are the vectors $\vec{x}_{c}^{-}$and $\vec{x}_{c}^{+}$respectively.

$h(p)$, using the classical Linked-Cell method [96]. The search is done in the cell where $p$ is mapped to, i.e., $\vec{c}_{p}$, and in its neighbour (surrounding) cells. Only half of the surrounding cells are searched, to avoid testing the same particle pair twice.

The second step is the cross-level search. For a given particle $p$, one searches for potential contacts only at levels $h$ lower than the level of insertion: $1 \leq h<h(p)$. This implies that the particle $p$ will be checked only against the smaller ones, thus avoiding double checks for the same pair of particles. The cross-level search for particle $p$ (located at $h(p)$ ) with level $h$ is detailed here:

1. Define the cells $\vec{c}^{\text {start }}$ and $\vec{c}^{\text {end }}$ at level $h$ as

$$
\vec{c}^{\text {start }}:=M\left(\vec{x}_{c}^{-}, h\right), \text { and } \vec{c}^{\text {end }}:=M\left(\vec{x}_{c}^{+}, h\right),
$$

where a search box (cube in 3D) is defined by $\vec{x}_{c}^{ \pm}=\vec{x}_{p} \pm \alpha \sum_{i=1}^{d} \mathbf{e}_{i}$, with $\alpha=r_{p}+0.5 s_{h}$ and $\mathbf{e}_{i}$ is the standard basis for $\mathbb{R}^{d}$. Any particle $q$ from level $h$, i.e., $h(q)=h$, with center $\vec{x}_{q}$ outside this box can not be in contact with $p$, since the diameter of the largest particle at this level can not exceed $s_{h}$. In Fig. 2.2 the grey colored cells correspond to the cells $\vec{c}^{\text {start }}$ (left bottom) and $\vec{c}^{\text {end }}$ (right top) for particle B from the situation shown in Fig. 2.7. 


\section{Contact detection of arbitrarily polydisperse objects}

2. The search for potential contacts is performed in every cell $\vec{c}=\left(c_{1}, \ldots, c_{d}, h\right)$ for which

$$
c_{i}^{\text {start }} \leq c_{i} \leq c_{i}^{\text {end }} \text { for all } i \in[1, d] \text {, and } c_{d+1}=h<h(p),
$$

where $c_{i}$ denotes the $i$-th component of vector $\vec{c}$. In other words, each particle which was mapped to one of these neighbour cells is tested for contact with particle $p$. In Fig. 2.7, the level $h=1$ cells where that search has to be performed (for particle B) are marked in grey.

To test two particles for contacts, first, the axis-aligned bounding boxes (AABB) of the particles [109] are tested for overlap. Then, for every particle pair which passed this test, the exact geometrical intersection test is applied. ${ }^{\ddagger}$ Since the overlap test for AABBs is computationally cheaper than for spheres, performing such test first usually increases the performance.

\section{Summary}

The two steps of the algorithm, mapping and contact detection were designed for spherical particles. However, other shapes can also be accommodated using bounding spheres; for an overview of methods to compute a bounding sphere see Ref. [101]. Nevertheless, this can affect the performance when particles are rather elongated.

Parallelization of the algorithm and implementation of periodic boundary conditions are straightforward and can be done in almost the same way as in the Linked-Cell method $[110,111]$. Finally, to reduce the memory usage related to storing the cells, we use the hash table approach $[99,101,103]$. This means that the hierarchical grid is not stored explicitly, instead a hash function is used to map only occupied grid cells into a finite 1D hash table.

For bi-disperse particle systems with wide size distributions, for example, $\omega \geq 10$, the use of a two-level grid can lead to a significant improvement as compared to the Linked-Cell method, as we show below in section 2.1.4. Nevertheless, we are interested in finding the optimal grid parameters ( $L$ and $s_{h}$ ) for arbitrarily polydisperse particle systems, which will lead to the best performance. In the next section we present a method how to achieve this.

\subsubsection{Selection of the optimal grid parameters}

The algorithm from the last section is applicable to arbitrary systems (inhomogeneous), whereas for the following analysis, we restrict ourselves to almost homogeneous situations. This does not harm / affect the algorithm, only the performance might be sub-optimal.

${ }^{\ddagger}$ Particles $p$ and $q$ collide only if $\left\|\vec{x}_{p}-\vec{x}_{q}\right\|<r_{p}+r_{q}$, where $\|\cdot\|$ is Euclidean norm. 


\section{Bi-disperse systems}

For bi-disperse particle systems the cell sizes of the two-level grid can be easily selected as the two diameters of each particle species. For some situations this may be not as efficient as the use of the single-level Linked-Cell method. In section 2.1.4 we show some performance results for bi-disperse size distributions.

\section{Polydisperse systems}

In polydisperse systems all the particles' sizes are different. This is the case when a particle sample is drawn from a continuous particle size distribution (PSD), for example using a systematic sampling approach [112,113]. It guarantees an evenly spread sample, i.e., always includes some of the possibly rare largest particles in a sample, see Fig. 2.3. For such systems the parameters of the algorithm (the number of levels $L$ and cell sizes $s_{h}$ ) can be chosen in different ways. The performance of the hierarchical grid algorithm then strongly depends on the selected parameters.

For example, consider different numbers of hierarchy levels $L$. The fewer levels are used, the larger the number of particles per cell. This implies that a larger amount of particles pairs will be found in the contact search, affecting the CPU time dramatically when the system has a large number of small particles. Increasing the number of hierarchy levels will decrease the number of particle pairs found in the contact search. However, this will increase the number of cross-level tests, and hence the number of cells which have to be accessed, negatively affecting the CPU time. To obtain the optimal performance it is important to balance the number of particle pairs found with the number of cells to be checked.

Assume the following hypothesis holds:

Hypothesis. Let $m_{h}$ be the average number of particles per cell at level $h$, that is, $m_{h}=N_{h} / N_{h}^{c}$, where $N_{h}$ is the number of particles at level $h$, and $N_{h}^{c}$ is the number of cells at this level. Then the optimal distribution of particles by levels satisfies the following condition:

$$
m:=m_{i}=m_{j} \text {, for all } i, j \in[1, L] .
$$

The detailed discussion of this is given in the next section.

If the particles are mapped to the levels, such that Eq. (2.6) is approximated, it can be shown that the CPU time spent for contact detection, $T_{C D}$, scales as

$$
T_{C D} \sim N L(m+K)
$$


where $K$ is a constant corresponding to the "overhead" of the algorithm, i.e., the time spent to access cells to be tested, and $m=m(L, \mathrm{PSD})$ is the number of particles per cell. To compute $m$ for a given $L$ and for the particle system at hand, one needs to choose cell sizes $s_{h}$ so that Eq. (2.6) is approximately satisfied. How to do this is explained in 2.1.5. Derivation of Eq. (2.7) is beyond the scope of this paper. Here, the comparison of the performance results with the prediction of Eq. (2.7) will be shown.

As obtained from numerical experiments in section 2.1.4, the value of $K$ varies in a narrow range $[0.2, \ldots, 0.45]$, depending on the size distribution, and is set to 0.3 with sufficient accuracy. For more details, see Ref. [88].

We propose to use as the optimal number of levels (ONL) the one that minimizes the right hand side of Eq. (2.7). It must be noted that in general the ONL is relatively small. For example, in the system below with $\omega=50$ with uniform volume distribution, see Fig. 2.4(d), ONL $=7$.

\subsubsection{Numerical experiments}

The aim of this section is to test the presented algorithm in physically realistic, dilute to dense polydisperse gas- and fluid-like systems. For these we verify experimentally the analytical prediction from Eq. (2.7). More specifically, we use homogeneous and isotropic disordered systems of colliding elastic spherical particles in a unit cubical box with hard walls. The motion of particles is governed by Newton's second law with a linear elastic contact force during overlap. For simplicity, every particle undergoes only translational motion (without rotation) and gravity is set to zero.

\section{Particle size distributions}

The following types of particle size distributions are used: (i) monodisperse, i.e., all sizes are equal; (ii) bi-disperse (Bi), i.e., two different sizes, where the volume of all small particles and the volume of all big particles are equal, which is also used in [114]; (iii) uniform size distribution (US), i.e., the distribution of radii of the particles is constant; (iv) uniform volume distribution (UV), i.e., the distribution of the volumes of the particles is constant. Figure 2.3 shows the relative frequencies of the particle radii appearing in some realizations of the aforementioned particle size distributions, as displayed as snapshots in Fig. 3.5.

We believe that these types of distributions cover the most important cases to check the efficiency of the presented algorithm. Systems with monodisperse size distributions are widely used since kinetic theory predicts their physical behaviour $[2,115,116]$, and it is the natural benchmark against which to compare. Bi-disperse size distributions are often used for theo- 


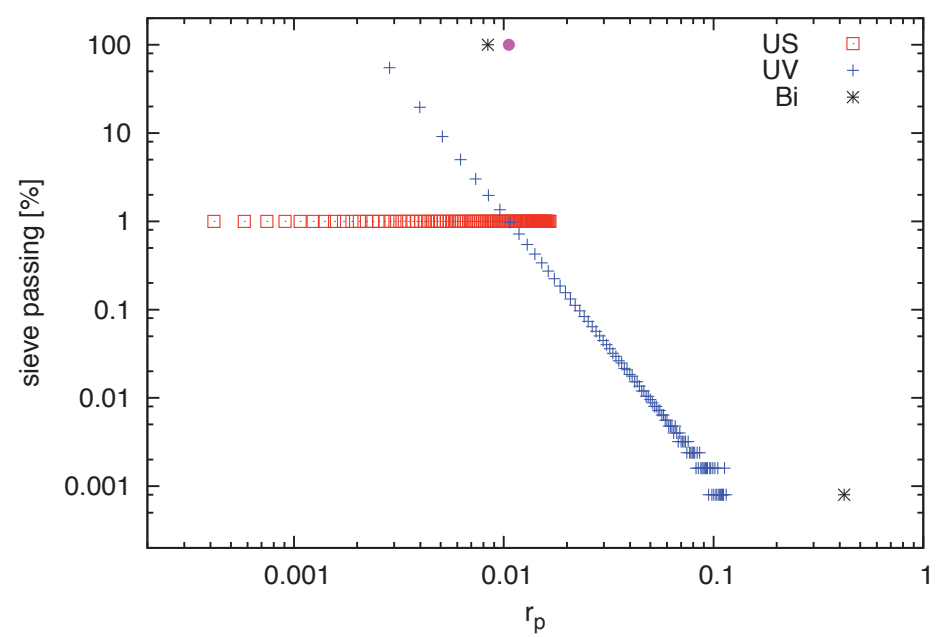

Figure 2.3: The relative frequencies of radii of particles of size distributions described in section 2.1.4 with $N=125001, \nu=0.62$ and $\omega=50$, see Figs. 2.4(c) and 2.4(d) for snapshots of these systems. The filled circle corresponds to the monodisperse system $(\omega=1)$ with the same $\nu$. The number of equidistant bins used is 100 .

retical models $[114,117,118]$, and can often be a good approximation to physically realistic size distributions. Uniform size and uniform volume distributions are selected in order to check the speed-up of the multilevel grid for polydisperse systems with relatively few small particles (uniform size), or rather many small particles (uniform volume). The uniform volume distribution approximates the experimentally obtained size distribution of a concrete mixture [113].

Figure 3.5 shows the particle systems with different particle size distributions for $N=125001$ and volume fraction (the ratio between the volume of the particles and the volume of the system) $\nu=0.62$. Note, that particles of the monodisperse case are ordered (near to walls), since the volume fraction is above $0.55[116,119,120]$.

\section{Experimental setup}

The systems are prepared in two stages. Starting from a random uniform distribution of points in a cubical box, the radius of the particles grows linearly with time. We use non-overlapping spheres [81], with an EventDriven code [67], whose growing rate conforms to and conserves a prescribed size distribution. Initial velocities are set randomly in order to keep the system dynamic and random, for details see Ref. [67]. When the target 


\section{Contact detection of arbitrarily polydisperse objects}

volume fraction is reached, the growth process is stopped.

With the final configuration from the first step, the simulation switches to the relaxation stage with "soft" particles, i.e., particles move according to interparticle forces [85]. The linear elastic normal contact force model is used [84], which leads to a certain contact duration. The integration time step is computed according to the smallest contact duration [84,121]. At the beginning of this stage the velocities of the particles are scaled in a way that a collision between two of the smallest particles would reach an average maximum overlap of one percent of their radius. We let the simulations run for a few collisions per particle for equilibration before making the measurement of the performance, in order to have contacts (overlaps) between particles in the system.

\section{Experimental results}

To verify the prediction of Eq. (2.7) we perform two series of experiments, one for varying number of hierarchy levels, and one for different numbers of particles. In the first series, we want to confirm that the multiplier next to $N$ is $L(m+K)$. For this, using a fixed $N$, we calculate the value of $m$ for each $L \in[1,50]$ utilizing the method given in 2.1.5, and measure the total CPU time of simulations where the hierarchical grid is used with $L$ levels, and the cell sizes $s_{h}$ are computed in accordance with Eq. (2.6). To present the total CPU time, we use the slowdown factor SF, that is the total $\mathrm{CPU}$ time divided by the smallest $\mathrm{CPU}$ time for a given system. In Fig. 2.5 the results of this experiment are shown for systems with uniform size (US) and uniform volume (UV) distributions with $N=125001, \nu=0.62$ and $\omega=50$. The analytical prediction (2.7) is also plotted with $K=0.3$, scaled in such a way that $\mathrm{SF}=1$ corresponds to the minimum of the right hand side of Eq. (2.7). Note that even though the prediction (2.7) is for CPU time spent only for contact detection, $T_{C D}$, the total CPU time for fixed $N$ also scales as $T_{C D}$. This is because the CPU time spent in the force calculation and integration does not depend on the grid parameters used. From the experimental results shown in Fig. 2.5 it can be seen that: (i) for the system with uniform size distribution the optimal number of levels is $L=3$, and the speed-up compared to the Linked-Cell method $(L=1)$ is about $30 \%$, so it does not present a major advantage; (ii) in the case of uniform volume distribution the fastest CPU time is achieved using $L=8$, and the speed-up over the Linked-Cell method $(L=1)$ is of about 220 times. This brings us to the conclusion that for particle systems with relatively few small particles, the use of hierarchical grid algorithm is not essential; however, for the systems with rather many small particles the hierarchical grid algorithm is highly advantageous. Furthermore, it can be seen that the 


\section{Algorithm description}

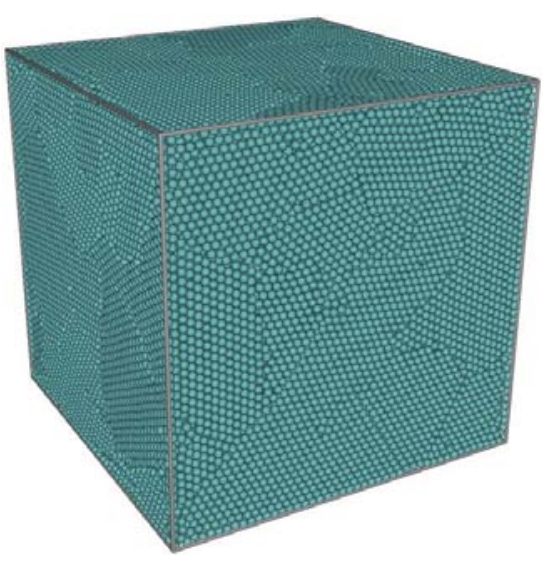

(a)

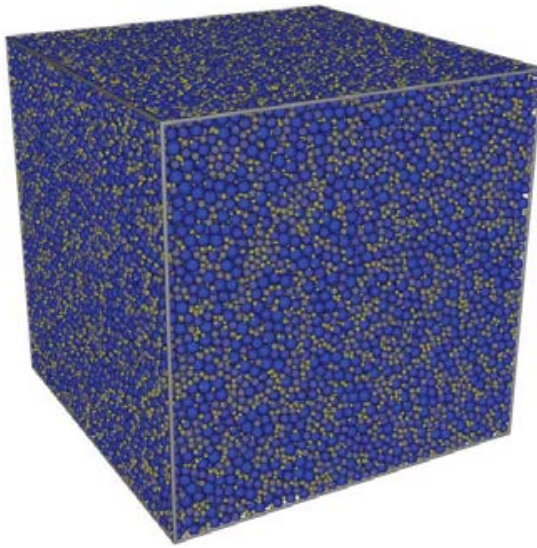

(c)

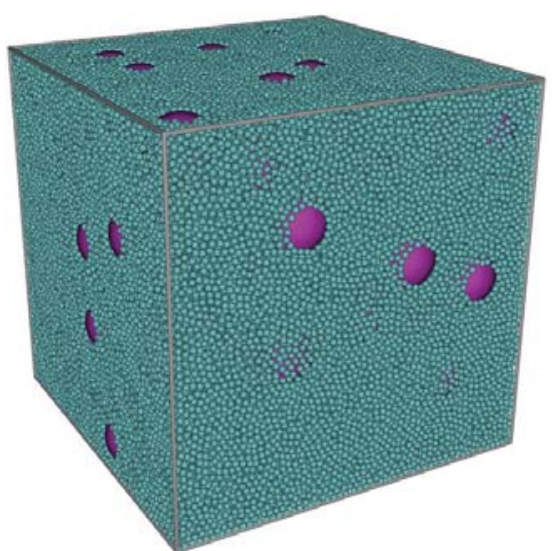

(b)

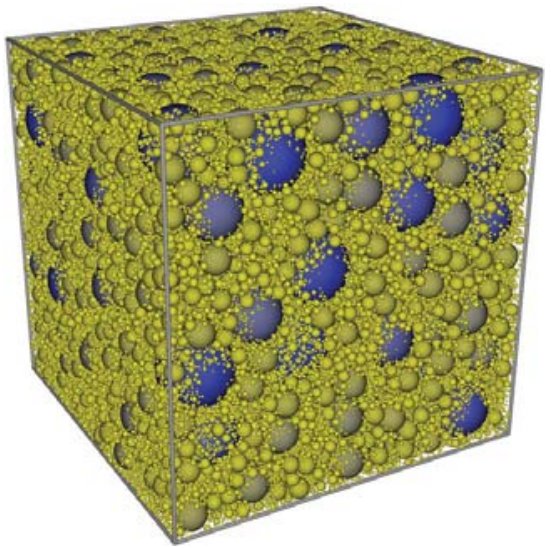

(d)

Figure 2.4: Particle systems with $N=125001$ and $\nu=0.62$ with (a) monodisperse size distribution, (b) bi-disperse size distribution, $\omega=10$, where the volume of all small particles is almost $( \pm 2 \%)$ equal to the volume of all big particles, (c) uniform size distribution, $\omega=50$, and (d) uniform volume distribution, $\omega=50$. Colour is by relative size for the cases (c) and (d).

analytical prediction (2.7) is in very good agreement with the experimental results. We have also performed the same type of experiment for systems with different volume fractions, namely, $\nu=0.1$ and $\nu=0.4$, and in all these cases the prediction matches very well with the experimental results [88].

In the second series of experiments we confirm that the CPU time spent for contact detection scales linearly with the number of particles. Figure 2.6 shows the total CPU time relative to the monodisperse case, $T^{r e l}$. Polydisperse systems with uniform volume distribution are considered, since it was shown above that for uniform size distributions the use of hierarchical 
grid algorithm is not essential. The results for bi-disperse systems are also shown, with $L=2$ and cell sizes equal to the diameters of the each kind of particles. It can be seen that the CPU time scales as $O(N)$, since $T_{\text {rel }}$ is approximately constant $( \pm 10 \%)$ for each type of system, and because the Linked-Cell method for monodisperse particles is $O(N)$. Secondly, the CPU time is close to the monodisperse case $\left(T^{r e l}=1\right)$, and the largest difference is about $50 \%$ in the polydisperse case with $\omega=10$. Furthermore, the CPU time decreases with increasing $\omega$ at constant volume fraction. This is since the mean free path of the system decreases with $\omega$, which determines the number of contact neighbors found, hence the computation time. It must be noted that for large $\omega$ we cannot create representative samples with few particles, so we cannot compare all the systems for every $N$ used in the monodisperse case.

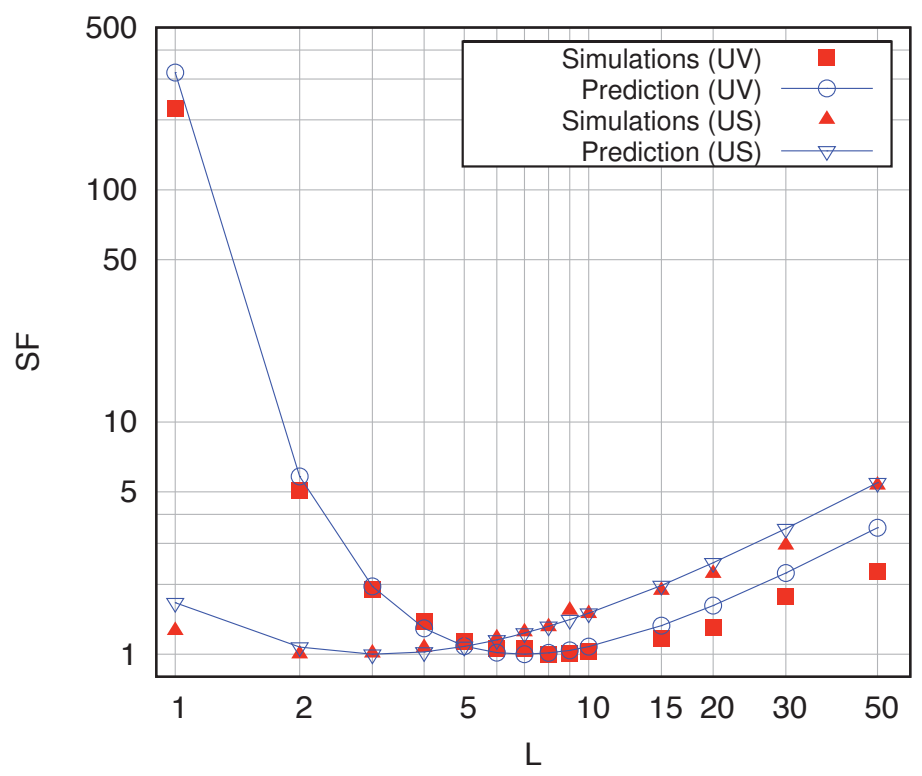

Figure 2.5: Slowdown factor for different numbers of levels for systems with $N=125001, \nu=0.62$ and $\omega=50$ with uniform volume (UV) and uniform size (US) distributions. The prediction of Eq. (2.7) is used with $K=0.3$ for plotting solid lines. Note that the data can be obtained only for integer values of $L$.

\subsubsection{Summary and Conclusions}

A multilevel algorithm for contact detection in systems with arbitrarily polydisperse objects' sizes was developed. DEM simulations were carried out to 


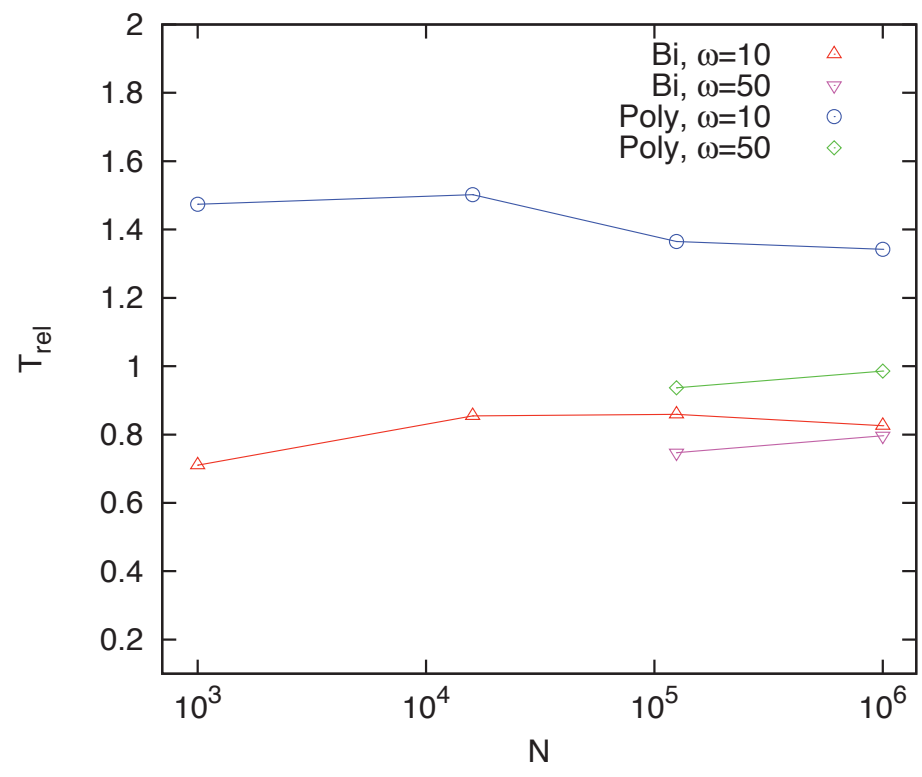

Figure 2.6: The total CPU time scaled by the total CPU time of a monodisperse system with the same number of particles and volume fraction, simulated for the same number of iterations. We show results for bi-disperse (Bi) and polydisperse uniform volume (Poly) size distributions. Extremely wide size distributions cannot be properly realized for too small $N$.

assess the performance of the algorithm, which contains as adjustable parameters the number of hierarchy levels and the cell sizes at each level. A method to find the optimal parameters for an arbitrary polydisperse size distribution of objects was suggested and confirmed by numerical experiments. With the optimal parameters our simulations can run orders of magnitude faster than when using the (single level) Linked-Cell method. With this algorithm we are able to simulate objects which have large size ratios with almost the same computational time as in the monodisperse case. With parameters selected using our method the performance of the algorithm scales linearly with the number of particles for any width of the size distribution tested. Our work opens the door to simulate realistic polydisperse systems, making possible a complete new range of simulations.

Having solved the problem of fast contact detection for largely different particle sizes, the challenge due to strongly different time-scales (contact durations) of small and large particles remains: the smallest particles are critical, since they are the fastest to react / accelerate given the same force.

In a future study, we will build on the observation, see Fig. 2.3, that the smallest particle size in the US case is smaller than in the UV case, which 
is smaller than the bi-disperse case [27]. We will propose equivalent bidisperse systems that have the same equation of state as polydisperse ones, but have a narrower size-distributions, including larger minimal particles. These equivalent bi-disperse systems involve a larger minimal time-scale and are thus (at least computationally) advantageous.

\section{Appendix}

\section{Calculation of $m(L$, PSD $)$}

Assume that particles are sorted by size in increasing order, so that $r_{i}>r_{j}$ for $i>j$. We iteratively increase the value of $m$ by $\delta m \ll 1$ starting from zero. Then for every $m$ we distribute particles by levels as following: Starting with the first level, i.e., $h=1$, and from the smallest particle, i.e., $i=1$, we allocate the $i$-th particle at level $h$. We increase the level index $h$ if for some $i$ the number of particles per cell at current level exceeds $m$ :

$$
\frac{N_{h}(i)}{N_{h}^{c}(i)}>m,
$$

where $N_{h}(i)$ is the number of particles already allocated at level $h$ (including the particle $i$ ) and $N_{h}^{c}(i)$ is the number of cells at level $h$. The number of cells $N_{h}^{c}(i)$ is calculated from the size of particle $i$, e.g., for a $d$-dimensional cube with size of $S, N_{h}^{c}=\left[S /\left(2 r_{i}\right)\right]^{d}$. This process is finished when all particles are allocated. Therefore, we obtain the number of levels for a given $m$. Different $m$ can lead to the same $L$ value. In this case, we select $m(L, P S D)$ with the minimum value, so the last level contains more particles. For numerical experiments in section 2.1.4 the use of $\delta m=10^{-3}$ for the system with $\omega=50$ with uniform volume distribution and $L=7$ led to about $\pm 2 \%$ variation of the number of particles per cell at different levels. 


\section{$2.2 \quad$ Performance analysis ${ }^{\S}$}

The objective of this section is to find the optimum cell-sizes and the number of hierarchy levels for contact detection algorithms based on a versatile hierarchical grid data structure, for polydisperse particle systems with arbitrary size distribution of radii. These algorithms perform as fast as $O(N)$ for $N$ particles, but the prefactor can be as large as $N$ for a given system, depending on the algorithm parameters chosen, making a recipe for choosing these parameters necessary. We estimate theoretically the calculation time for two distinct algorithms for particle systems with various packing fractions, where the sizes of the particles are modelled by an arbitrary probability density function.

We suggest several methods for choosing the number of hierarchy levels and the respective cell-sizes, based on truncated power-law radii distributions with different exponents and widths. The theoretical estimations are then compared with simulation results for particle systems, with up to one million particles.

The proposed recipe for selecting the optimal hierarchical grid parameters allows to find contacts in arbitrarily polydisperse particle systems as fast as the commonly-used Linked-Cell method in purely monodisperse particle systems, i.e., extra work is avoided in presence of polydispersity. Furthermore, the contact detection time per particle decreases with increasing polydispersity or decreasing particle packing fraction.

\subsubsection{Introduction}

Collision detection is a basic computational problem arising in systems that involve spatial interactions among many objects such as particles, granules or atoms in many diverse fields such as robotics, computer graphics, physical simulations, cloth modelling, computational surgery, crowd simulations, etc; which all have rather short-ranged interactions in common. The particle systems modelling techniques like the Discrete Element Method, (Event-Driven) Molecular Dynamics, Monte-Carlo simulations and Smoothed Particle Hydrodynamics, to name a few, play an important role for physically based simulations of powders, granular materials, fluids, colloids, polymers, liquid crystals, proteins and other materials. The performance of the computation relies on several factors, which include the physical model, on the one hand, and the contact detection algorithm used, on the other. The contact

\footnotetext{
§Based on D. Krijgsman, V. Ogarko, and S. Luding, "Optimal parameters for a hierarchical grid data structure for contact detection in arbitrarily polydisperse particle systems." Accepted in Comp. Part. Mech., 2014. The contribution of the first two authors to this work was equal in relation to the performance analysis and writing the manuscript.
} 


\section{Contact detection of arbitrarily polydisperse objects}

detection of pairwise interactions between particles can be one of the most time-consuming tasks in calculations when no suitable contact detection algorithm used. Because the number of objects treated in simulations is often large, contact detection can become a computational bottleneck. For this reason, the development of efficient contact detection algorithms is crucial to the overall performance of simulations.

With the straightforward "all-to-all" approach each pair of particles is checked for collision. This requires $O\left(N^{2}\right)$ collision checks for $N$ particles, which is computationally prohibitively expensive. More efficient contact detection methods use a two-phase approach to reduce the computational costs [122]. The problem of contact detection is then split into two phases: a broad phase and a narrow phase. The broad phase determines pairs of objects that might possibly collide. It is frequently done by dividing space into regions and testing if objects are close to each other in space. Because objects can only intersect or interact if they occupy the same region of space, the number of pairwise tests can be reduced to $O(N)$. The pairs that "survive" the broad phase test are passed to the narrow phase, which uses specialized techniques to test each candidate pair for a real contact [123-125]. The latter is trivial for spherical particles, where one has to compare the distance between particle centers with the sum of particle radii, and can be very costly for particles of arbitrary shape. For example, if there are $S$ surface points per particle, a naive scheme may take order $O\left(S^{2}\right)$ operations. More sophisticated schemes, such as the discrete function representation scheme, require on average $O\left(S^{1 / 2}\right)$ operations [123]. Since the broad phase basically acts as a filter for the narrow phase, choices for the two algorithms can usually be made independently.

We distinguish three types of broad phase contact detection methods/data structures: (i) based on coordinate sorting (or spatial sorting), e.g., sweep and prune, (ii) based on Delaunay triangulation, and (iii) based on spatial subdivision, e.g., (hierarchical) grids (or cell-based methods) and trees (e.g., Octrees in $3 D$ and Quadtrees in $2 D$ ). Below we briefly describe the above methods and their advantages and weaknesses, while for the detailed analysis see Refs. $[48,87,101,106,107,122,126]$ and references therein.

Contact detection algorithms based on coordinate sorting imply maintaining particles in a sorted structure along each axis $[127,128]$. These methods are not sensitive to the particle sizes (i.e., radii for spherical particles) and consume $O(N)$ memory; but they require sorting, which can range in effort from $O(N)$ to $O\left(N^{2}\right)$, depending on the sorting method used, volatility of the sorting lists over time and spatial distribution of objects.

The Delauney triangulation data structure consumes $O(N)$ memory and it is not sensitive to the particle sizes when weighted triangulation is used 
[129]. However, it has the disadvantage that building (or re-building) the tree has a high computational cost, especially for moving particles. The use of flipping algorithms for maintaining and only incrementally updating the triangulation allows decreasing the overhead of re-building the triangulation [87], but unfortunately in three-dimensional system flipping can get "stuck" [130]. Furthermore, its parallelization and maintaining of periodic boundary conditions (which are frequently used in particle simulations) is complicated.

The tree data structure for contact detection does not allow to choose cell size at every level of hierarchy independently, therefore, leaving no room for its optimization for various distribution of particle sizes $[108,131,132]$. Moreover, accessing neighbour sub-cubes in the tree is not straightforward since they can be nodes of different tree branches; no more details are given here since this method is not used any further.

The single-level grid-based contact-detection methods, like for example the Linked-Cell method [95, 96, 133], are straightforward, widely used and perform well for similarly sized objects. The problem of such methods is their inability to efficiently deal with particles of greatly varying sizes [99]. If the particles within the system are polydisperse, the cell size of a grid would have to conform to the largest particle size. Then many small particles may occupy the same cell which increases the number of pairwise checks, and therefore affects the computational performance a lot.

This size problem can effectively be addressed by the use of multi-level hierarchical grids $[89,99,101-105,122]$. Particles are positioned at different levels (according to their size) and collision checks are performed in two steps: (i) within the level of insertion (which is usually performed in the same way as in the Linked-Cell method), and (ii) cross-level checks. The cell size at each hierarchy level can be selected independently, therefore one can adapt grid cells according to a given particle size distribution. Several algorithms based on the hierarchical grid data structures were employed, which differ in the way in which the above two steps are implemented.

The hierarchical grid data structure performs $O(N)$ for arbitrary polydisperse system and uses $O(N)$ memory. This data structure is robust, can be easily parallelized, allows straightforward handling of periodic boundary conditions and can easily deal with unbounded systems. Moreover, it provides $O(1)$ access to the particle data and to all particle nearest neighbours, and, more importantly, allows for $O(1)$ particle insertion and removal from the system, which is often needed in modelling of dynamical systems, like for example hopper or granular flows. Finally, it provides a natural multi-scale framework as particles from different hierarchy levels usually have different physical properties besides their size. For example, small particles are often fast (i.e., have higher velocity/energy) and big ones are slow, so they can 


\section{Contact detection of arbitrarily polydisperse objects}

have different time scales at different hierarchy levels. These are the reasons why we chose the hierarchical grid as our primary data structure for contact detection and analyse how to optimize it for fastest contact detection in widely polydisperse particle mixtures.

The hierarchical grid data structure has many parameters to configure, i.e., an arbitrary choice of the number of hierarchy levels plus an arbitrary choice of the cell size at every level of hierarchy (the cell sizes are, as convention, increasing with increasing level of hierarchy). The choice of parameters affects the number of contact checks and the overhead of the algorithm, i.e., the number of times the cells are accessed. Due to the many parameters involved, finding the optimal ones, i.e., those which minimize an average number of calculations, $T$, is a non-trivial problem. This involves multidimensional optimization where the optimum dimension is unknown. We are not aware of any study where this question was fully addressed, except for the study by Ogarko et al. [89] in which the authors tried to address this problem by providing a hypothesis on the optimal choice of the hierarchical grid parameters, and then comparing their theoretical predictions with the simulation results.

In this study we theoretically analyse the performance complexity of the hierarchical grid data structure for contact detection in polydisperse particulate systems. We provide detailed analysis on the average number of calculations, $T$, for two distinctive algorithms based on the hierarchical grid data structure as applied to polydisperse systems of spherical particles with a power-law distribution of radii, for various power-law exponents, and for various particle volume fractions. We compare several ways (methods) of choosing the hierarchical grid parameters (i.e., the number of levels and the cell sizes at each level) and present the optimal parameter choice. We provide instructions on which hierarchical grid contact detection algorithm should be used and how to choose the optimal parameters for a given arbitrary distribution of particle radii. Finally, we compare our theoretical predictions with simulation results for realistic particle systems generated using a Molecular Dynamics code [134].

In the next section we outline the two different algorithms based on the hierarchical grid data structure that are used in this study. We then analyse the performance of the described algorithms and derive general estimates for the number of contact checks per particle in section 2.2.3. section 2.2.4 presents the types of particle size distributions considered in this study. In section 2.2.5 we compare several ways of choosing the hierarchical grid parameters and check our analytical estimates by comparing them with real discrete particle simulations, using the MercuryDPM code [92]. Finally, the results are summarized and discussed in section 2.2.6. 


\subsubsection{Algorithm}

The hierarchical grid (HGrid) algorithm is designed to determine all pairs of particles, in a set of $N$ particles in a $d$-dimensional Euclidean space, that overlap or interact. The split between local particle geometry and global neighbour searching is achieved through the use of a bounding volume. This way, the contact detection algorithm is able to treat all particle shapes in the same, simplified way. While any bounding volume can be used, the sphere is chosen for this implementation since it is represented simply by a position of its center $\vec{x}_{p}$ and its radius $r_{p}$, and is rotationally invariant. For differentlysized spheres, $r_{\min }$ and $r_{\max }$ denote the minimum and the maximum particle radius, respectively, and $\omega=r_{\max } / r_{\min }$ is the extreme size ratio.

The algorithm consists of two phases. In the first "mapping phase" all the particles are mapped into a hierarchical grid-space. In the second "contact detection phase" the potential contact partners are determined for every particle in the system. This list of potentially contacting particle pairs is the output of the algorithm. With this list one can perform geometrical intersection tests to check if particles are really in contact, i.e., if they overlap. However, for non-spherical particles, this is beyond the scope of the present algorithm

Requirements for the algorithm are:

- All pairs of particles that are in contact must be in the list of potential contacts, i.e., the algorithm is not allowed to miss any pair.

- The list of pairs of particles must be unique, i.e., no pair of contacts may appear twice in the list.

- The list of pairs of particles should be as small as possible.

- The computational time of the algorithm should be as small as possible, and thus must scale linearly with the number of particles, i.e., $O(N)$.

- The memory consumption of the algorithm must be proportional to the number of particles, i.e., $O(N)$.

\section{Mapping phase}

The $d$-dimensional HGrid is a set of $L$ regular grids with different cell sizes. Every regular grid is associated with a hierarchy level $h \in[1, L]$, where $L$ is the integer number of hierarchy levels. Each level $h$ has a different cell size $s_{h} \in \mathbb{R}$, where the cells are $d$-dimensional cubes. Grids are ordered with increasing cell size so that $h=1$ corresponds to the grid with smallest cell size, i.e., $s_{h}<s_{h+1}$. For a given number of levels and corresponding cell sizes, 


\section{Contact detection of arbitrarily polydisperse objects}

the hierarchical grid-cells are defined by the spatial mapping, $M$, of points $\vec{x} \in \mathbb{R}^{d}$ to a cell at specified level $h$ :

$$
M:(\vec{x}, h) \mapsto \vec{c}=\left(\left\lfloor x_{1} / s_{h}\right\rfloor, \ldots,\left\lfloor x_{d} / s_{h}\right\rfloor, h\right),
$$

where $\lfloor x\rfloor$ denotes the floor function. " The first $d$ components of a $(d+1)$ dimensional vector $\vec{c}$ represent cell indices (integers), and the last one is the associated hierarchy level.

It must be noted that the cell size of each level can be set independently, in contrast to contact detection methods which use a tree structure for partitioning the domain $[101,108,131]$, where the cell sizes are taken as double (or triple) the size of the previous lower level of hierarchy, hence $s_{h+1}=2 s_{h}$ (or $3 s_{h}$ ). The flexibility of independent $s_{h}$ allows one to select the optimal cell sizes, according to the particle size distribution, to improve the performance of the contact detection algorithm.

Using the mapping $M$, every particle $p$ can be mapped to its cell:

$$
\vec{c}_{p}=M\left(\vec{x}_{p}, h(p)\right),
$$

where $h(p)$ is the level of insertion to which particle $p$ is mapped to. The level of insertion $h(p)$ is the lowest level, where the cell is big enough to contain the particle $p$ :

$$
h(p)=\left\{\min _{1 \leq h \leq L} h: 2 r_{p} \leq s_{h}\right\} .
$$

In this way the diameter of particle $p$ is smaller or equal to the cell size at the level of insertion and therefore the classical Linked-Cell method [96] can be used to detect contacts among particles within the same level of hierarchy.

Figure 2.7 illustrates a 2-dimensional two-level grid for the special case of a bi-disperse system with $r_{\text {min }}=3 / 2$, size ratio $\omega=8 / 3$, and cell sizes $s_{1}=3$, and $s_{2}=8$. Since the system contains particles of only two different sizes, two hierarchy levels are sufficient here.

\section{Contact detection phase}

After all particles are mapped to their cells, the contact detection phase is able to calculate all potential contacts. The contact detection is performed by looping over all particles $p$ and searching for possible contacts with particles at the same hierarchy level $h$ and for possible contacts at different hierarchy levels.

Searching for contacts at the same hierarchy level is performed using the

"The largest integer not greater than $x$. 

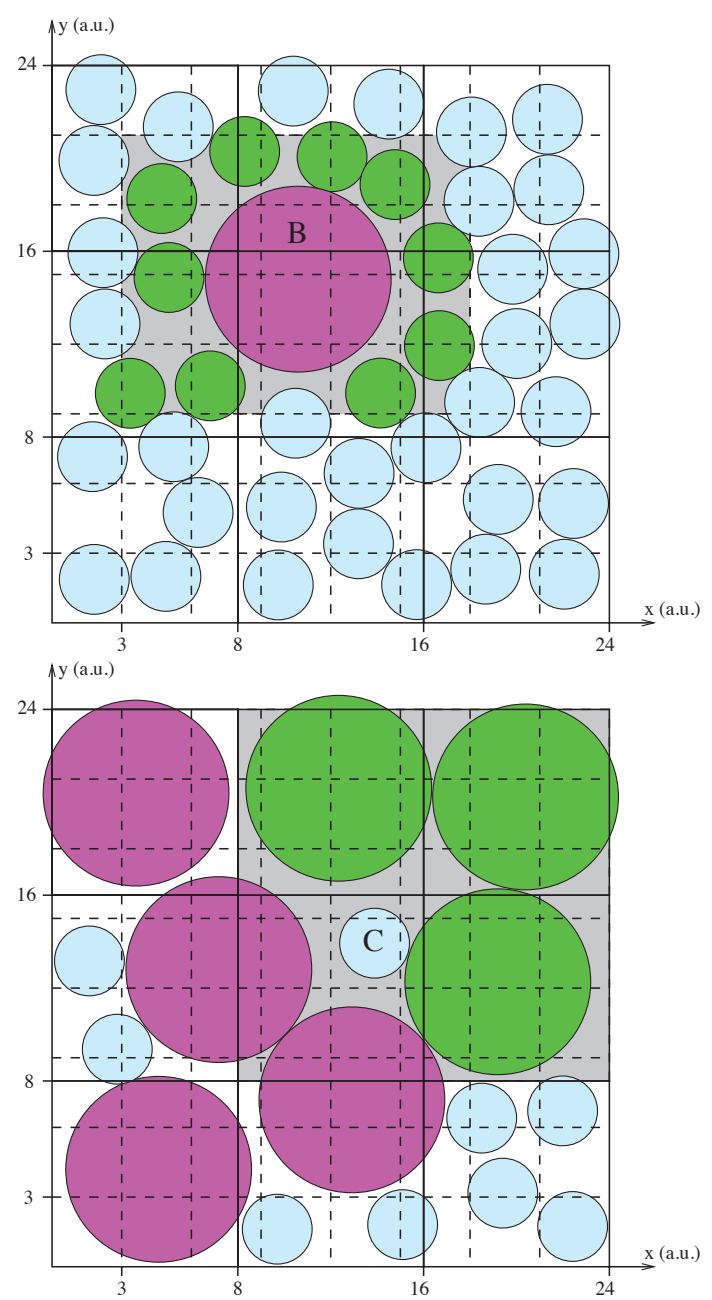

Figure 2.7: A 2-dimensional two-level grid for the special case of a bi-disperse system with cell sizes $s_{1}=2 r_{\min }=3$ (a.u.), and $s_{2}=2 r_{\max }=8$ (a.u.). The first level grid is plotted with dashed lines while the second level is plotted with solid lines. (Top) The Top-Down case: The radius of the particle B is $r_{\mathrm{B}}=4$ (a.u.) and its position is $\vec{x}_{\mathrm{B}}=(10.3,14.4)$. Therefore, according to Eqs. (2.10) and (2.11), particle B is mapped to the second level to the cell $\vec{c}_{\mathrm{B}}=(1,1,2)$. The cross-level cells that have to be checked for possible contacts with particle B range from $(1,3,1)$ to $(5,6,1)$, and are marked in grey. (Bottom) The Bottom-Up case: A particle $\mathrm{C}$ is mapped to the cell $\vec{c}_{\mathrm{C}}=(4,4,1)$. The cross-level cells that have to be checked for possible contacts with particle $\mathrm{C}$ range from $(1,1,2)$ to $(2,2,2)$, and are marked in grey. The particles located in the marked (grey) cells are coloured dark (green).

classical Linked-Cell method [96]. The search is done in the cell where $p$ is 


\section{Contact detection of arbitrarily polydisperse objects}

mapped to, i.e. $\vec{c}_{p}$, and in its neighbouring (surrounding) cells. Only half of the surrounding cells are searched, to avoid testing the same particle pair twice.

Searching for contacts at other hierarchy levels can be performed in two ways. The first one is the Top-Down method, illustrated in Fig. 2.7(top). In this method one searches for potential contacts only at levels $j$ lower than the level of insertion: $1 \leq j<h$. This implies that the particle $p$ will be checked only against smaller particles, thus avoiding double checks for the same pair of particles. The second method, the Bottom-Up method, sketched in Fig. 2.7(bottom), does exactly the opposite. Here potential contacts are only searched for at hierarchy levels $j$ higher than the level of insertion: $h<j \leq L$. This implies that the particle $p$ will be checked only against larger particles, thus avoiding double checks for the same pair of particles.

The details for both methods are actually quite similar. The algorithm to find potential contacts for particle $p$ at hierarchy level $h$ with other particles at hierarchy level $j$ is as follows:

1. Define the cells $\vec{c}^{\text {start }}$ and $\vec{c}^{\text {end }}$ at level $j$ as

$$
\vec{c}^{\text {start }}:=M\left(\vec{x}_{c}^{-}, j\right) \text {, and } \vec{c}^{\text {end }}:=M\left(\vec{x}_{c}^{+}, j\right),
$$

where a search box (cube in 3D) is defined by $\vec{x}_{c}^{ \pm}=\vec{x}_{p} \pm \beta \sum_{i=1}^{d} \mathbf{e}_{i}$, with $\beta=r_{p}+s_{j} / 2$ and $\mathbf{e}_{i}$ is the standard basis for $\mathbb{R}^{d}$. Any particle $q$ from level $j$, with center $\vec{x}_{q}$ outside this box can not be in contact with particle $p$, since the diameter of the largest particle at this level can not exceed $s_{j}$.

2. The search for potential contacts is performed in every cell $\vec{c}=\left(c_{1}, \ldots, c_{d}, j\right)$ for which

$$
c_{i}^{\text {start }} \leq c_{i} \leq c_{i}^{\text {end }} \text { for all } i \in[1, d],
$$

where $c_{i}$ denotes the $i$-th component of vector $\vec{c}$. In other words, each particle which was mapped to one of these cells is tested for contact with particle $p$.

In the Top-Down method, the small cells, defined in Eq. (2.13), which are almost fully covered by big particles (i.e., no small particles can reside in those cells) can be excluded from the contact search, like for example the cells $(3,4,1)$ and $(3,5,1)$ in Fig. 2.7(top). However, we do not know how to identify such cells efficiently, and therefore, have not implemented this optimization. 


\subsubsection{Performance analysis}

The algorithm is applicable to arbitrary systems, however, to estimate the performance of the algorithm, we restrict ourself to systems that are homogeneous in time and in space. In such a system accurate estimates can be obtained and optimal HGrid parameters can be found theoretically.

To analyse the algorithm two time consuming effects are considered:

1. $T^{c d}$ (collision detection effort) The number of possible contacts that have to be examined more closely. The output of the HGrid-algorithm is a number of possible contacts. Optimum HGrid parameters lead to a low number of possible contacts, because for all these possible contacts a computationally expensive exact geometrical intersection test has to be performed to check if the particles really are in contact.

2. $T^{c a}$ (cells access effort) The number of times information is retrieved from a cell. While the goal of the HGrid is to obtain a list of all possible contacts, it comes at a (computational) cost. This cost is estimated by the number of times information is obtained from a single cell.

To calculated estimates for $T^{c d}$ and $T^{c a}$, consider a system of $N$ polydisperse particles with:

- Random positions $\vec{x}_{p}$ within a $d$-dimensional box at packing fraction $\nu$ (without excluded volume effects).

- Random radii between $r_{\min }$ and $r_{\max }=\omega r_{\min }$, according to a normalized probability density function $f(r)$ (for more details see section 2.2.4).

With these properties the expected mean volume per particle $V_{p}$ can be calculated using:

$$
V_{p}=V_{d} \int_{r_{\min }}^{r_{\max }} r^{d} f(r) d r
$$

where $V_{d}$ is the volume of a $d$-hypersphere of unit radius, i.e., $V_{2}=\pi$ and $V_{3}=(4 / 3) \pi$. So the total volume $V$ of all particles becomes:

$$
V=N V_{p}
$$

Given this volume and the packing fraction $\nu$, the size $A$ of a $d$-dimensional box can be calculated as:

$$
A=\left(\frac{V}{\nu}\right)^{\frac{1}{d}}
$$




\section{Contact detection of arbitrarily polydisperse objects}

Now define $N_{h}$ as the expected number of particles at level $h$ and $N_{h}^{c}$ as the number of cells at this level:

$$
\begin{aligned}
& N_{h}=N \int_{\frac{1}{2} s_{h-1}}^{\frac{1}{2} s_{h}} f(r) d r, \\
& N_{h}^{c}=\left(\frac{A}{s_{h}}\right)^{d}=N \frac{V_{p}}{\nu}\left(\frac{1}{s_{h}}\right)^{d},
\end{aligned}
$$

where $s_{0}=2 r_{\min }$ and $s_{L}=2 r_{\max }$. So the expected average number of particles per cell at level $h, m_{h}$, becomes:

$$
m_{h}=\frac{N_{h}}{N_{h}^{c}}=\frac{\nu s_{h}^{d}}{V_{p}} \int_{\frac{1}{2} s_{h-1}}^{\frac{1}{2} s_{h}} f(r) d r .
$$

It must be noted that the number of particles per cell $m_{h}$ is independent of the total number of particles $N$.

As described in section 2.2.2, the algorithm checks for possible contacts at the level of insertion and at the other levels. For both types of contacts estimates of the number of possible contacts and the number of cells that have to be accessed for are made in the following two sections.

\section{Level-of-insertion search}

At the level of insertion $h, N_{h}$ particles are randomly distributed over $N_{h}^{c}$ cells. Therefore, the number of particles in a specific cell at this level $\left(X_{h}\right)$ is binomially distributed with $N_{h}$ the number of trials and $1 / N_{h}^{c}$ the probability of success. With this assumption the expected number of potential contacts within a single cell is obtained (see Appendix). The number of cells that have to be processed is just equal to the number of cells at this level. Therefore, we obtain:

$$
\begin{aligned}
T_{h}^{c d 1} & =\frac{1}{2} N_{h}^{c} \frac{N_{h}}{N_{h}^{c}} \frac{N_{h}-1}{N_{h}^{c}}=\frac{1}{2} m_{h}\left(N_{h}-1\right) \approx \frac{1}{2} m_{h} N_{h}, \\
T_{h}^{c a 1} & =N_{h},
\end{aligned}
$$

where in the last step of Eq. (2.20) it is assumed that the number of particles at level $h$ is much greater than unity. For possible contacts between neighbouring cells one just has to square the expected numbers of particles in a cell, $m_{h}$, and multiply it by the number of neighbouring cells that have 
to be checked, $n_{c}$, and the total number of cells at the current level, $N_{h}^{c}$ :

$$
\begin{aligned}
& T_{h}^{c d 2}=n_{c} N_{h}^{c} m_{h}^{2}=n_{c} N_{h} m_{h}, \\
& T_{h}^{c a 2}=n_{c} N_{h}^{c} m_{h}=n_{c} N_{h},
\end{aligned}
$$

with $n_{c}=\frac{1}{2}\left(3^{d}-1\right)$, i.e., $n_{c}=4$ in $2 \mathrm{D}$ and $n_{c}=13$ in $3 \mathrm{D}$. We obtain that $T_{h}^{c d 1}, T_{h}^{c a 1}, T_{h}^{c d 2}$ and $T_{h}^{c a 2}$ are all linearly dependent on the number of particles $N_{h}$ at level $h$

\section{Cross-level search}

To estimate the number of potential contacts for the cross-level search, first an estimate of the number of cross-cell checks between particles at hierarchy level $j \neq h$ with particles at level $h$ has to be made. In Appendix, the number of cells at level $j$ that have to be scanned for potential contacts with particles at level $h$ is found to be:

$$
b(j, h)=\frac{\int_{\frac{1}{2} s_{h-1}}^{\frac{1}{2} s_{h}}\left(2 \frac{r}{s_{j}}+2\right)^{d} f(r) d r}{\int_{\frac{1}{2} s_{h-1}}^{\frac{1}{2} s_{h}} f(r) d r},
$$

with (expected) lower and upper limits:

$$
\begin{aligned}
& b_{\text {lower }}(j, h)=\left(2+\frac{s_{h-1}}{s_{j}}\right)^{d}, \\
& b_{\text {upper }}(j, h)=\left(2+\frac{s_{h}}{s_{j}}\right)^{d} .
\end{aligned}
$$

The expected number of cross-level checks and the number of cells that have to be accessed within a cross-level check can easily be calculated.

For the Top-Down algorithm:

$$
\begin{aligned}
& T_{h}^{c d 3}=N_{h} \sum_{j=1}^{h-1} m_{j} b(j, h), \\
& T_{h}^{c a 3}=N_{h} \sum_{j=1}^{h-1} b(j, h),
\end{aligned}
$$


and for the Bottom-Up algorithm:

$$
\begin{aligned}
T_{h}^{c d 3} & =N_{h} \sum_{j=h+1}^{L} m_{j} b(j, h), \\
T_{h}^{c a 3} & =N_{h} \sum_{j=h+1}^{L} b(j, h) .
\end{aligned}
$$

Just as for the level-of-insertion search, we obtain that $T_{h}^{c d 3}$ and $T_{h}^{c a 3}$ are linearly dependent on the number of particles $N_{h}$ at level $h$, for both algorithms.

\section{Total computational work}

The total computational work per level can now be calculated by just summing of its components.

For the Top-Down algorithm:

$$
\begin{aligned}
& T_{h}^{c d}=N_{h}\left(\left(\frac{1}{2}+n_{c}\right) m_{h}+\sum_{j=1}^{h-1} m_{j} b(j, h)\right) \\
& T_{h}^{c a}=N_{h}\left(1+n_{c}+\sum_{j=1}^{h-1} b(j, h)\right)
\end{aligned}
$$

and for the Bottom-Up algorithm:

$$
\begin{aligned}
& T_{h}^{c d}=N_{h}\left(\left(\frac{1}{2}+n_{c}\right) m_{h}+\sum_{j=h+1}^{L} m_{j} b(j, h)\right), \\
& T_{h}^{c a}=N_{h}\left(1+n_{c}+\sum_{j=h+1}^{L} b(j, h)\right) .
\end{aligned}
$$

Note that both $T_{h}^{c d}$ and $T_{h}^{c a}$ are linear in the expected number of particles at level $h, N_{h}$, for both methods, because it was shown in Eq. (2.19) that $m_{h}$ is independent of the number of particles. This means that the complexity of the total algorithm is linearly dependent on the total number of particles $N$, for any number of levels $L$ used. However, depending on the packing fraction and the particle radii distribution function a huge pre-factor in front of $N$, even larger than $N$, can appear when choosing inappropriate HGrid parameters (i.e., cell sizes and number of hierarchy levels).

To find the optimal number of hierarchy levels and their cell-sizes, an estimate of the required computational time that is associated with both types of effects, i.e., collision detection work and cell access work, is required. There- 
fore, the ratio $K$ of time required for a single geometric contact detection over the time required to retrieve information from a cell is introduced. From simulations it is found to be close to $K=0.2$ for spherical particles and not dependent on the particle volume fraction [88]. Therefore, an estimate of the total time required for a contact detection step is found to be:

$$
T=\sum_{h=1}^{L}\left(T_{h}^{c d}+K T_{h}^{c a}\right) .
$$

This result is general in the sense that it describes every possible particle system. However to get a feel for the optimal HGrid parameters, we limit ourself to a single type of particle size probability distribution function.

\subsubsection{Particle size probability distribution functions}

In order to estimate the performance of the HGrid-algorithm, the distribution of particle radii has to be known. To account for all possible radii distributions the previous section used a normalized probability distribution function $f(r)$. The probability to find a particle with radius between $r$ and $r+d r$ is equal to $f(r) d r$. This requires that:

$$
\int_{0}^{\infty} f(r) d r=1 .
$$

No general strategy has been found to determine the optimal HGrid-parameters for a general particle radii probability distribution function. Therefore, throughout the remainder of this paper a (truncated) power law size distribution with a constant exponent $\alpha$ is used:

$$
f(r)=C r^{\alpha} \quad \text { for } \quad r_{\min } \leq r \leq r_{\max },
$$

with

$$
\frac{1}{C}=\int_{r_{\min }}^{r_{\max }} r^{\alpha} d r .
$$

Different values of $\alpha$ have different physical significance, for three-dimensional systems they represent (see also Fig. 2.8):

- $\alpha=0$ : Uniform size (rectangular) distribution, i.e., same number of bigger as smaller particles in intervals $d r$.

- $\alpha=-2$ : Uniform area distribution, i.e., the total surface area of particles with radii between $r_{1}$ and $r_{1}+d r$ is equal to the total surface area of particles with radii between $r_{2}$ and $r_{2}+d r$, etc. 


\section{Contact detection of arbitrarily polydisperse objects}

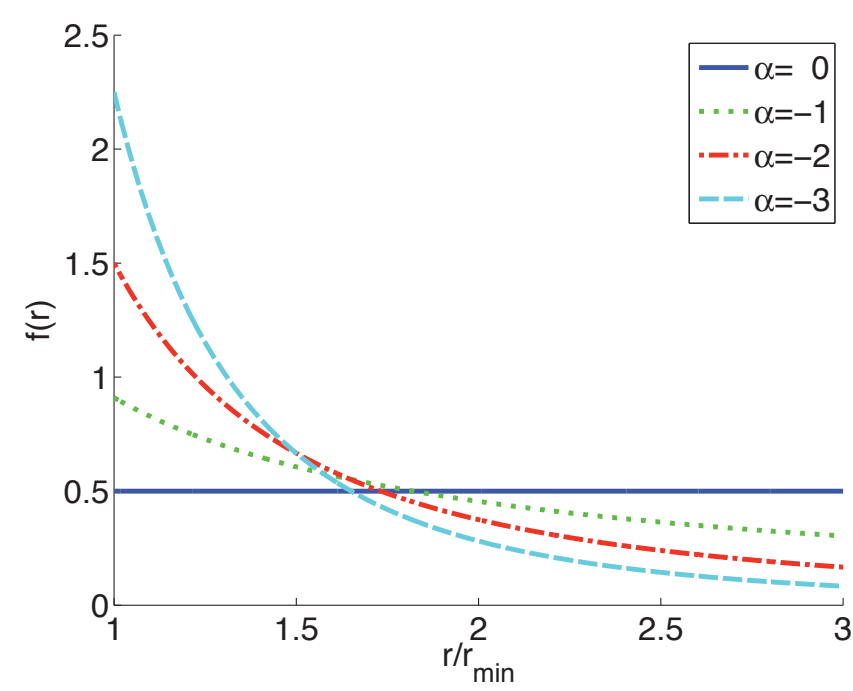

Figure 2.8: Probability density function, Eq. (2.37), for different values of $\alpha$ with $r_{\max } / r_{\min }=3$.

- $\alpha=-3$ : Uniform volume distribution, i.e., the total volume occupied by particles with radii between $r_{1}$ and $r_{1}+d r$ is equal to the total volume occupied by particles with radii between $r_{2}$ and $r_{2}+d r$, etc.

In general, $\alpha>0$ (not used further) implies that there are more bigger particles whereas $\alpha<0$ implies that there are more smaller particles, while $\alpha=0$ corresponds to the same number of small and big particles.

\subsubsection{Cell-sizes distribution}

Having defined the HGrid algorithm, the last thing to do is to decide about the number of hierarchy levels $L$ and the sizes associated with these levels $s_{h}$. In this section four different cell-size distributions are introduced, discussed and compared in order to find optimal HGrid parameters. In the end, the predicted performance of the algorithm is compared against real discrete particle method (DPM) simulations.

\section{Single-level grid}

As a simple reference, we consider the case of a single hierarchy level $(L=1)$, i.e., the Linked-Cell method, and compute the total work $T$ as a function of volume fraction $\nu$, size ratio $\omega$, exponent of the size distribution $\alpha$ and dimension $d$. Due to $L=1$, we have $N_{1}=N$ and $s_{1}=s_{L}=2 r_{\max }=2 \omega r_{\min }$. Using the definition of the number of particles per cell $m_{h}$ from Eq. (2.19) 
and inserting the average particle volume of Eq. (2.14) we obtain

$$
m_{1}=\left(2 \omega r_{\min }\right)^{d} \frac{\nu}{V_{d}} \frac{\int_{r_{\min }}^{\omega r_{\min }} f(r) d r}{\int_{r_{\min }}^{\omega r_{\min }} r^{d} f(r) d r} .
$$

Now substituting the particle radii probability function of Eq. (2.37) and evaluating the integrals results into (for $\alpha \neq-1$ and $\alpha \neq-1-d$ )

$$
m_{1}=(2 \omega)^{d} \frac{\nu}{V_{d}} \frac{1+d+\alpha}{1+\alpha} \frac{\omega^{1+\alpha}-1}{\omega^{1+d+\alpha}-1} .
$$

We are interested in what happens when the polydispersity $\omega$ increases, and thus take the limit of $\omega$ going to infinity:

$$
\lim _{\alpha \rightarrow \infty} m_{1}= \begin{cases}2^{d} \frac{\nu}{V_{d}} \frac{1+d+\alpha}{1+\alpha} & \alpha>-1 \\ -2^{d} \frac{\nu}{V_{d}} \frac{1+d+\alpha}{1+\alpha} \omega^{-1-\alpha} & -1-d<\alpha<-1 . \\ 2^{d} \frac{\nu}{V_{d}} \frac{1+d+\alpha}{1+\alpha} \omega^{d} & \alpha<-1-d\end{cases}
$$

Eq. (2.41) shows that for $\alpha<-1$ the number of particles per cell $m_{1}$ increases with increasing $\omega$. This means that the efficiency of the Linked-Cell algorithm is heavily dependent on $\omega$. This result is also shown in figure 2.9 , where the required computational effort per particle is plotted for different exponents $\alpha$. All curves except for $\alpha=0$ diverge. In general this is true for $\alpha>-1$, meaning that the single-level approach is only appropriate for these values of $\alpha$. In the following sections different distributions of the HGrid cell-sizes using multiple hierarchy levels are tested to find parameters that lead to minimal computation effort.

\section{Multi-level cell-size distribution}

Linear cell-size distribution The easiest method to define the HGrid cell-sizes is to use a linear distribution:

$$
s_{h}=2 r_{\min }\left(1+h \frac{\omega-1}{L}\right) .
$$

Using this cell-size distribution the total work $T$ as a function of the number of HGrid levels $L$ can be calculated. The number of levels where the required computational effort is minimal is chosen as the optimal level and is denoted by $L^{*}$. The minimal work $T$ and the optimal number of levels $L^{*}$ are shown in figure 2.10 for uniform size $(\alpha=0)$ and uniform volume $(\alpha=-3)$ particle size distributions. Comparing the work in figure 2.10(a) with the work for the Linked-Cell method (figure 2.9), it becomes immediately clear that the HGrid algorithm reduces the work significantly. For $\alpha=0$ the improvement is less 


\section{Contact detection of arbitrarily polydisperse objects}

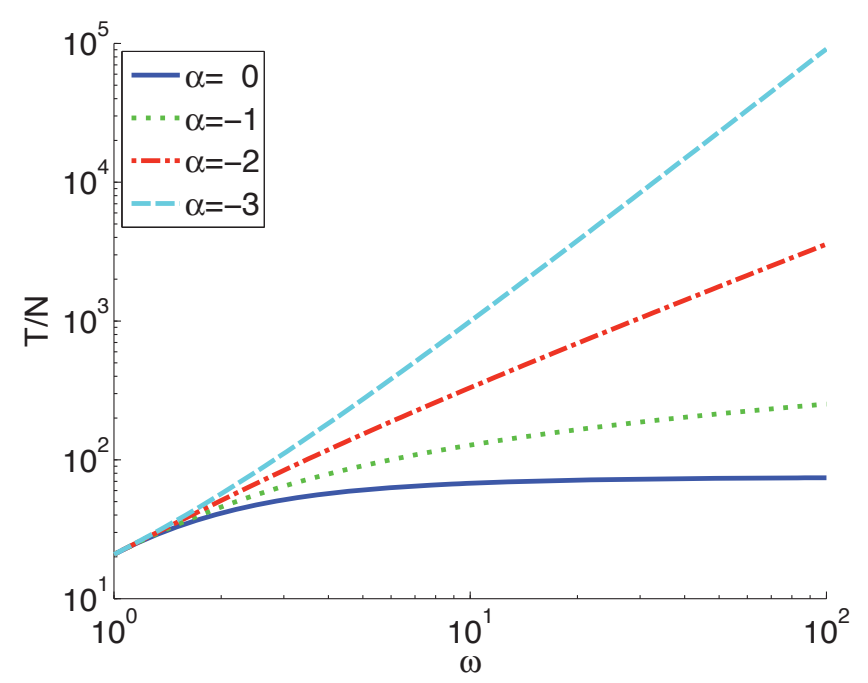

Figure 2.9: Computational effort of the HGrid algorithm using a singlelevel grid (i.e., the Linked-Cell method) as a function of the width of the particle size distribution, $\omega$, for various exponents $\alpha$ using $d=3, \nu=0.7$ and $K=0.2$.

significant, but still the computational effort is reduced by approximately $60 \%$, while for $\alpha=-3$ a speed-up of several orders of magnitude is achieved. Furthermore, while for $\alpha>0$ the Bottom-Up algorithm works slightly better, for $\alpha<0$ the Top-Down algorithm is preferred (data for other values of $\alpha$ not shown). However, in figure 2.10(b) the disadvantage of using a linear cellsize distribution becomes clear. For the $\alpha=-3$ case, the optimal number of levels increases significantly with increasing $\omega$. Therefore, a different cell-size distribution might give better results, as we show in the following sections.

Exponential cell-size distribution To reduce the optimal number of required levels for the HGrid algorithm an exponential cell-size distribution is tested. This distribution stems from the hierarchical tree data structure, where the cell-sizes are usually taken as double the size of the previous lower level of hierarchy. This can be generalized by taking cell-sizes which are defined as

$$
s_{h+1}=q s_{h},
$$

with $q>1$, to make sure that higher level cell-sizes are larger. If one substitutes the boundary conditions $\left(s_{0}=r_{\min }\right.$ and $\left.s_{L}=\omega r_{\min }\right)$, the system of 


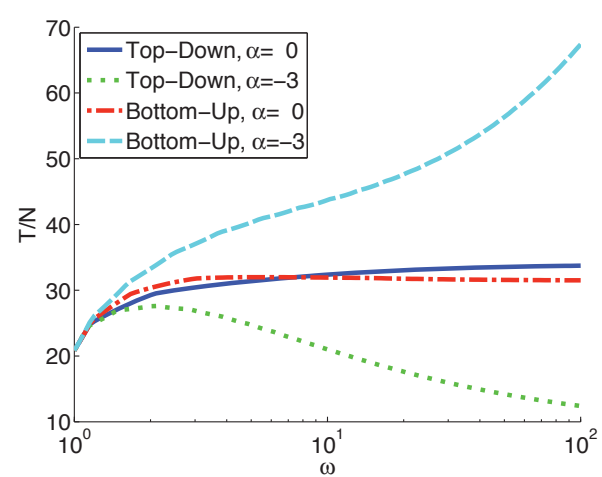

(a) Computational effort.

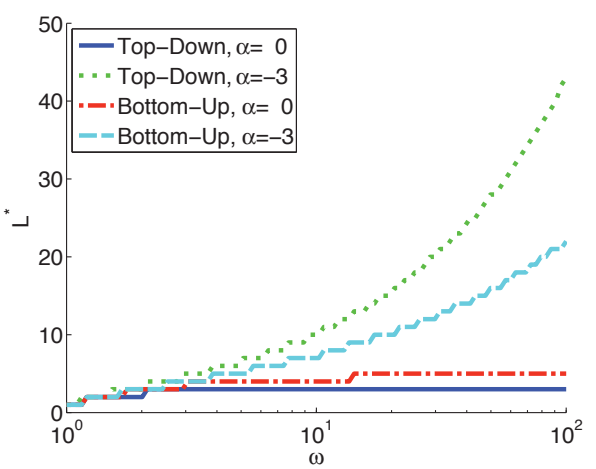

(b) Number of levels.

Figure 2.10: Computational effort and optimal number of levels for the HGrid algorithm with a linear cell-size distribution as a function of the width of the particle size distribution, $\omega$, for various exponents $\alpha$, for both the Top-Down and the Bottom-Up algorithms using $d=3, \nu=0.7$ and $K=0.2$.

equations can be solved analytically:

$$
s_{h}=2 r_{\min } \omega^{\frac{h}{L}} .
$$

As for the linear cell-size distribution, the total work as a function of the number of HGrid levels is calculated and the optimum values are selected and plotted in figure 2.11 for different values of $\alpha$ and $\omega$. For $\alpha=0$ the BottomUp algorithm works better, while for $\alpha=-3$ the Top-Down algorithm is preferred. Data for other values of $\alpha$ (not shown here), indicate that in general for $\alpha \leq-2$ the Top-Down algorithm is preferred. The optimal number of levels is not that strongly dependent on $\omega$ as the linear cell-size distribution, when the Top-Down approach is used. However, there is still a trend in that the optimal number of levels increases with increasing $\omega$ for the Bottom-Up algorithm.

Constant ratio of the number of particles per cell Another approach originates from the idea that it may be beneficial to keep the number of particles per cell fixed at every hierarchy level [89]. This simple idea can easily be extended to a rule where the ratio of particles per cell over two adjacent levels is fixed

$$
m_{h+1}=q m_{h} .
$$

This implies that for $q<1$ the number of particles per cell is decreasing with increasing a hierarchy level, for $q>1$ increasing, and for $q=1$ constant. Using the definition of $m_{h}$ from Eq. (2.19) we can rewrite Eq. (2.45) as 


\section{Contact detection of arbitrarily polydisperse objects}

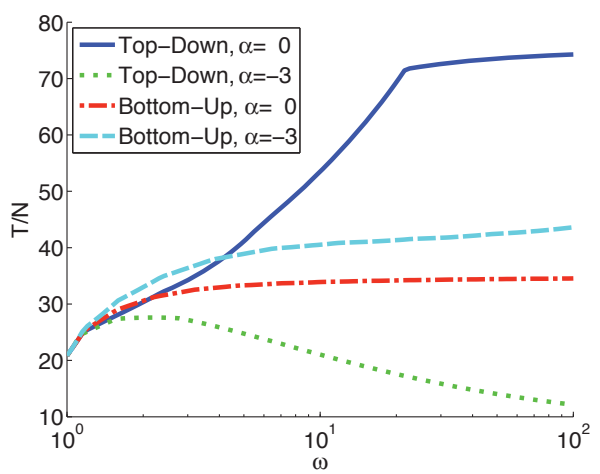

(a) Computational effort

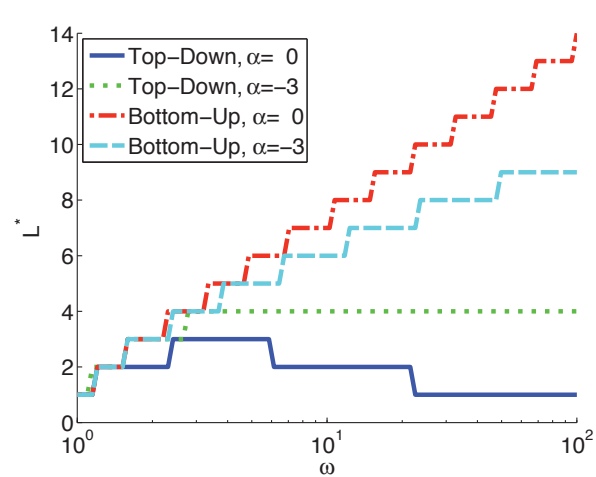

(b) Number of levels.

Figure 2.11: Computational effort and optimal number of levels for the HGrid algorithm with an exponential cell-size distribution as a function of the width of the particle size distribution, $\omega$, for various exponents $\alpha$, for both the TopDown and the Bottom-Up algorithms using $d=3, \nu=0.7$ and $K=0.2$.

follows:

$$
\left.s_{h+1}^{d} F(r)\right|_{s_{h} / 2} ^{s_{h+1} / 2}=\left.q s_{h}^{d} F(r)\right|_{s_{h-1} / 2} ^{s_{h} / 2}
$$

where

$$
F(r)=\int f(r) d r
$$

$s_{0}=r_{\min }$ and $s_{L}=\omega r_{\min }$. This system of equations can be (at least numerically) solved in terms of $s_{h}$, once a size distribution function $f(r)$ is specified.

Using this cell-size distribution we reduce the problem of selecting the number of levels and their sizes to just choosing the number of levels $L$ and the ratio of particles per cell for different hierarchy levels q. In Ref. [89] the hypothesis was given that it is optimal to keep the number of particles per cell at each level constant, or equivalently using $q=1$. To check this hypothesis the computational effort for different values of $q$ as a function of the number of levels $L$ is shown in figure 2.12 for a system with the uniform volume radii distribution $(\alpha=-3)$ using $\omega=100, \nu=0.7, d=3$ and $K=0.2$. We observe that for values for $q$ of 1,2 and 5 the minima in the required computation effort are roughly equal. This is more clearly visible in the inset, where the minimum computational effort is plotted against $q$. The optimal value is somewhere between $q=1$ and $q=2$. The range and number of different levels, for which the computational effort is acceptable, are much bigger for $q=1$ than for $q=2$ and thus it is advised to use $q=1$, for the sake of simplicity. This is also confirmed for different systems parameters and the Bottom-Up algorithm (data not shown).

Using $q=1$, the optimal work and optimal number of levels $L^{*}$ is shown 


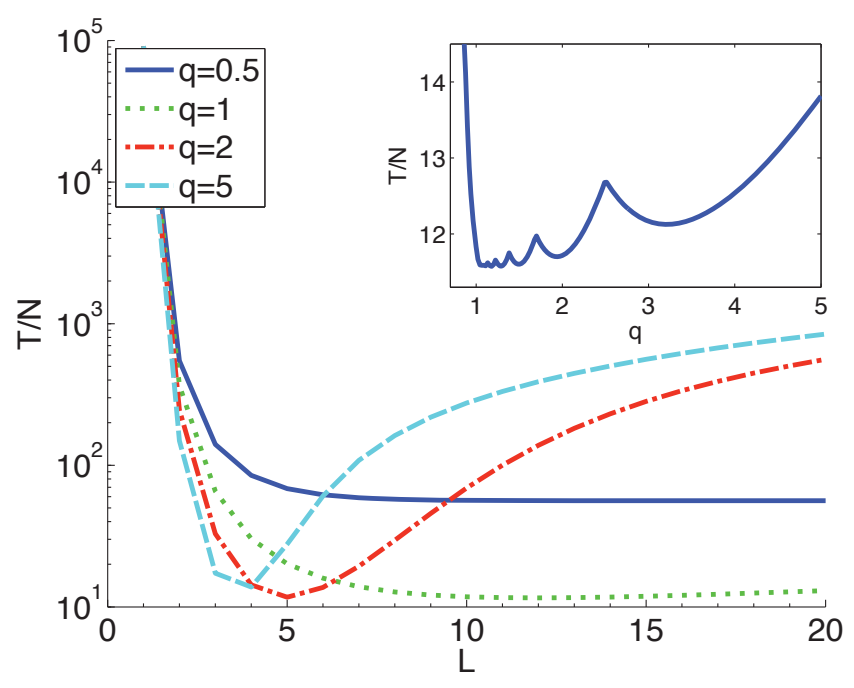

Figure 2.12: Computational effort of the HGrid algorithm with a cell-size distribution, where the ratio of the numbers of particles per cell, $q$, is constant as a function of the width of the particle size distribution, $\omega$, for different number of levels $L$ for the Top-Down method using $\omega=100, \alpha=-3, d=3$, $\nu=0.7, K=0.2$. In the inset the minimum computational effort is shown for different values of $q$.

in figure 2.13. For all values of $\alpha$ the Top-Down algorithm is preferred (data for other values of $\alpha$ not shown).

Optimal cell-size distribution In order to check if the constant number of particles per cell method indeed gives a close-to-optimal result, a numerical optimization method is used to minimize Eq. (2.35) in terms of $L$ and $s_{h}$ under the conditions that $s_{h+1} \geq s_{h}, s_{0}=r_{\min }$ and $s_{L}=\omega r_{\min }$. This is performed using the MATLAB [135] iterative optimization function "fmincon". In this function, a quadratic programming subproblem is solved at each iteration, where the Hessian of the Langrangian at each iteration is calculated using the BFGS algorithm.

The minimal required computational effort $T$ and the optimal number of levels $L^{*}$ for this method are shown in figure 2.14. Note that the results are quite comparable to that of the constant number of particles per cell method in figure 2.13 .

\section{Comparison of the cell-size distribution functions}

In this section the four different cell-size distributions are compared and best practices are given. From figure 2.9 and the analysis of the singlelevel reference case it becomes clear that the HGrid algorithm is essential for 


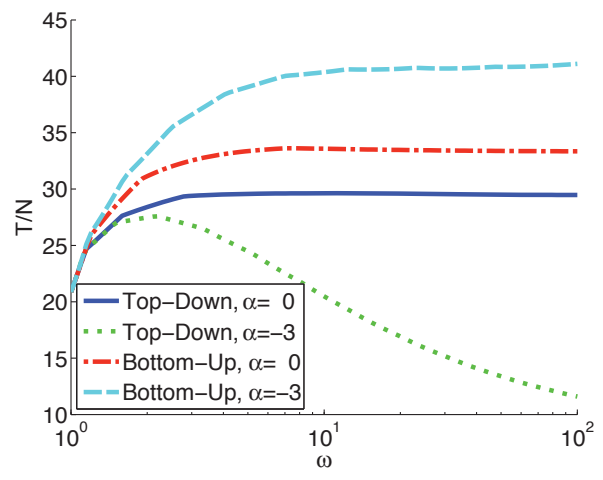

(a) Computational effort.

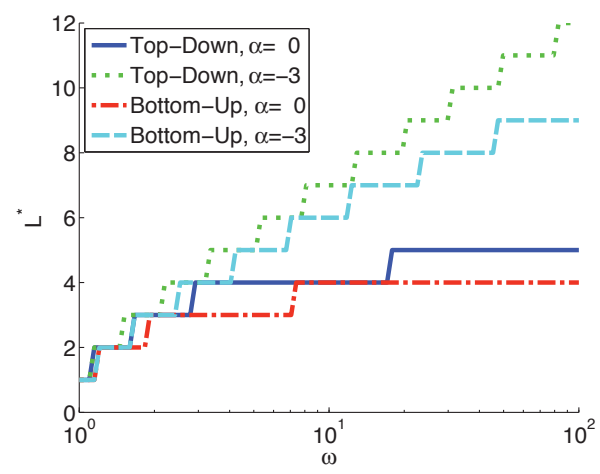

(b) Number of levels.

Figure 2.13: Computational effort and optimal number of levels for the HGrid algorithm with a cell-size distribution, where the number of particles per cell is the same at each level as a function of the width of the particle size distribution, $\omega$, for various exponents $\alpha$, for both the Top-Down and the Bottom-Up algorithms using $d=3, \nu=0.7$ and $K=0.2$.

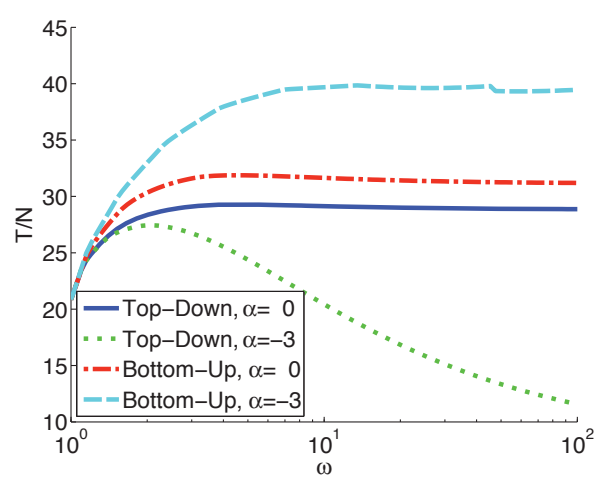

(a) Computational effort.

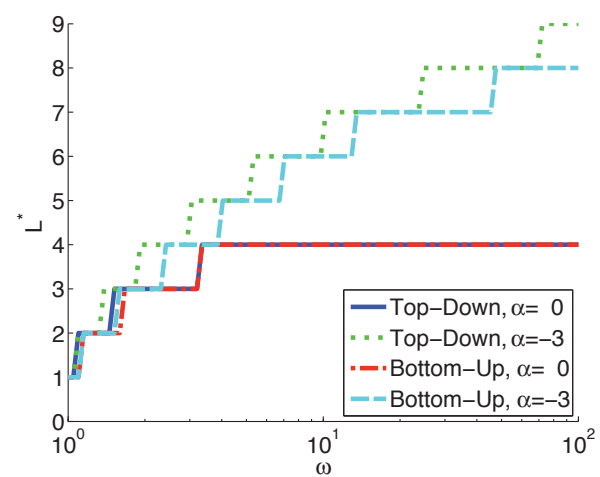

(b) Number of levels.

Figure 2.14: Computational effort and optimal number of levels for the HGrid algorithm with an optimal cell-size distribution as a function of the width of the particle size distribution, $\omega$, for various exponents $\alpha$, for both the TopDown and the Bottom-Up algorithms, using $d=3, \nu=0.7$ and $K=0.2$. 
$\alpha \leq-1$, however, the required optimal parameters are yet to be determined. The required computational effort for different particle distributions using the four cell-size distribution functions is shown in figures 2.9-2.14. All of the used algorithms show a significant decrease in computational effort over the single-level reference case for all parameters of the particle size distribution functions. Even more important, all but one (the Bottom-Up algorithm using a linear cell-size distribution for $\alpha=0$ ) of the test cases show that for large polydispersities (i.e., high values of $\omega$ ) the optimal efficiency of the algorithm is independent on $\omega$. Also the choice of the cell-size distribution functions is not too important as long as the other HGrid parameters are chosen optimally. However, in practice it is often difficult or impossible to calculate optimal parameters in advance, due to changing particles, density or geometries. Therefore, the sensitivity of the algorithm to different parameters becomes important.

The required work for all previously discussed cell-size distributions is shown in figure 2.15 for the case $\omega=100$ and $\alpha=-3$ using the Top-Down algorithm. Again we see clearly that the minima of the four curves are roughly equal $(12.40,11.57,11.60$ and 11,58 respectively), however, the location of the minimum $L^{*}$ and the sensitivity of the work to using suboptimal parameters differ quite a lot. For the linear cell-size distribution the location of the minimum is at $L^{*}=43$ (outside the domain of the figure), which is significantly higher than for the other distributions. Such a high number of levels results in additional overhead, especially for particles with complex geometries, therefore it is not advised to use the linear cell-size distribution. For the exponential cell-size distribution the minimum is located at $L^{*}=4$, however choosing $L=3$ or $L=6$ already decreases the performance by $43 \%$ and $24 \%$ respectively. For the constant number of particles per cell distribution the optimum is located at $L^{*}=12$ and choosing $L=8$ or $L=19$ reduces the performance just by $10 \%$. So, it is advised to either use the constant number of particles per cell or the optimal cell-size distributions, which is even less sensitive to $L \neq L^{*}$.

The values of the cell-sizes $s_{h}$ and the numbers of particles per cell $m_{h}$ for the different cell-size distributions are shown in figure 2.16. We observe that in most cases $m_{h}<1$ is a good choice.

\section{Comparison with simulations}

The estimated computational efficiency of the HGrid algorithm is compared against DPM simulations to check the assumptions used in the derivation. Therefore, different packings of particles are generated, using a combination of event-driven and soft particle methods, for different packing fractions, particle size distributions and number of particles. More specific, we use 


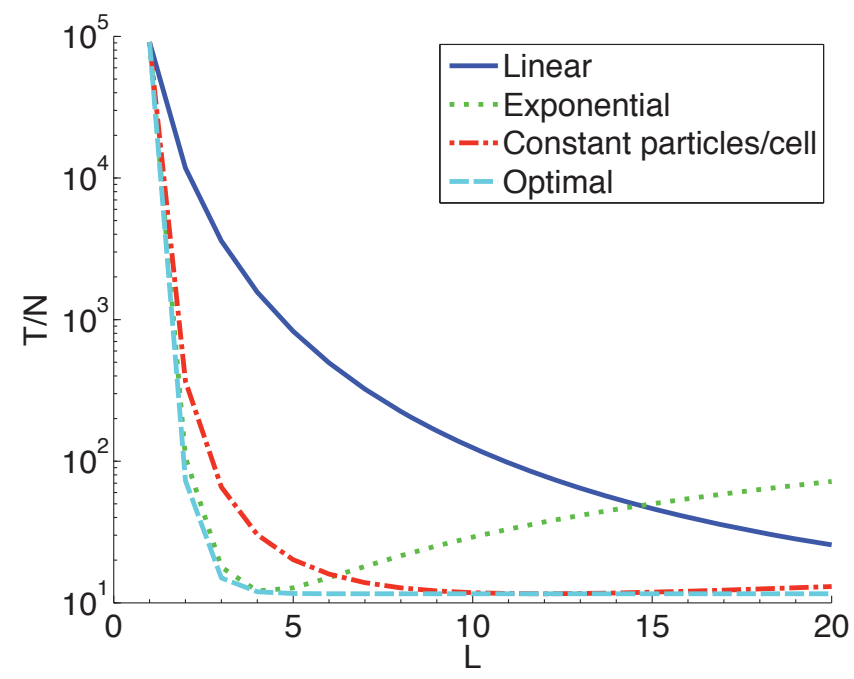

Figure 2.15: Computational effort of the HGrid algorithm as a function of the number of levels, $L$, for different cell-size distributions, using $\alpha=-3$, $\omega=100, d=3, \nu=0.7$ and $K=0.2$.

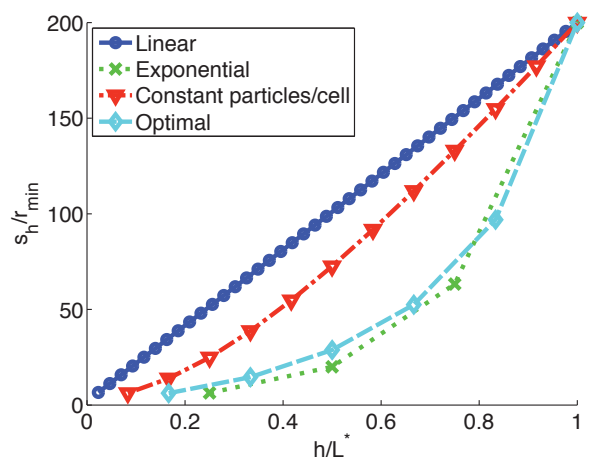

(a) Cell-sizes.

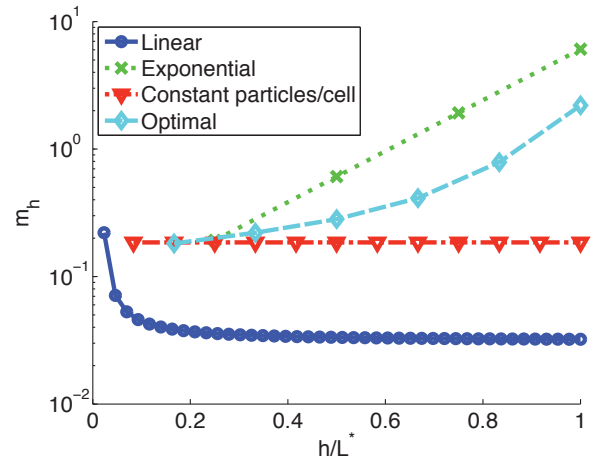

(b) Particles per cell.

Figure 2.16: Cell-sizes and number of particles per cell for the four different cell-size distribution methods with optimal HGrid parameters, using $\alpha=-3$, $\omega=100, d=3, \nu=0.7$ and $K=0.2$. 
homogeneous and isotropic disordered systems of colliding elastic spherical particles in a cubical box with hard walls or periodic boundary conditions (for more detail see Ref. [89]). In MercuryDPM (mercurydpm.org) [92,136,137] a contact detection step has been run using the optimal cell-size distribution, where the number of times a cell is accessed and the number of narrow phase contact detection steps has been counted to compare against the theoretical predictions.

In figure 2.17 the results are shown for one million particles using different packing fractions $\nu$ and different widths $\omega$ of the particle size distribution function. For lower packing fractions the results are extremely accurate, but for higher volume fractions the required work in real simulations becomes slightly higher than expected, however, the overall trend is captured nicely. The main reasons for this deviation we attribute to the excluded volume and finite size effects. In the model derivation the particle centers are assumed to be randomly distributed throughout the domain, whereas in real DPM simulations particles are not allowed to have large overlaps. This is already seen in figure 2.7(top), where large particle B is so big that it completely covers the small grid cells $(3,4,1)$ and $(3,5,1)$. So the numbers of cells where the small particles can be distributed is significantly reduced by the presence of large particles. In the test case for $\alpha=-3$ and $\omega=100$ the percentage of cells on the lowest hierarchy level where two or more particles reside is $2.26 \%$. For randomly distributed particles (with arbitrary overlaps), however, this would only be $1.26 \%$, so a significant increase due to the excluded volume effect. This effect is less pronounced at lower packing fractions because there is more free space.

The same conclusion holds for different number of particles and different shapes of the particle size distribution function, as shown in figure 2.18. The required computational effort is estimated quite nicely. In real DPM simulations for a small number of particles, the real computational work is slightly less than expected from the model. This is due to the fact that a system of infinite size is assumed for the model. When only a finite number of particles is used there will be particles at the boundary of the domain, which will have less neighbouring particles than particles in the middle of the domain. When using more particles, the ratio of particles at the boundary compared to particles in the central part will become lower and thus increasing the computational effort. This dependence has been tested by creating and testing systems with periodic boundary conditions (solid circles in figure 2.18). 


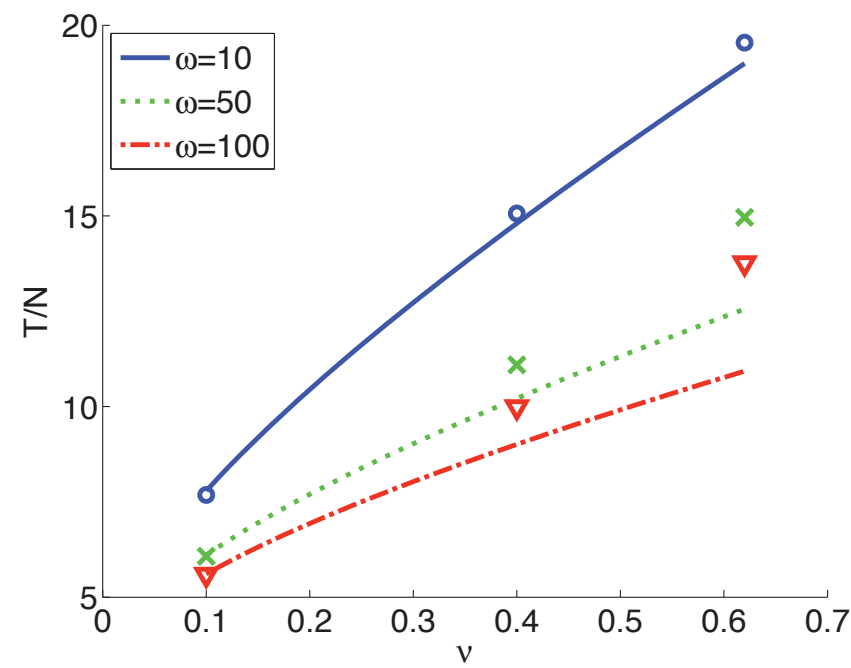

Figure 2.17: Comparison of the estimated HGrid computational effort (lines) versus that for a real DPM system (markers) for different packing fractions and different polydispersities, using $\alpha=-3, N=1000001, d=3, K=0.2$ and Optimal cell-size distribution.

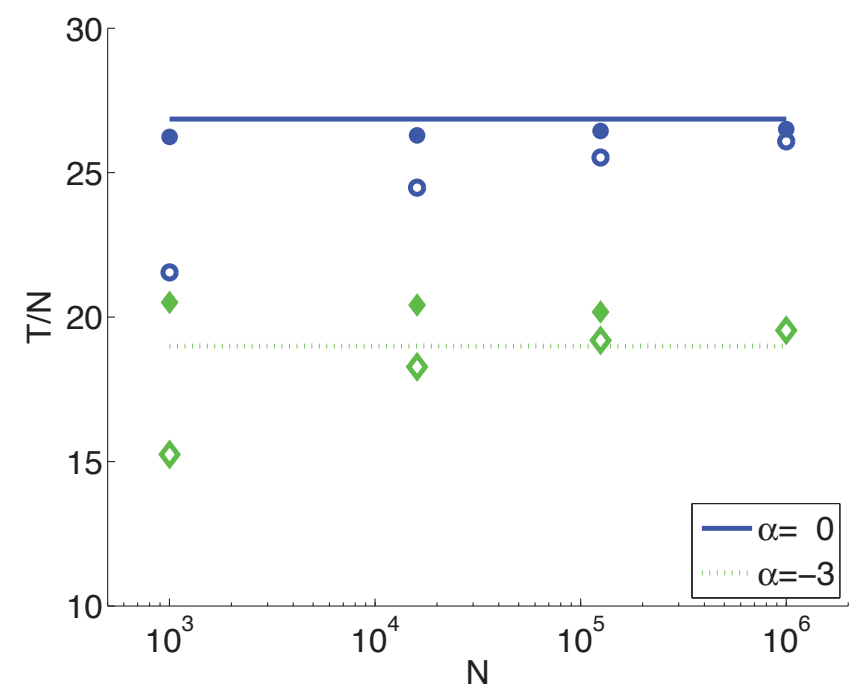

Figure 2.18: Comparison of the estimated HGrid computational effort (lines) versus that for a DPM system (markers) for different packing fractions and different exponents of the particle size distribution function, using $\omega=10$, $\nu=0.62, d=3, K=0.2$ and Optimal cell-size distribution. Open symbols correspond to simulations using solid walls, filled symbols represent systems with periodic boundary conditions. 


\subsubsection{Summary and Conclusion}

Contact detection is a fundamental problem that occurs in many different kind of simulation methods. This process is often computationally expensive, usually taking up a considerable proportion of CPU time, especially for nonuniform (polydisperse) particle size systems.

In this paper, we studied analytically the computational effort of two algorithms for contact detection (i.e., Bottom-Up and Top-Down), based on the multi-level hierarchical grid data structure. The basic idea of these algorithms is the fact, that usually there are lots of particles in the system, which cannot be in touch, as they are too distant. The presented methods save a lot of time by excluding such particles from a detailed and time consuming contact examination and evaluation. The scaling performance of the neighbour searching algorithm based on both the number of particles and the width of the particle size distribution, is of great importance.

As an input for the algorithm, the number of hierarchy levels and their cell-sizes are required, to achieve optimal performance. Therefore, we tested four methods for choosing the hierarchical cell-size distribution (i.e., linear, exponential, constant number of particles per cell and optimal) and compare their theoretical performance for a power law particle size distribution function with exponent $\alpha$. For almost all methods the performance of the algorithm becomes independent of the width of the particle size distribution $\omega$, in contrast to the Linked-Cell method. Even better, the computational effort using the algorithm decreases with increasing $\omega$, or with decreasing $\alpha$, at constant system packing fraction. In general, with optimal parameters, the algorithm is able to find contacts in arbitrarily polydisperse particle systems as fast as the Linked-Cell method finds contacts in purely monodisperse particle systems, i.e., no extra work is required due to polydispersity.

For the linear cell-size distribution the optimal number of hierarchy levels is huge for systems with large polydispersity and $\alpha<0$ (i.e., the systems dominated by small particles). Therefore, for this kind of system, the linear cell-size distribution has high computational overhead and in general does not perform well, especially for particles with complex geometries. The exponential cell-size distribution performs better, however it is very sensitive to the number of hierarchy levels used. So it is not appropriate to use this method in dynamical systems, where the particle size distribution, density or system geometry is changing over time. Both the constant number of particles per cell and the optimal cell-size distribution methods perform well and are not too sensitive to the number of levels, and have low overhead.

For $\alpha \leq-1$ the use of a multilevel grid becomes extremely efficient (i.e., several orders of magnitude faster) as compared to the single level LinkedCell method, if optimal parameters are used. On the other side for $\alpha>-1$ 
the use of a multilevel grid does not present a major advantage (but can improve performance slightly). The contact detection time is estimated to be $T \leq 30 N$ for three-dimensional spherical systems (where a unit of time is defined as the time required for a two-sphere overlap test). Our analysis technique allows to investigate further how the algorithm performs for other size distributions, e.g. log-normal.

\subsubsection{Recommendations}

In this section recommendations are given for setting the HGrid parameters.

- Use the Top-Down algorithm.

- If possible, perform your own minimization using your exact system and appropriate overhead factor $K$ to obtain the optimal number of levels and their cell-size distribution.

- Otherwise, use a cell-size distribution where the number of particles per cell is approximately the same at each level. For the number of levels $L$ we refer to figure 2.13.

\section{Appendix}

\section{Estimated number of contacts within a cell}

To calculate the estimated number of potential contacts within a cell, we assume that at level $h$ there are $N_{h}$ particles randomly divided over $N_{h}^{c}$ cells, such that the probability that a certain particle goes to a certain cell is $1 / N_{h}^{c}$. The number of particles in cell $i, X_{i}$, follows the binomial distribution with parameters $N_{h}$ and $1 / N_{h}^{c}$ such that the probability of finding $n$ particles in cell $i$ is equal to:

$$
P\left(X_{i}=n\right)=\left(\begin{array}{c}
N_{h} \\
n
\end{array}\right)\left(\frac{1}{N_{h}^{c}}\right)^{n}\left(1-\frac{1}{N_{h}^{c}}\right)^{N_{h}-n},
$$

where

$$
\left(\begin{array}{c}
N_{h} \\
n
\end{array}\right)=\frac{N_{h} !}{n !\left(N_{h}-n\right) !}
$$

is the binomial coefficient. If $n$ particles reside in a certain cell, we have to check for $n(n-1) / 2$ potential contacts in that cell and thus we can calculate the estimated number of potential contacts within a single cell by calculating its weighted average. However, we are interested in the potential contacts 
within cells for the whole hierarchy level $h$ and thus have to multiply this by the number of cells at this level $N_{h}^{c}$ to obtain $T^{c d 1}$.

$$
T^{c d 1}=\frac{N_{h}^{c}}{2} \sum_{n=0}^{N_{h}} n(n-1) P\left(X_{i}=n\right) .
$$

First, note that for $n=0$ and $n=1$ the right-hand side equals zero, and thus we can change the summation domain:

$$
T^{c d 1}=\frac{N_{h}^{c}}{2} \sum_{n=2}^{N_{h}} n(n-1)\left(\begin{array}{c}
N_{h} \\
n
\end{array}\right)\left(\frac{1}{N_{h}^{c}}\right)^{n}\left(1-\frac{1}{N_{h}^{c}}\right)^{N_{h}-n} .
$$

Furthermore, by using the definition of the binomial coefficient we obtain:

$$
\left(\begin{array}{c}
N_{h} \\
n
\end{array}\right)=\left(\begin{array}{c}
N_{h}-1 \\
n-1
\end{array}\right) \frac{N_{h}}{n} .
$$

And we can rewrite Eq. (2.50) as:

$$
T^{c d 1}=\frac{N_{h}^{c}}{2} \frac{N_{h}}{N_{h}^{c}} \frac{N_{h}-1}{N_{h}^{c}} \sum_{n=2}^{N_{h}}\left(\begin{array}{c}
N_{h}-2 \\
n-2
\end{array}\right)\left(\frac{1}{N_{h}^{c}}\right)^{n-2}\left(1-\frac{1}{N_{h}^{c}}\right)^{N_{h}-n} .
$$

Substituting $n=a+2$ and $N_{h}=b+2$ gives

$$
\begin{aligned}
T^{c d 1} & =\frac{N_{h}^{c}}{2} \frac{N_{h}}{N_{h}^{c}} \frac{N_{h}-1}{N_{h}^{c}} \sum_{a=0}^{b}\left(\begin{array}{l}
b \\
a
\end{array}\right)\left(\frac{1}{N_{h}^{c}}\right)^{a}\left(1-\frac{1}{N_{h}^{c}}\right)^{b-a} \\
& =\frac{N_{h}^{c}}{2} \frac{N_{h}}{N_{h}^{c}} \frac{N_{h}-1}{N_{h}^{c}}
\end{aligned}
$$

where in the second step the definition of a probability density function is used.

\section{Number of cells for cross-level search}

To estimate the number of potential contacts for the cross-level search, first an estimate of the number of cells that have to be checked for possible crosslevel contacts has to be made. Therefore, consider a particle $p$ at position $\vec{x}_{p}$ with radius $r_{p}$ such that it resides at hierarchy level $h$ and we want to calculate the number of cells at hierarchy level $j$ that have to be checked for possible contacts. This can be calculated from Eq. (2.12), which reads for 
the $x$-direction (note that the directions are statistically independent):

$$
\begin{gathered}
c_{1}^{\text {start }}=\left\lfloor\frac{x_{p}-r_{p}-s_{j} / 2}{s_{j}}\right\rfloor=\left\lfloor\frac{x_{p}-r_{p}}{s_{j}}-\frac{1}{2}\right\rfloor, \\
c_{1}^{\text {end }}=\left\lfloor\frac{x_{p}+r_{p}+s_{j} / 2}{s_{j}}\right\rfloor=\left\lfloor\frac{x_{p}+r_{p}}{s_{j}}+\frac{1}{2}\right\rfloor .
\end{gathered}
$$

So the number of cells that have to be checked in the $x$-direction is, see Eq. (2.13),

$$
a\left(j, x_{p}, r_{p}\right)=\left\lfloor\frac{x_{p}+r_{p}}{s_{j}}+\frac{1}{2}\right\rfloor-\left\lfloor\frac{x_{p}-r_{p}}{s_{j}}-\frac{1}{2}\right\rfloor+1 .
$$

This result holds for a special particle $p$ at position $\vec{x}_{p}$ with radius $r_{p}$ at level $h$. However, we want to calculate the average number of checks. Therefore, we have to integrate $a(j)$ over all possible positions and all possible radii:

$$
b(j, h)=\frac{\int_{\frac{1}{2} s_{h-1}}^{\frac{1}{2} s_{h}}\left(\int_{-\infty}^{\infty} a(j, x, r) g(x) d x\right)^{d} f(r) d r}{\int_{\frac{1}{2} s_{h-1}}^{\frac{1}{2} s_{h}}\left(\int_{-\infty}^{\infty} g(x) d x\right)^{d} f(r) d r} .
$$

where $g(x)$ and $f(r)$ are the probability density distribution functions for position and radius, respectively. First, note that Eq. (2.58) is periodic with respect to the position of the particle $(x)$ with a period $s_{j}$. So, without loss of generality, we can assume that $x$ is uniformly randomly distributed between 0 and $s_{j}$ :

$$
b(j, h)=\frac{\int_{\frac{1}{2} s_{h-1}}^{\frac{1}{2} s_{h}}\left(\int_{0}^{s_{j}} a(j, x, r) \frac{1}{s_{j}} d x\right)^{d} f(r) d r}{\int_{\frac{1}{2} s_{h-1}}^{\frac{1}{2} s_{h}} f(r) d r} .
$$

To evaluate this integral we rewrite Eq. (2.57) by splitting $\frac{r}{s_{j}}+\frac{1}{2}$ into $a+n$, with $n=\left\lfloor\frac{r}{s_{j}}+\frac{1}{2}\right\rfloor$ and $0 \leq a<1$ :

$$
a(j, x, r)=\left(\left\lfloor\frac{x}{s_{j}}+a\right\rfloor-\left\lfloor\frac{x}{s_{j}}-a\right\rfloor+2 n+1\right) .
$$


Note that the following holds

$$
\begin{aligned}
& \left\lfloor\frac{x}{s_{j}}+a\right\rfloor=\left\{\begin{array}{ll}
0 & \frac{x}{s_{j}}<1-a \\
1 & x_{p} \geq 1-a
\end{array},\right. \\
& \left\lfloor\frac{x}{s_{j}}-a\right\rfloor= \begin{cases}0 & \frac{x}{s_{j}} \geq a \\
-1 & x_{p}<a\end{cases}
\end{aligned}
$$

such that we can integrate Eq. (2.59)

$$
b(j, h)=\frac{\int_{\frac{1}{2} s_{h-1}}^{\frac{1}{2} s_{h}}(2 a+2 n+1)^{d} f(r) d r}{\int_{\frac{1}{2} s_{h-1}}^{\frac{1}{2} s_{h}} f(r) d r},
$$

and substitute $a+n=\frac{r}{s_{j}}+\frac{1}{2}$ back:

$$
b(j, h)=\frac{\int_{\frac{1}{2} s_{h-1}}^{\frac{1}{2} s_{h}}\left(2 \frac{r}{s_{j}}+2\right)^{d} f(r) d r}{\int_{\frac{1}{2} s_{h-1}}^{\frac{1}{2} s_{h}} f(r) d r} .
$$

Without knowing the exact particle size distribution function this integral can not be calculated. However, its upper and lower bounds are:

$$
\begin{aligned}
& b_{\text {lower }}(j, h)=\left(2+\frac{s_{h-1}}{s_{j}}\right)^{d}, \\
& b_{\text {upper }}(j, h)=\left(2+\frac{s_{h}}{s_{j}}\right)^{d} .
\end{aligned}
$$





\section{Chapter 3}

\section{Equation of state and jamming density}

\subsection{Poly- versus bi-disperse systems*}

We study bi- and polydisperse mixtures of hard sphere fluids with extreme size ratios up to 100. Simulation results are compared with previously found analytical equations of state by looking at the compressibility factor, $Z$, and agreement is found with much better than $1 \%$ deviation in the fluid regime. A slightly improved empirical correction to $Z$ is proposed.

When the density is further increased, excluded volume becomes important, but there is still a close relationship between many-component mixtures and their binary, two-component equivalents (which are defined on basis of the first three moments of the size-distribution). Furthermore, we determine the size ratios for which the liquid-solid transition exhibits crystalline, amorphous or mixed system structure.

Near the jamming density, $Z$ is independent of the size distribution and follows a-1 power law as function of the difference from the jamming density $(Z \rightarrow \infty)$. In this limit, $Z$ depends only on one free parameter, the jamming density itself, as reported for several different size distributions with a wide range of widths.

\subsubsection{Introduction}

The hard-sphere model can be applied with some success for various physical phenomena and systems, like e.g., disorder-order transitions, the glass transition, dense granular flows and simple gases and liquids [2,116,138-142].

\footnotetext{
*Based on V. Ogarko and S. Luding, "Equation of state and jamming density for equivalent bi- and polydisperse, smooth, hard sphere systems," J. Chem. Phys., vol. 136, no. 12 , p. $124508,2012$.
} 
Kinetic theory describes the behavior of such particle systems, assuming that the particles are infinitely rigid and collisions are instantaneous $[138,143]$, like in the hard-sphere model. In this paper we study the high density limit, where the caging-effect, that is, particles captured by their neighbors, becomes important in the dynamics of the global system and thus a free-volume theory needs to be formulated [144-150]. There exists no unique equation of state, valid for the intermediate densities, where the system changes from the disordered to the ordered state, since the system displays hysteresis and rate-dependence; for various theoretical approaches see Refs. [116,151-160] and references therein.

In this study systems of particles of many different sizes are investigated in the high-density limit using theory and simulations. Fluid and jammed configurations of hard sphere mixtures are examined for various size distributions at slow compression rates $[66,67,161,162]$. Several authors proposed theories to compute the amorphus jamming density of binary and polydisperse hard sphere mixtures, see Refs. [163-167] and references therein. We construct a simple but physically reasonable model that relates the behavior of different hard sphere mixtures, even for metastable states. Additionally, we give accurate data for the jamming density as a function of size polydispersity, which is important for e.g. experiments on non-monosized colloidal or granular systems.

In section 3.1.2, the theoretical ideas are introduced, which are needed to analyze the numerical results presented in section 3.1.3, before the results are summarized in section 3.1.4.

\subsubsection{Theory}

We consider a $s$-component thermalized mixture of $N$ elastic smooth spheres, homogeneously placed in three-dimensional (3D) systems of volume $V$. Spheres are located at positions $\mathbf{r}_{i}$ with velocities $\mathbf{v}_{i}$, radii $a_{i}$ and masses $m_{i}$. The kinetic energy $E_{k}=(1 / 2) \sum_{i=1}^{N} m_{i} \mathbf{v}_{i}^{2}$ is dependent on time via the particle velocities $\mathbf{v}_{i}$. For rigid spheres that do not interact except via an infinite repulsion on contact, i.e., with zero interaction / contact duration, the total energy is given by $E=E_{k}$, whereas for soft spheres the potential energy also has to be considered. The temperature in equilibrium hard-sphere systems is not a relevant parameter, since it does not affect the equilibrium configuration [67], it only scales time (or the free energy [28]). As a consequence, there is only one independent thermodynamic state variable, which can be either the reduced (dimensionless) pressure (so-called compressibility factor) $Z=P V / N k_{B} T$ or the density (volume fraction) $\nu=4 \pi \sum a_{i}^{3} /(3 V)$, related through the equation of state (EOS), where $k_{B} T=2 E /(3 N)$ with the Boltzmann constant $k_{B}$. 


\section{Bidisperse systems}

Let us consider a binary $(s=2)$ mixture of spheres with radii $a_{1}$ and $a_{2}$, with $N_{1}$ and $N_{2}$ the number of particles of each kind, and $N=N_{1}+N_{2}$. Thus the mixture can be classified by only two parameters, the composition $n_{1}=N_{1} / N$ and the size ratio $R=a_{1} / a_{2}$. The total volume fraction $\nu=$ $\nu_{1}+\nu_{2}$ is the last relevant system parameter, since the partial volume fractions $\nu_{1,2}=4 N_{1,2} \pi a_{1,2}^{3} /(3 \mathrm{~V})$ can be expressed [158] in terms of $n_{1}$ and $R$, using the dimensionless moments

$$
A_{k}=n_{1}+\left(1-n_{1}\right) R^{-k}=\left\langle a^{k}\right\rangle / a_{1}^{k},
$$

where $\left\langle a^{k}\right\rangle=n_{1} a_{1}^{k}+n_{2} a_{2}^{k}$. With this, one has $\nu_{1}=n_{1} \nu / A_{3}$ and $\nu_{2}=$ $\left(1-n_{1}\right) \nu /\left(R^{3} A_{3}\right)$.

A calculation in style of Jenkins and Mancini, see Refs. [118,168], leads to the partial translational pressures $p_{i}^{t}=2 n_{i} E /(3 V)$ for species $i$ and to the collisional pressures $p_{i j}^{c}=\pi N_{1} N_{2} g_{i j} a_{i j}\left(1+r_{i j}\right) T /\left(3 V^{2}\right)$ with the particle correlation function $g_{i j}$ evaluated at contact, and $a_{i j}=a_{i}+a_{j}$. In the following, for simplification the inter-species restitution coefficients $r_{i j}$ are set to unity (elastic case), $r_{11}=r_{12}=r_{22}=1$. The particle correlation functions $g_{i j}$ from Ref. $[57,118,169,170]$, are here expressed in terms of $A_{2,3}$, $R$ and $\nu$ :

$$
\begin{gathered}
g_{11}=\frac{1}{1-\nu}+\frac{3 \nu \frac{A_{2}}{A_{3}}}{2(1-\nu)^{2}}+\frac{\left(\nu \frac{A_{2}}{A_{3}}\right)^{2}}{2(1-\nu)^{3}}, \\
g_{22}=\frac{1}{1-\nu}+\frac{3 \nu \frac{A_{2}}{R A_{3}}}{2(1-\nu)^{2}}+\frac{\left(\nu \frac{A_{2}}{R A_{3}}\right)^{2}}{2(1-\nu)^{3}}, \\
g_{12}=\frac{1}{1-\nu}+\frac{3 \nu \frac{A_{2}}{(1+R) A_{3}}}{(1-\nu)^{2}}+\frac{2\left(\nu \frac{A_{2}}{(1+R) A_{3}}\right)^{2}}{(1-\nu)^{3}} .
\end{gathered}
$$

Thus, the global pressure in the mixture is:

$$
\begin{aligned}
p^{m}= & p_{1}^{t}+p_{2}^{t}+p_{11}^{c}+2 p_{12}^{c}+p_{22}^{c} \\
& =\frac{2 E}{3 V}\left[1+\frac{\nu}{2\left\langle a^{3}\right\rangle}\left(g_{11} a_{11}^{3} n_{1}^{2}+2 g_{12} a_{12}^{3} n_{1} n_{2}+g_{22} a_{22}^{3} n_{2}^{2}\right)\right] \\
& =\frac{2 E}{3 V}\left[1+4 \nu g_{\mathrm{eff}}(\nu)\right] .
\end{aligned}
$$

Like done for 2D systems in Ref. [158], the effective correlation function $g_{\text {eff }}(\nu)$ can be expressed in term of the dimensionless moments:

$$
O_{1}=\frac{\langle a\rangle\left\langle a^{2}\right\rangle}{\left\langle a^{3}\right\rangle} \text { and } O_{2}=\frac{\left\langle a^{2}\right\rangle^{3}}{\left\langle a^{3}\right\rangle^{2}}
$$


so that (see Appendix)

$$
g_{\mathrm{eff}}(\nu)=\frac{(1-\nu)^{2}+3 O_{1}(1-\nu)+O_{2}(3-\nu) \nu}{4(1-\nu)^{3}} .
$$

Then the equation of state for mono- and bidisperse systems reads:

$$
Z=1+4 \nu g_{\mathrm{eff}}(\nu) \text {. }
$$

Note that in the monodisperse case all $g_{i j}$ and $g_{\text {eff }}(\nu)$ are identical to the pair distribution function at contact $g_{\mathrm{CS}}(\nu)=(1-\nu / 2) /(1-\nu)^{3}$, proposed by Carnahan and Starling (CS) [171], since $R=1, A_{k}=O_{1,2}=1$ (using the relation $Z_{\mathrm{CS}}=1+4 \nu g_{\mathrm{CS}}($ Ref. [57])). The Carnahan-Starling pair distribution function is quite accurate at low and moderate volume fractions, but does not show the reported divergence due to excluded volume effects at the close packing volume fraction $[172,173]$.

\section{Polydisperse systems}

In the case of a polydisperse mixture $(s=N \rightarrow \infty)$ in which the sphere radius is distributed according to some probability density function $f(a)$, $\left\langle a^{k}\right\rangle \equiv \int a^{k} f(a) d a$ is the $k$-th moment of the size distribution. By just using these moments, the function $g_{\text {eff }}(\nu)$ from Eq. (3.7) is well defined also for multi-component systems of spheres. It turns out that the equation of state (3.8) coincides with the well-known Boublík, Mansoori, Carnahan, Starling, and Leland (BMCSL) equation of state proposed for mixtures [57, 58]:

$$
Z_{\mathrm{BMCSL}}=\frac{1}{1-\nu}+O_{1} \frac{3 \nu}{(1-\nu)^{2}}+O_{2} \frac{\nu^{2}(3-\nu)}{(1-\nu)^{3}} .
$$

There are several modified equations of state for multi-component mixtures in the literature, which require the knowledge of the equation of state for a one-component system [155]. First, we consider Santos et al.'s equation of state, based on the Carnahan-Starling equation of state:

$$
Z_{\mathrm{SCS}}=Z_{\mathrm{BMCSL}}+\left(O_{1}-O_{2}\right) \frac{\nu^{3}}{(1-\nu)^{3}} .
$$

Second, following Santos et al.'s procedure based on the Carnahan-StarlingKolafa (CSK) equation of state, $Z_{\mathrm{CSK}}=\left[1+\nu+\nu^{2}-2 \nu^{3}(1+\nu) / 3\right] /(1-\nu)^{3}$ [174], one gets

$$
Z_{\mathrm{SCSK}}=Z_{\mathrm{SCS}}+\left(O_{1}+O_{2}\right) \frac{\nu^{3}(1-2 \nu)}{6(1-\nu)^{3}}
$$


Third, Boublík extended the CSK equation of state to mixtures [175], yielding:

$$
Z_{\mathrm{BCSK}}=Z_{\mathrm{BMCSL}}+\mathrm{O}_{2} \frac{\nu^{3}(1-2 \nu)}{3(1-\nu)^{3}} .
$$

Although we could consider the extensions of other equations of state [176], for the sake of simplicity we will restrict our analysis to the above mentioned equations of state.

\section{Discussion}

It is interesting to observe that all the equations of state considered in section 3.1.2 are functionals of the particle size distribution (PSD) only through its dimensionless moments $O_{1}$ and $O_{2}$. Therefore, we study and discuss the properties of $O_{1}$ and $O_{2}$. It can be shown that $0<O_{1} \leq 1$ and $O_{1}^{2} \leq O_{2} \leq O_{1}$ for any size distribution function (see Appendix). This property makes it convenient to characterize a size distribution by a point on the $O_{1} O_{2}$ plane.

We can see that $O_{1}$ is the ratio of the arithmetic mean diameter to the Sauter mean diameter, and it is a measure of the broadness of the PSD [177], i.e., $O_{1}=D_{10} / D_{32}$, with $D_{p q}=2\left(\int a^{p} f(a) d a / \int a^{q} f(a) d a\right)^{1 /(p-q)}$. Correspondingly, $O_{2}=\left(D_{20} / D_{32}\right)^{2}$ is another measure for shape and width of the PSD. Those combined size-descriptors were already used as early as 1979 to model systems like polydisperse sprays [178].

In the bidisperse case the system of equations (3.6) can be solved analytically in terms of the variables $n_{1}$ and $R$, yielding a unique solution: $n_{1}^{\text {bi }}\left(O_{1}, O_{2}\right)$ and $R^{\mathrm{bi}}\left(O_{1}, O_{2}\right)$ (see Appendix). This means that for any given polydisperse system we can construct an "equivalent" bidisperse system, which has the same equation of state in the fluid regime. We use this in section 3.1.3 to check how the compressibility factor and the jamming density of polydisperse systems are related to those of their bidisperse equivalents. Earlier, equivalent binary mixtures were used to investigate a slightly polydisperse hard sphere crystal in Ref. [52], while here we will show that they can be used to model widely polydisperse fluids and glasses at all densities, which is in the spirit of what Santos et al. call the "universality" ansatz, see Refs. [60,61,179] and references therein.

\subsubsection{Comparison with simulations}

Since we are interested in the behavior of rigid particles, we perform eventdriven molecular dynamics simulations using the modification of the LubachevskyStillinger algorithm [81] as the primary tool for our investigations $[67,134$, 180]. The system consists of a cubic cell, with periodic boundary conditions, which are imposed to simulate an infinite system, i.e., a statistically 
homogeneous medium. The compressibility factor is calculated from the total exchanged momentum in all interparticle collisions during a certain short time period $\Delta t$, given by 400 events per particle. By growing the size of the particles linearly in time with a growing rate $\Gamma$ one can change the volume fraction (for details see Appendix). Over time the additional energy created during collisions would accelerate the particles, but this is avoided by periodic rescaling of the average particle velocity to hold the mean temperature constant [180], such that the total change in kinetic energy of the system is kept small (below 1\%). If the growing is sufficiently slow, the system will be in approximate equilibrium during the densification and one can rather efficiently gather quasi-equilibrium data as a function of density $[116,181]$. The number of particles $N$ used in most simulations is $16^{3}=4096$, if not explicitly stated otherwise. In order to improve the performance of neighborhood search the ideas of a multilevel contact detection algorithm [89] can be used, but were not implemented here.

\section{Particle size distributions}

The following types of particle size distributions are used: (i) uniform size distribution; (ii) uniform volume distribution, i.e., the probability distribution of the volume of the particles is constant; (iii) systems constructed by mapping the aforementioned polydisperse systems to bidisperse ones (see section 3.1.2 for details).

For a given size distribution, we denote the ratio between the maximum and the minimum particle radius as $\omega$. Therefore, $\omega=1$ corresponds to the monodisperse case, i.e., all sizes are equal, and in our convention $\omega \geq 1$. Further in the paper when we use $\omega$ for bidisperse systems we mean the extreme size ratio of the equivalent polydisperse system, while for the true size ratio we use $R^{\text {bi }}$. Examples and analytical expressions for $O_{1}$ and $O_{2}$ as function of $\omega$ for the considered size distributions are shown in Appendix.

The use of a single variable, i.e., the size ratio $\omega$, excludes continuous distributions like log-normal, for which one has to use $O_{1}$ and $O_{2}$ for classification, see Appendix. The size-distributions used here, due to their sharp edges with well defined $\omega$, could be obtained by ideal sieving from wider, smooth continuously distributed realistic distributions.

The sizes of particles of polydisperse systems are drawn from the particle size distribution function using the systematic sampling approach $[112,113]$. It guarantees a more evenly spread sample, i.e., there will always be large particles, even if they are rare, like in the case of the uniform volume distribution. 


\section{Equation of state in the fluid regime}

In Fig. 3.1 we show the compressibility factor $Z$ from simulations scaled by the BMCSL, SCS, SCSK and BCSK equations of state for different densities and for different size distributions. Note, that in the monodisperse case $\left(O_{1}=O_{2}=1\right) Z_{\mathrm{CS}}=Z_{\mathrm{BMCSL}}=Z_{\mathrm{SCS}}$ and $Z_{\mathrm{SCSK}}=Z_{\mathrm{BCSK}}$.
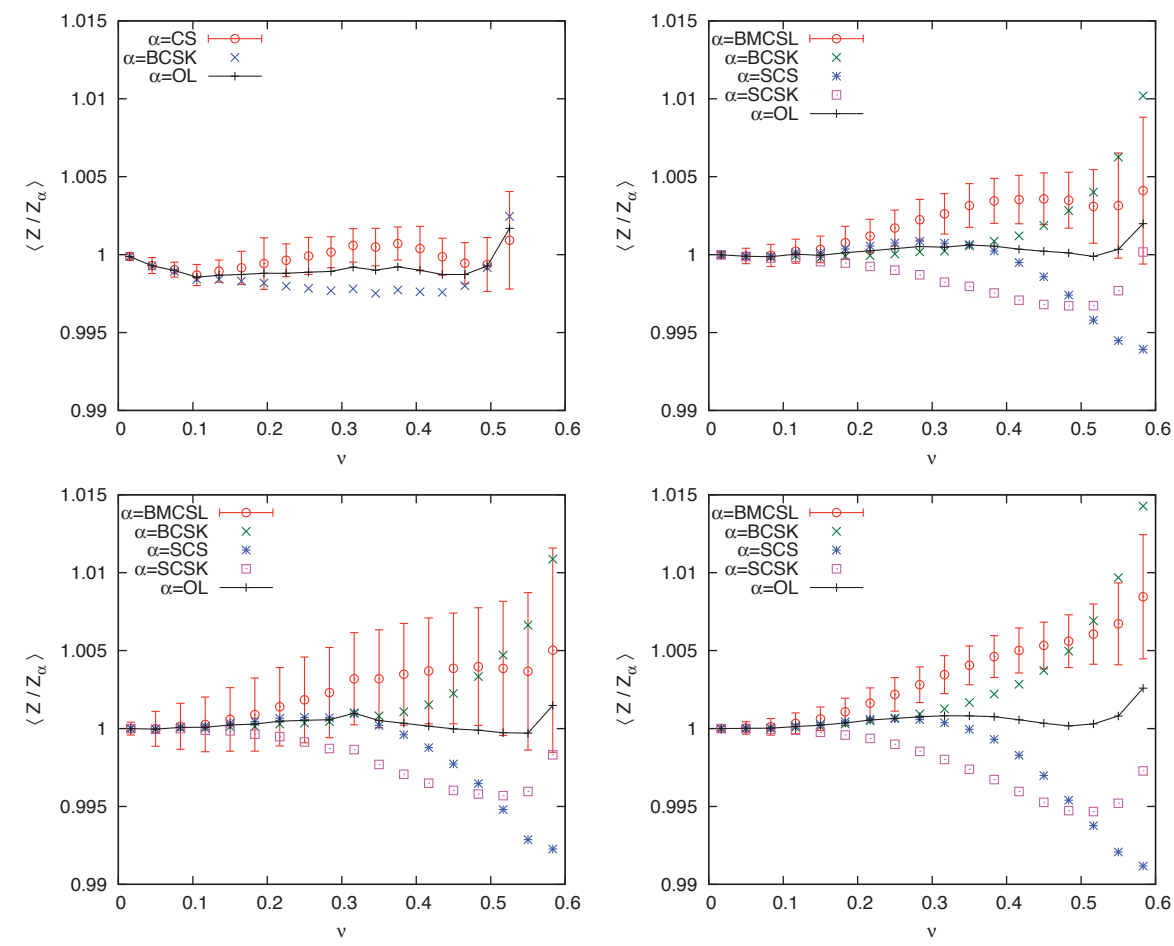

Figure 3.1: The quality factor, i.e., the numerical $Z$ scaled by the theoretical predictions for different densities and for different size distributions (a, top left) monodisperse, and polydisperse with (b, top right) uniform size, $\omega=5$, (c, bottom left) uniform size, $\omega=100$, and (d, bottom right) uniform volume, $\omega=4$. The growth rate for all data is $\Gamma=16 \times 10^{-6}$. The error bars indicate the standard deviation of the quality factor within an averaging bin. The error bars are shown only for the BMCSL EOS since in the other cases they have the same trend and magnitude.

We observe for monodisperse systems that the deviation of the compressibility factor from all considered theories is below $0.3 \%$ (a) in the volume fraction region of $0<\nu<0.54$. The deviation is well below $1 \%$ in the volume fraction region of $0<\nu \leq 0.6$ for polydisperse systems: for uniform size, with $\omega=5$ (b), uniform size, with $\omega=100$ (c), and uniform volume, with $\omega=4$ (d), distributions, respectively. This indicates that the number 
of particles is large enough to realistically represent the respective size distributions to suppress possible effects of finiteness of the system. The uniform volume distribution does not allow to properly realize wider distributions, due to the small sample size, $N=4096$.

Based on all these results, we propose a more accurate equation of state for the fluid regime, which is simply the arithmetic average of Santos et al.'s and Boublík's extensions:

$$
Z_{\mathrm{OL}}=\frac{1}{2}\left(Z_{\mathrm{SCS}}+Z_{\mathrm{BCSK}}\right)
$$

Equation (3.13) is more accurate at intermediate volume fractions $0.20-0.60$ and is almost identical to the other forms for lower volume fractions. For all systems, with $\nu \leq 0.60$, presented in Fig. 3.1, $Z_{\mathrm{OL}}$ performs better than $0.2 \%$ except for the monodisperse case data, for $\nu>0.54$, due to crystallization.

Our simulations also confirm that for $\nu<0.54$ the agreement in the compressibility factor between the considered polydisperse systems and their bidisperse equivalents (defined in section 3.1.2) is of order of $0.1 \%$ for $\omega \leq 50$ (data not shown).

In the following we show what happens at higher volume fractions.

\section{How much disorder is necessary to avoid order?}

According to Alder et al. [10,67], monodisperse hard-sphere systems undergo a first-order fluid-solid phase transition, characterized by a melting point, i.e., the density at which the crystal thermodynamically begins to melt, and a freezing point, i.e., the density at which the fluid thermodynamically begins to freeze. In this section, we investigate how much polydispersity is needed to avoid partial crystallization, complementing an earlier study in 2D (Ref. [159]) and 3D (Ref. [182]) as also studied in experiments on colloids [183].

Since the compressibility factor increases very rapidly at densities higher than the freezing point, we plot the estimated jamming density $\phi_{J}=\nu /(1-$ $d / Z$ ) instead of the compressibility factor, inspired by [67], as shown in Fig. 3.2 , since $\phi_{J}(Z \rightarrow \infty)=\nu$. The estimated jamming density $\phi_{J}$ here is derived from the free-volume EOS for a $d$-dimensional system [147]:

$$
Z_{\mathrm{fv}}=\frac{d}{1-\nu / \phi_{J}}
$$

In Fig. $3.2, \phi_{J}$ is plotted against $\nu$ for different systems with uniform size distribution. Data agree perfectly with the fluid theory before the particle system changes from the fluid state to the state of coexistence of fluid and solid, as indicated by a sharp increase (jump) above the fluid prediction, see Fig. 3.2(a). The jumps are due to partial crystallization observed for size 
ratios $1 \leq \omega \lesssim 1.2$ but not for larger $\omega$. (It must be noted that for the system with $\omega=1.2$, partial crystallization is observed not for every run, as shown in Fig. 3.2(a) for two runs with random initial velocities, i.e., from six runs we found that four systems exhibit partial crystallization, while two systems do not.) Near the critical size ratio, $\omega_{c} \approx 1.20 \pm 0.02$, where ordering effects disappear, also the history and fluctuations of the system play an important role.

For the critical polydispersity, a simple model which agrees with our findings was proposed [24], inspired by Lindemann's melting criterion: a crystal melts when the thermally-induced root-mean-square displacement of an atom or particle reaches a characteristic fraction of the typical interparticle spacing [2]. Our findings also agree with the criterion for stability of the polydisperse crystal based on free energy minimization [35].

It should be noted that it is possible to observe a polydisperse crystal also for large size ratios, if the particles are allowed to redistribute for very long time $[28,184]$. In this case, different annealed phase behavior is expected at high densities [35]. Self-diffusion can occur and spheres can crystallize into multiple crystal phases (domains) each containing spheres of a different size.

In the monodisperse case the metastable freezing point, i.e., the density of partial crystallization of the densified fluid, is very close to 0.54 , which is in good agreement with data from Refs. $[67,116,186]$. This should not be confused with the freezing point, which implies the stable thermodynamic point and occurs at about 0.49 as determined from a number of different simulations $[11,187]$ and free energy calculations [86]. While a metastable freezing point depends on the compression rate, the freezing point does not. The location of the metastable freezing point, $\nu_{f}$, is shifted to higher density with increasing polydispersity. Taken from a few representative simulations, values are $\nu_{f}=0.540,0.548,0.556,0.557,0.565,0.573$ for $\omega=1,1.1,1.12,1.14,1.16,1.2$, respectively, fluctuating with different runs $( \pm 0.005)$. The latter trend reverses (decreasing $\left.\nu_{f}\right)$ for another type of crystallization which we observe in the bidisperse systems with (relatively) large size ratios, see Fig. 3.3. In contrast, there is no strong dependence of the (final) jamming density on $\omega$ for partially crystallized systems, since crystallization is a stochastic nucleation process, i.e., different runs of the same system with random initial particle velocities will lead to different jamming densities (data not shown). The trends in Fig. 3.2(b) show that the larger $\omega$, the larger the density at which disordered systems (not crystallized) deviate from the fluid theory (the sharp increase of $\phi_{J}$ relative to the fluid theory turns to a smooth decrease). In addition, for such systems the jamming density is increasing with $\omega$. Finally, all curves end at the identity line $\phi_{J}=\nu$ at 

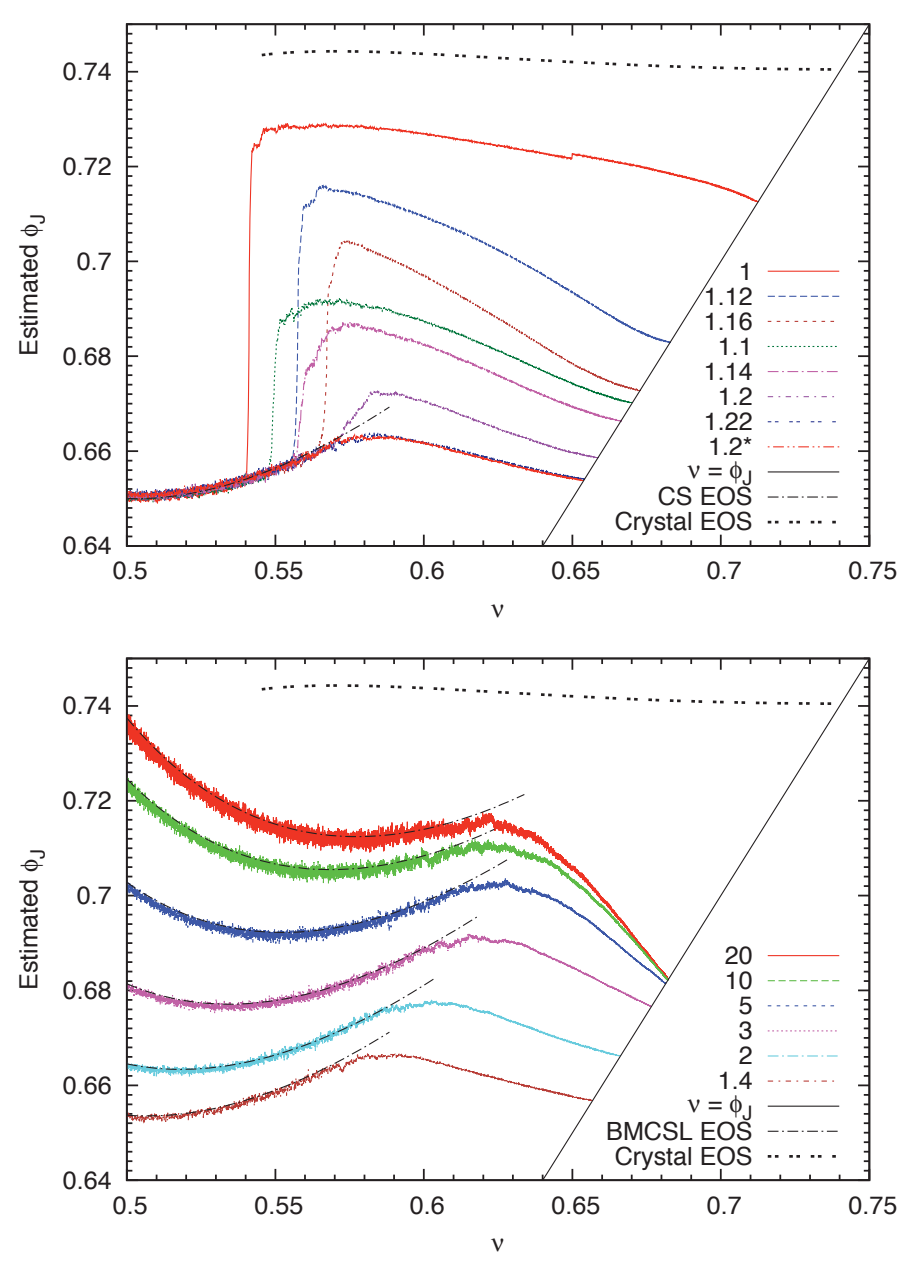

Figure 3.2: The estimated jamming density $\phi_{J}$ as a function of volume fraction $\nu$ for systems with uniform size distribution. Shown are systems of 4096 spheres with various size ratios $\omega$. In (a, top) $\omega$, given in the inset, corresponds to decreasing $\phi_{J}$ (top-to-bottom), and in (b, bottom) increasing $\omega$ corresponds to bottom-to-top. Also plotted are the fluid theory (BMCSL EOS) and the approximation for the crystal phase [185]. The used growth rate here is $\Gamma=8 \times 10^{-6}$. For $\omega=1.2$ data for two different runs are shown, marked with 1.2 and $1.2^{*}$.

densities well below the perfect crystal EOS [185], for which $\phi_{J}=\nu \approx 0.74$ would be expected (for mono-disperse systems).

We checked (data not shown) that the jumps in $\phi_{J}$ vanish around $\omega \approx 1.2$ $\left(R^{\mathrm{bi}} \approx 1.1\right)$ also for other size distributions, which are defined in section 3.1.3. We only remark that the bidisperse systems - equivalent to systems with uniform volume distribution - show some fine structure around the jumps, 
which we do not discuss here for the sake of brevity.

In Fig. 3.4, systems with uniform size distribution shown for different size ratios $\omega$. It can be seen how the ordering disappears as the size ratio increases.

In Fig. 3.5 bidisperse systems corresponding to uniform size distributions are shown for different size ratios, i.e., with (relatively) small size ratios just below the crystallization ratio, Fig. 3.5(a), just above, Fig. 3.5(b), and with (relatively) large size ratios just below and above partial (single species) crystallization in Figs. 3.5(c) and 3.5(d), respectively. Note that changing of the polydispersity from $10 \%$ (a) to $11 \%$ (b) changes the physical state of the system a lot, so that we can see the difference even by the eye, which is quite remarkable. This is consistent with results from Ref. [188], where for a binary mixture with a size ratio 1.111 and $n_{1}=0.5$ crystallization was almost totally suppressed.

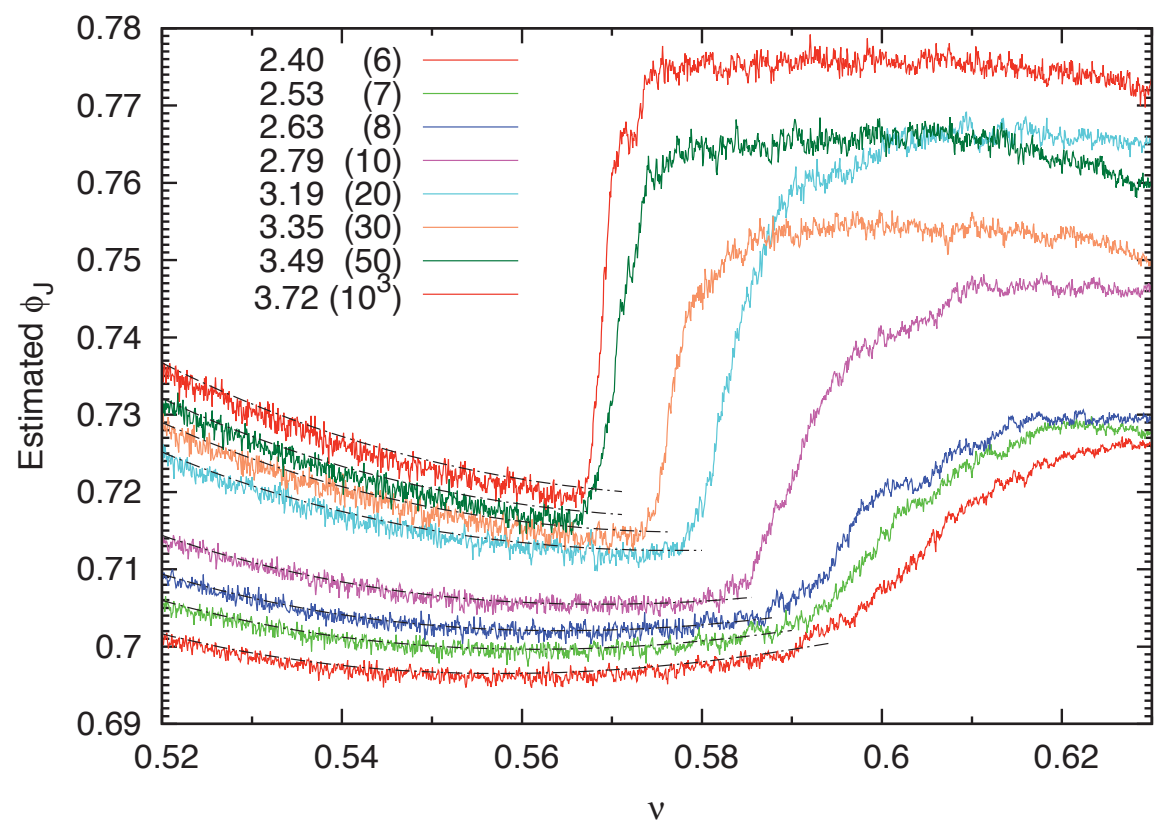

Figure 3.3: The estimated jamming density $\phi_{J}$ as a function of volume fraction $\nu$ for bidisperse systems corresponding to uniform size distribution. Increasing $\omega$ corresponds to bottom-to-top, with growth rate $\Gamma=16 \times 10^{-6}$. The (metastable) freezing density is decreasing with increasing size ratio. The BMCSL EOS are shown by dash-dotted lines. 

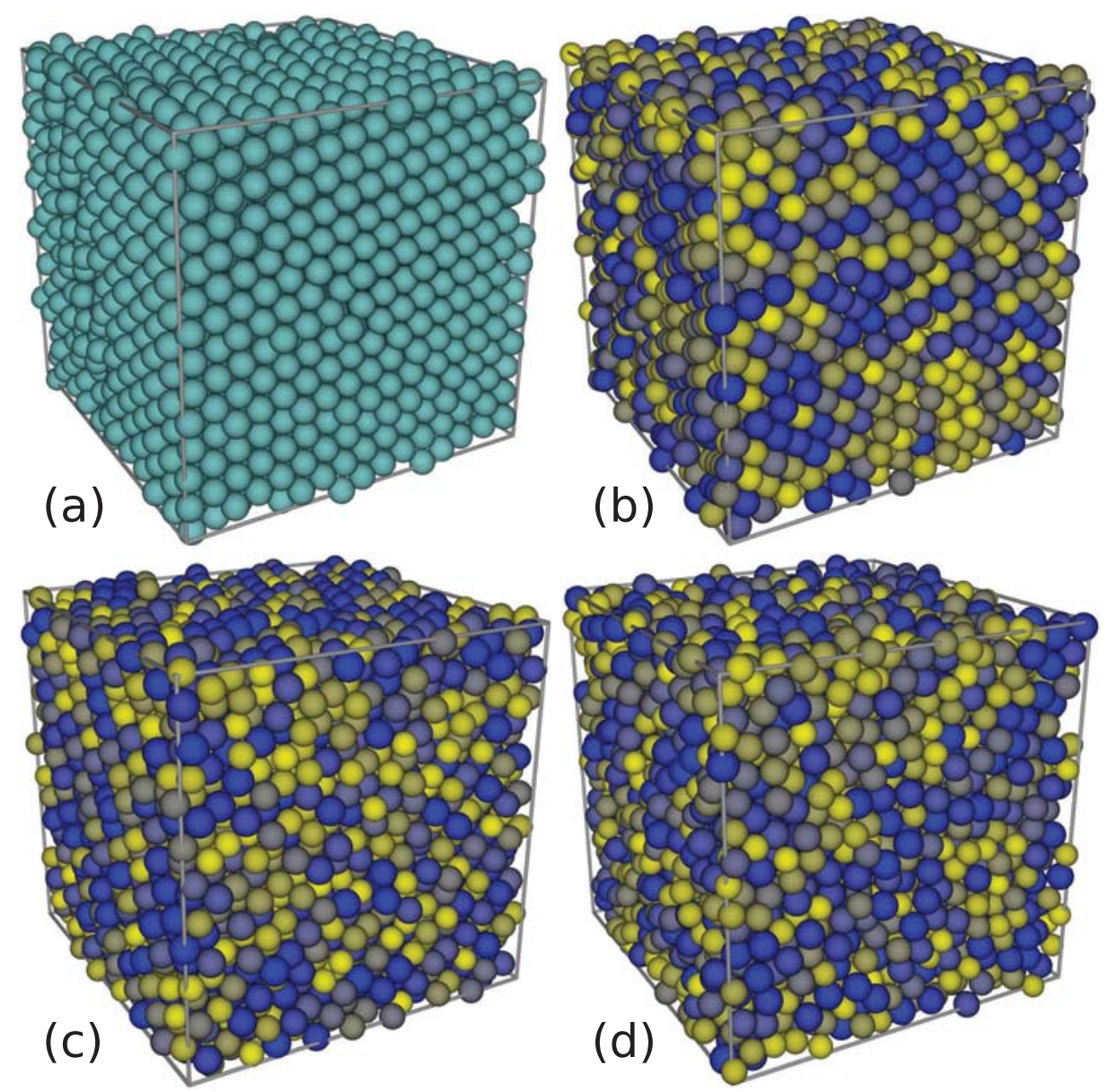

Figure 3.4: Particle systems with uniform size distribution with different size ratios at densities very close to jamming. Size ratios are $\omega=1$ (a), $\omega=1.12$ (b), $\omega=1.18$ (c), and $\omega=1.22$ (d). The order-disorder transition can be clearly seen as the size ratio increases. Color is by relative size, i.e., yellow (light) corresponds to small particles, and blue (dark) corresponds to big ones. The used growth rate to reach these configurations was $\Gamma=8 \times 10^{-6}$.

\section{Bidisperse versus polydisperse}

The aim of this section is to compare the equation of state of polydisperse systems and their bidisperse equivalents at volume fractions where fluid theory is not valid, i.e., in their "glassy" states or in coexistence of fluid and solid phases. Figure 3.6 shows $\phi_{J}$ as a function of volume fraction for a few systems with uniform size distribution and their bidisperse equivalents, 3.6(a), and for systems with uniform volume distribution and their bidisperse equivalents, 3.6(b). 

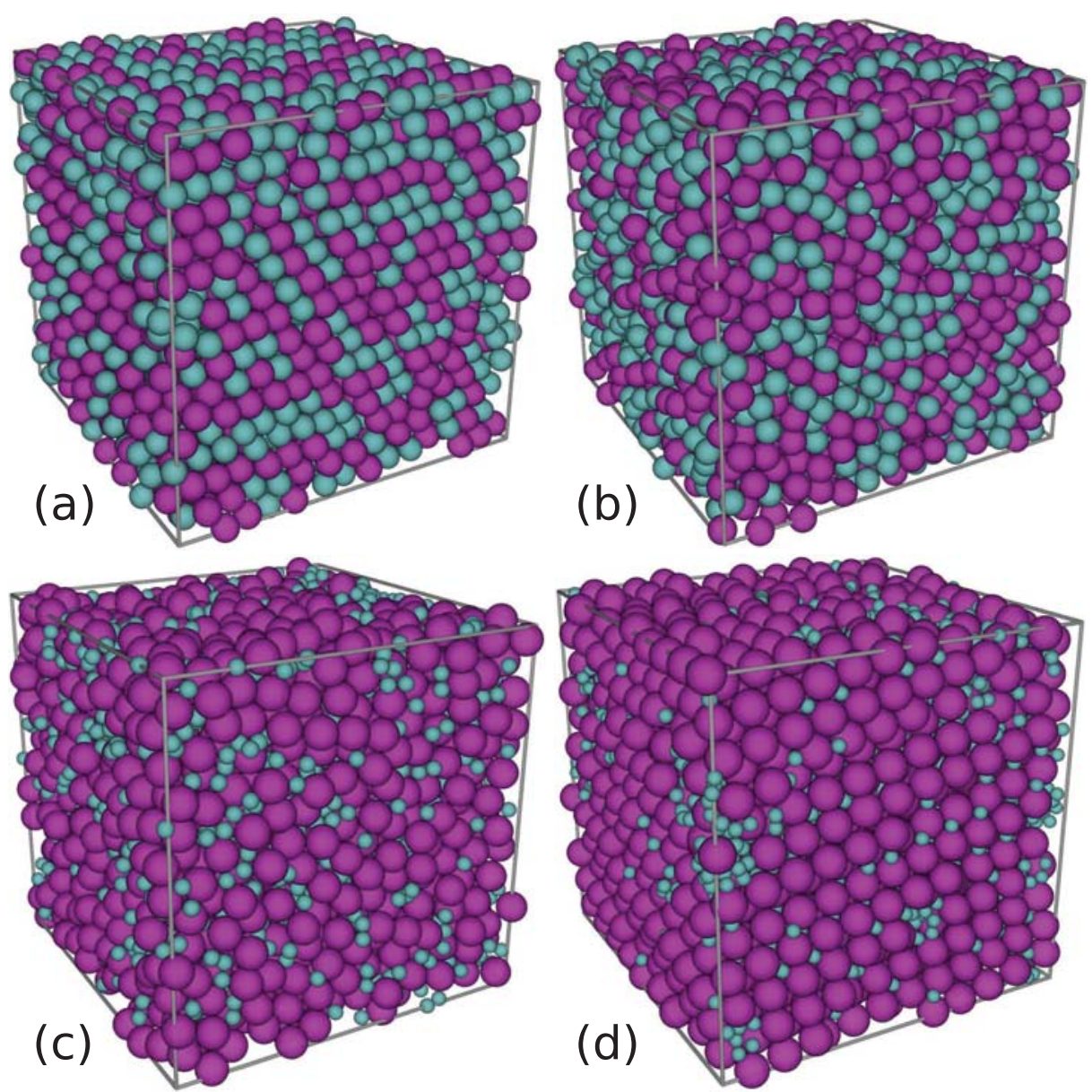

Figure 3.5: Particle systems with bidisperse size distribution corresponding to a uniform size distribution, i.e., $n_{1}=1 / 2$, with different size ratios at densities very close to jamming. Size ratios are $R^{\text {bi }} \approx 1.100(\omega=1.18)$ $(\mathrm{a}), R^{\mathrm{bi}} \approx 1.111(\omega=1.2)(\mathrm{b}), R^{\mathrm{bi}} \approx 2.060(\omega=4)(\mathrm{c})$, and $R^{\mathrm{bi}} \approx 2.404$ $(\omega=6)(d)$. The order-disorder transition can be clearly seen. Note that in (d) also some signs of segregation / clusterization of small particles can be seen, though investigation of this is beyond the scope of this paper.

While for small size ratios, $\omega \lesssim 1.2$, ordering / crystallization occurs, see Fig. 3.2(a), for moderate size ratios, $1.2 \lesssim \omega \lesssim 4$, the estimated jamming density of the considered polydisperse systems and their respective bidisperse equivalents are within 1\%, where the bidisperse data are slightly below. Thus, one can reduce mixtures to two-component (binary) systems, which have similar physics, even when the system densities are above the fluid-regime.

The equivalency of two- and many-component mixtures breaks down in the case of uniform size distribution as polydispersity (width) increases, e.g., 
for $\omega=6\left(R^{\mathrm{bi}} \approx 2.4, n_{1}^{\mathrm{bi}}=1 / 2\right)$, due to partial crystallization of the big particles in the bidisperse system, as it can also be seen in Fig. 3.5(d). This is consistent with results from Ref. [189], where the structure of binary hard sphere mixtures is studied in more detail. This kind of partial crystallization happens since the small particles fit into interstices of a crystal made of the large particles [24]. Such "colloidal alloy" structures have been observed both in naturally-occurring opals [190] and synthetic colloids [191,192].

In the case of bidisperse distributions corresponding to uniform volume distributions, we did not observe strong evidence of partial crystallization in the $1.18 \leq \omega \leq 10\left(1.10 \lesssim R^{\mathrm{bi}} \lesssim 3.89\right)$ range, even though a bit of restructuring for $\omega \geq 5$, see Fig. 3.6(b), seems to happen.

In the inset of Fig. 3.6(b) the zoomed data for $\omega=8$ are plotted, showing that the (final) jamming density can change (jump) also very close to the identity line $\phi_{J}=\nu$, indicating considerable re-structuring even very close to jamming.

In the following section we determine more precisely the range of size ratios for which systems show crystallization and/or partial single-species ordering.

\section{Towards the jamming density}

In this section, we investigate the maximum density $\nu_{\max }$ as a function of polydispersity. By maximum density we mean the density obtained after long-term (slow) compression, i.e., further compression for a time period about $4 \times 10^{5}$ events per particle does not increase the density, possibly due to numeric limits. The average compressibility factor of the final (jammed) systems is typically $Z \geq 10^{13}$.

In the amorphous regime $\phi_{J}$ decreases ( $Z$ increases) relative to the fluid prediction, due to excluded volume effects. In Figs. 3.2 and 3.6, ordering / crystallization was characterized by an increase of $\phi_{J}$ above the fluid prediction (i.e. a decrease of $Z$ below). Now, we also relate this to the change of behavior of $\nu_{\max }$, which is consistent with Ref. [162].

Figure 3.7 shows the maximum density $\nu_{\max }$ as a function of the inverse size ratio $\omega^{-1}$ for different size distributions and for different compression rates. The size ratio $\omega$ corresponds to polydisperse systems, while equivalent bidisperse ones are constructed using formulas (3.20) and (3.21). Firstly, we see that for small polydispersity $\omega^{-1} \gtrsim 0.85$, i.e., $\omega \leq 1.18\left(R^{\text {bi }} \lesssim 1.100\right)$, all the considered systems display (partial) crystallization or ordering effects, characterized by a jump (discontinuous increase) in $\nu_{\max }$ with $\omega^{-1}$. Note that the bidisperse systems corresponding to uniform volume distributions do not crystallize for $\omega=1.18$ (checked for three different runs), but for slightly smaller $\omega \leq 1.17\left(R^{\text {bi }} \lesssim 1.095\right)$. Partial crystallization of this type (small $\omega$ ) 

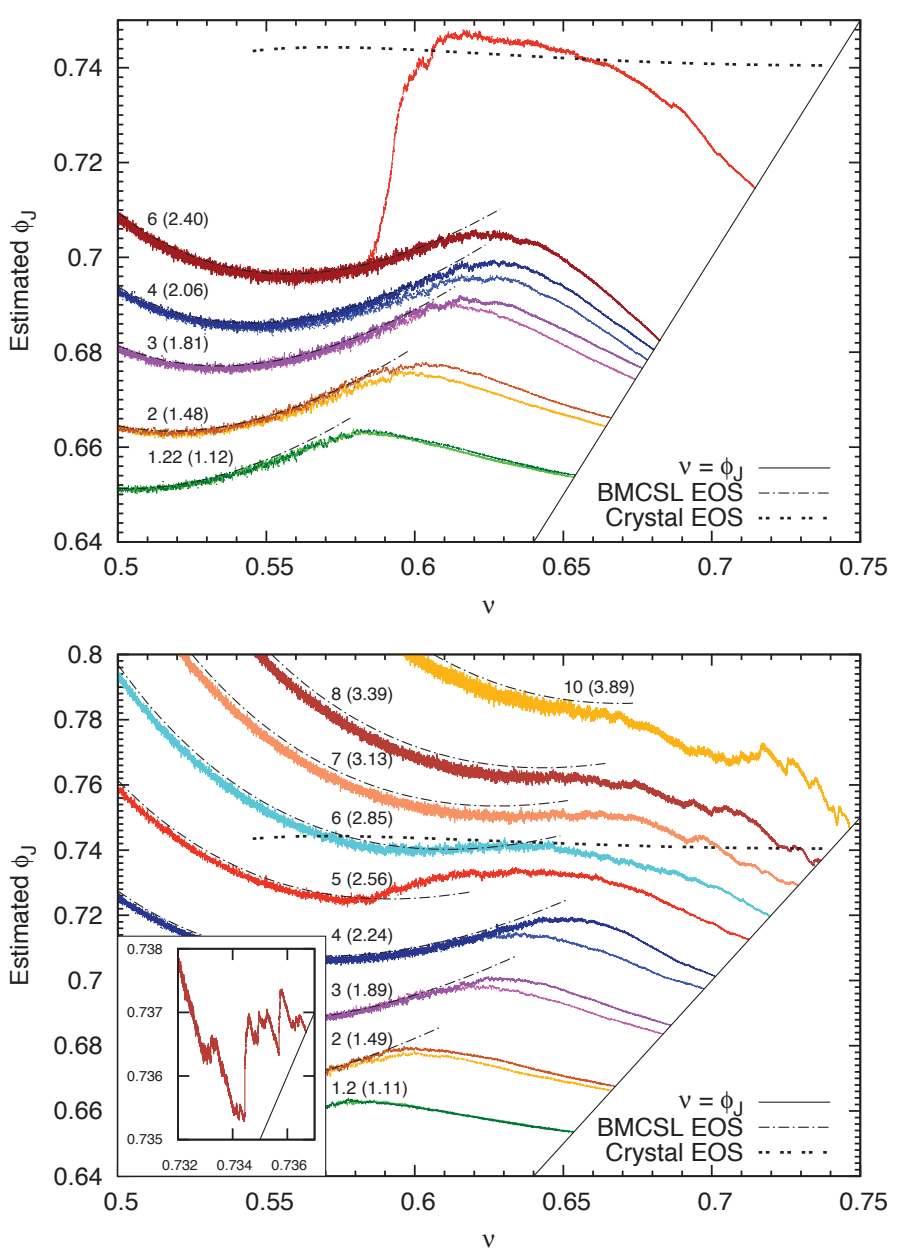

Figure 3.6: The estimated jamming density $\phi_{J}$ as a function of volume fraction $\nu$ for systems with (a, top) uniform size distribution and their bidisperse equivalents, using $\Gamma=8 \times 10^{-6}$, and for systems with (b, bottom) uniform volume distribution and their bidisperse equivalents, using $\Gamma=16 \times 10^{-6}$. Size ratios $\omega$ and $R^{\mathrm{bi}}$ are displayed in the inset, where the latter is given in brackets. Also plotted are the fluid theory (BMCSL EOS) and the approximation for the crystal phase [185]. Data for polydisperse systems in (b) are shown for $\omega \leq 4$. In the inset of (b) the zoomed data for $\omega=8$ are shown.

can be seen by the eye in Fig. 3.5(a).

In the $1.22 \leq \omega \leq 4$ range there is no (partial) crystallization observed for any of the considered systems. Furthermore, the maximum density of polydisperse systems is just slightly larger (within 0.5\%) than for their bidisperse equivalents, see the inset in Fig. 3.7(b). Note that in the case of uniform volume distributions, larger $\omega$ cannot be properly realized for too 


\section{Equation of state and jamming density}

small $N=4096$, so we do not show results for $\omega>5$.

For larger $\omega$, i.e., $\omega \geq 5$, bidisperse systems corresponding to uniform size distributions undergo partial crystallization of the large species $\left(R^{\mathrm{bi}} \gtrsim 2.25\right)$, which was discussed in the previous section.

As already evident in Fig. 3.6, the system with uniform volume distribution and their bidisperse equivalents pack "better" and reach much larger $\nu_{\max }$, see Fig. 3.7(b), when compared to 3.7(a), even without apparent partial (species) crystallization [194].

In contrast to their bidisperse counterparts, polydisperse systems with uniform size distribution do not show any signs of crystallization for $\omega \geq$ 1.22 , and the maximum density converges with increasing polydispersity to $\nu_{\max }^{\infty} \approx 0.6828 \pm 0.0004$, seemingly not changing much even for extremely wide distributions.

From our data, for uniform size distribution $\nu_{\max }$ can be fitted by a function of $\omega$ :

$$
\nu_{\max }(\omega)=\nu_{\max }^{\infty}-\left(\nu_{\max }^{\infty}-\phi_{\mathrm{RCP}}\right)\left(3 \omega^{-2}-2 \omega^{-3}\right),
$$

where the random close packing density $\phi_{\mathrm{RCP}}$ is taken to be 0.65 , as also predicted by cell theory [195]. The deviation is within $\pm 0.5 \%$ for all data $\omega \gtrsim 1.2$, see the inset in Fig. 3.7(b), and we enforced the derivative $\nu_{\max }^{\prime} \equiv 0$ for $\omega^{-1} \rightarrow 1$.

The maximum density of systems with uniform volume distribution and their bidisperse equivalents can be fitted by a function of $\omega$ in the range $1.2 \leq \omega \leq 10$ :

$$
\nu_{\max }(\omega)=\nu_{\max }^{\infty}-\left(\nu_{\max }^{\infty}-\phi_{\mathrm{RCP}}\right)\left(\omega^{-1}-\omega^{-2}+\omega^{-3}\right)\left(1-\log \omega^{-2}\right),
$$

where $\phi_{\mathrm{RCP}}=0.647$ and $\nu_{\max }^{\infty}=0.859$, with deviation within $\pm 1 \%$, see the inset in Fig. 3.7(b), and we enforced the derivative $\nu_{\max }^{\prime} \equiv 0$ for $\omega^{-1} \rightarrow 1$. The bidisperse equivalents deviate from Eq. (3.16) for $\omega>10$ due to increasing relative volume of the large spheres with increasing $\omega$ [163]. Remarkably, in this range Eq. (3.16), with swapped values of $\phi_{\mathrm{RCP}}$ and $\nu_{\max }^{\infty}$, fits the data well, as can be seen in Fig. 3.7(b). The two distinct regimes of $\nu_{\max }$ of bidisperse systems with moderate to large size ratios are predicted by a number of recently published theories [163-166]. A few checks of our bidisperse data with theoretical curves shown in Figure 6 in Ref. [163] show consistency. For a more comprehensive study of the densest binary packing of hard spheres see also Ref. [196].

The reported high-density limit for random close packings [197] is 3\% greater than that of Bernal packings [198] and still higher than our $\phi_{\mathrm{RCP}}$ values, and can be approached for extremely fast compression [66,162]. For estimates of $\phi_{\mathrm{RCP}}$ for random close packings of hard spheres see Ref. [199]. 
Note that the analytical expressions for $O_{1}$ and $O_{2}$ for two of the considered polydisperse size distributions shown in Appendix involve power laws and logarithmic functions as in Eqs. (3.15) and (3.16), respectively. This suggests that the jamming density in general could be expressed through $O_{1}$ and $O_{2}$, though we did not investigate this possibility in detail and leave it for further research.

\section{Different growth rates}

The effect of using different growth rates $\Gamma$ on the maximum density is shown in the inset of Fig. 3.7(a) for two size distributions. We observe that the maximum density slightly increases (within 1\%) with decreasing the growth rate. There are no signs of saturation for $\Gamma \rightarrow 0$, which indicates a quite interesting slow dynamics, leaving plenty of questions open for further research. The effect of using various expansion rates is more comprehensively studied in Refs. $[66,67,161,162,200]$, so that we do not display more of our data. We did not study extremely slow rates and rate-dependence systematically. We only note that different growth rates can lead to different metastable freezing points [67].

\section{Super dense limit}

In this section we show that sufficiently close to the jamming density, the compressibility factor is independent of the size distribution and depends on only one parameter, the jamming density itself, complementing an earlier study of monodisperse systems [181]. Figure 3.8 shows the compressibility factor scaled by equation (3.14) for systems with uniform size distributions.

The jamming densities $\phi_{\mathrm{J}}$ used in Fig. 3.8 are determined using Eq. (3.14) by mapping the diverging compressibility factor to finite values, as suggested by Torquato et al. $[181,201]$. It must be noted that all $\phi_{\mathrm{J}}$ differ from $\nu_{\max }$ obtained in simulations by at most $10^{-13}$, which confirms that we are close to the infinite pressure limit. We see from Fig. 3.8 perfect agreement with the free volume theory for all considered systems at the densities $\left(\phi_{\mathrm{J}}-\nu\right) \lesssim 10^{-8}$. Only one free parameter is required for the free volume theory, $\phi_{\mathrm{J}}$, even though in other studies two fitting parameters are used $[159,162]$. In order to capture the deviation of the compressibility factor from the free volume theory as the density difference increases, theories which take into account the geometry and structure of the free volume should be developed. The point where data collapse with the free-volume theory is the point where cages are established and do not change any more [202].

It must be noted that the results of Fig. 3.8 are obtained using numerical double type ( 8 bytes). We used also long double type (16 bytes) for a few 
simulations and (while the data in Fig. 3.8 did not change) could reach perfect agreement with the free-volume theory Eq. (3.14), using raw data (without $\phi_{\mathrm{J}}$ mapping), even for $10^{-15} \lesssim\left(\nu_{\max }-\nu\right) \lesssim 10^{-8}$, i.e., over seven orders of magnitude, which is quite remarkable.

Finally, we remark that even though the free volume theory presented in Eq. (3.14) is designed for monodisperse systems, we observe a very good agreement for polydisperse systems as well (see Fig. 3.8). We also confirmed Eq. (3.14) for two-dimensional systems, and for systems with uniform volume and bidisperse size distributions (data not shown), and for different compression rates.

\subsubsection{Summary and Conclusions}

In this study we have shown analytically that for any given polydisperse system an equivalent bidisperse system can be constructed with the same equation of state in the fluid regime, using Eqs. (3.20) and (3.21). In order to confirm this result simulation data of the measured compressibility factor are compared to previously found equations of state and agreement was found with less than $1 \%$ deviation. Based on the simulation data a slightly more accurate equation of state (3.13) was proposed for the fluid regime.

Surprisingly, for densities higher than the freezing (or glass transition) density, beyond the fluid regime, our simulations show that a polydisperse system and its bidisperse equivalent have similar equations of state, i.e., the estimated jamming density is within $1 \%$, as long as the bidisperse systems do not show either (partial) crystallization or (theoretically predicted [163]) change in maximum-density-behavior due to the overwhelming presence of the major species.

We have observed three distinct types of liquid-solid organization / behavior in the bidisperse systems with equal number of small and big particles, depending on the size ratio $R^{\mathrm{bi}}$. First, for $R^{\mathrm{bi}} \lesssim 1.1$ the crystalline phase formed at high densities consists of a crystal made of both small and big particles; second, in the range $1.1 \lesssim R^{\mathrm{bi}} \leq 2.06$ we do not observe any signs of ordering effects, i.e., an amorphous solid is formed; finally, at moderate to large size ratios $R^{\text {bi }} \gtrsim 2.40$, we observe an ordered structure that consists of a crystal of predominately large particles. We confirm this using two different criteria to quantify partial crystallization, i.e., the compressibility factor and the jamming density. Note that above results are obtained using (relatively) slow density growth rates, while for extremely fast compression it is possible to avoid partial crystallization. For the polydisperse systems tested, we find partial crystallization only for (relatively) low size ratios $\omega$, i.e., $\omega \lesssim 1.2$, but not for larger $\omega$.

Sufficiently close to the jamming density the compressibility factor is in- 
dependent of both size distribution and compression rate and depends only on one free parameter, the jamming density itself. The free volume equation of state (3.14) is in very good agreement with our simulation results for all considered systems in two- and three-dimensions. We provide analytical (empirical fits) equations for the jamming density as function of the extreme size ratio for uniform size and uniform volume radii distributions to be confronted with theory and experiments.

The result with potential for applications is the fact that polydisperse systems can be modeled [203] by their bidisperse equivalents and their jamming (packing) density can be predicted from the moments $O_{1}$ and $O_{2}$. Furthermore, the results of this study can be used for better understanding the equation of state for mixtures of fluids and thus, to predict mixing / segregation. Finally, our predictions concerning the size ratios for which crystallization happens can be useful for experiments on colloids and granular media, to either avoid or establish ordering effects.

We would like to stress that this is a preliminary study that considers mostly relatively slow growth rates for a few size distribution function shapes. Whether our predictions also hold for very differently shaped and much wider size distribution functions is open as well as questions about much slower or faster growth rates.

In a future study we will introduce an equivalent tridisperse system and show that with this it is possible to suppress partial crystallization of binary systems also for large size ratios. Comparison with experiment is another subject of future research. 

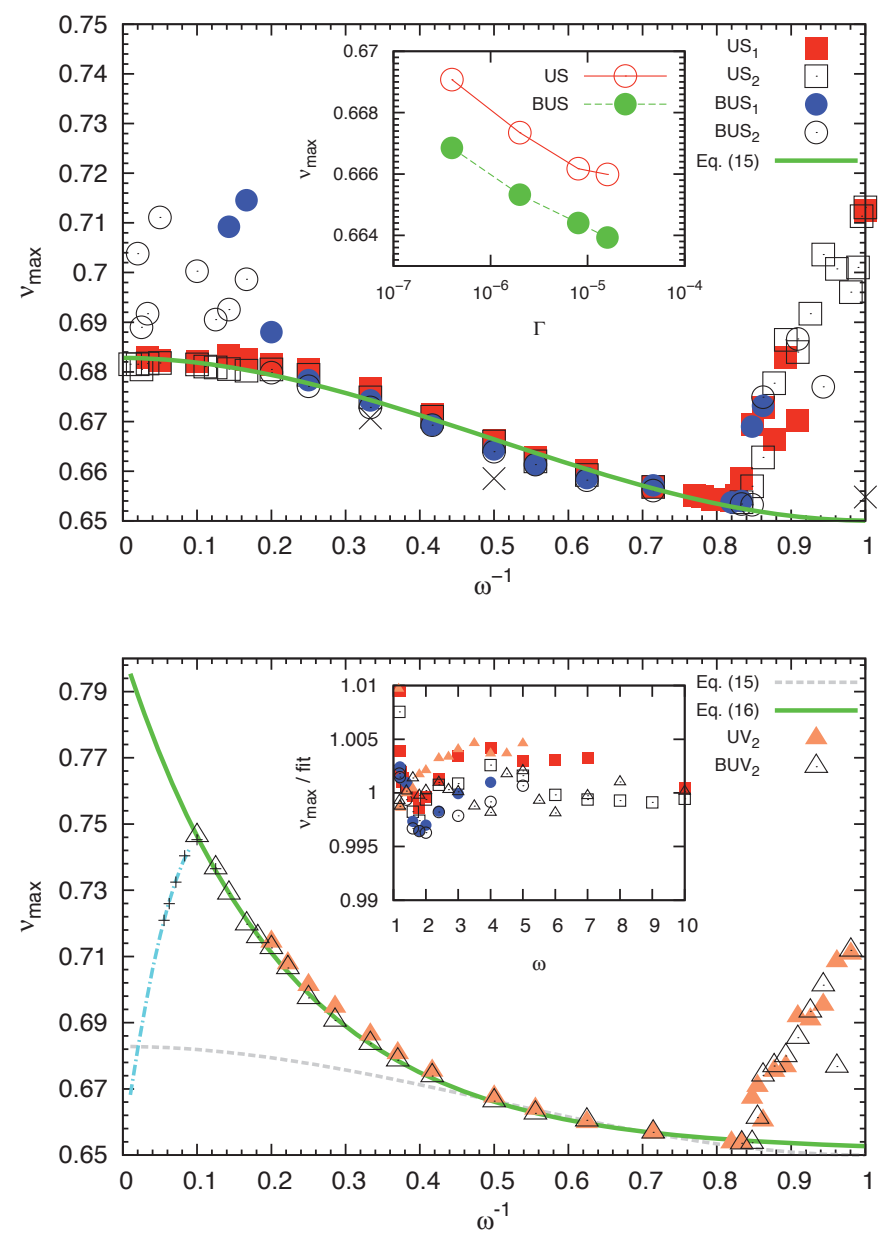

Figure 3.7: The maximum density $\nu_{\max }$ as a function of the inverse size ratio $\omega^{-1}$ for different size distributions and for different compression rates, in the inset subscript 1 corresponds to the reference growth rate $\Gamma=8 \times 10^{-6}$, and subscript 2 corresponds to $\Gamma=16 \times 10^{-6}$. Size distributions are (a, top) uniform size distribution (US) and their bidisperse equivalents (BUS), or (b, bottom) uniform volume (UV) and their bidisperse equivalents (BUV). The size ratio $\omega$ corresponds to the polydisperse systems, while bidisperse ones are constructed using 3.1.4. Results for BUV systems with $N=8192$, using $\Gamma=16 \times 10^{-5}$, are shown as pluses in (b), which are fitted for $\omega \geq$ 10 by Eq. (3.16) with swapped values of $\phi_{\mathrm{RCP}}$ and $\nu_{\max }^{\infty}$ (dash-dotted line). The left most point $(+, \omega=30)$ is higher than expected because of partial crystallization, setting in also here at very large $\omega$. Results from Ref. [193] obtained by compression of soft frictionless particles with a uniform size distribution are shown as crosses in (a). In the inset of (a), $\nu_{\max }$ is plotted for different growth rates $\Gamma$ for the uniform size distribution $(\omega=2)$ and their bidisperse equivalent $\left(R^{\mathrm{bi}} \approx 1.48\right)$. In the inset of $(\mathrm{b})$, the deviation of data from the fits is shown with corresponding symbols. 


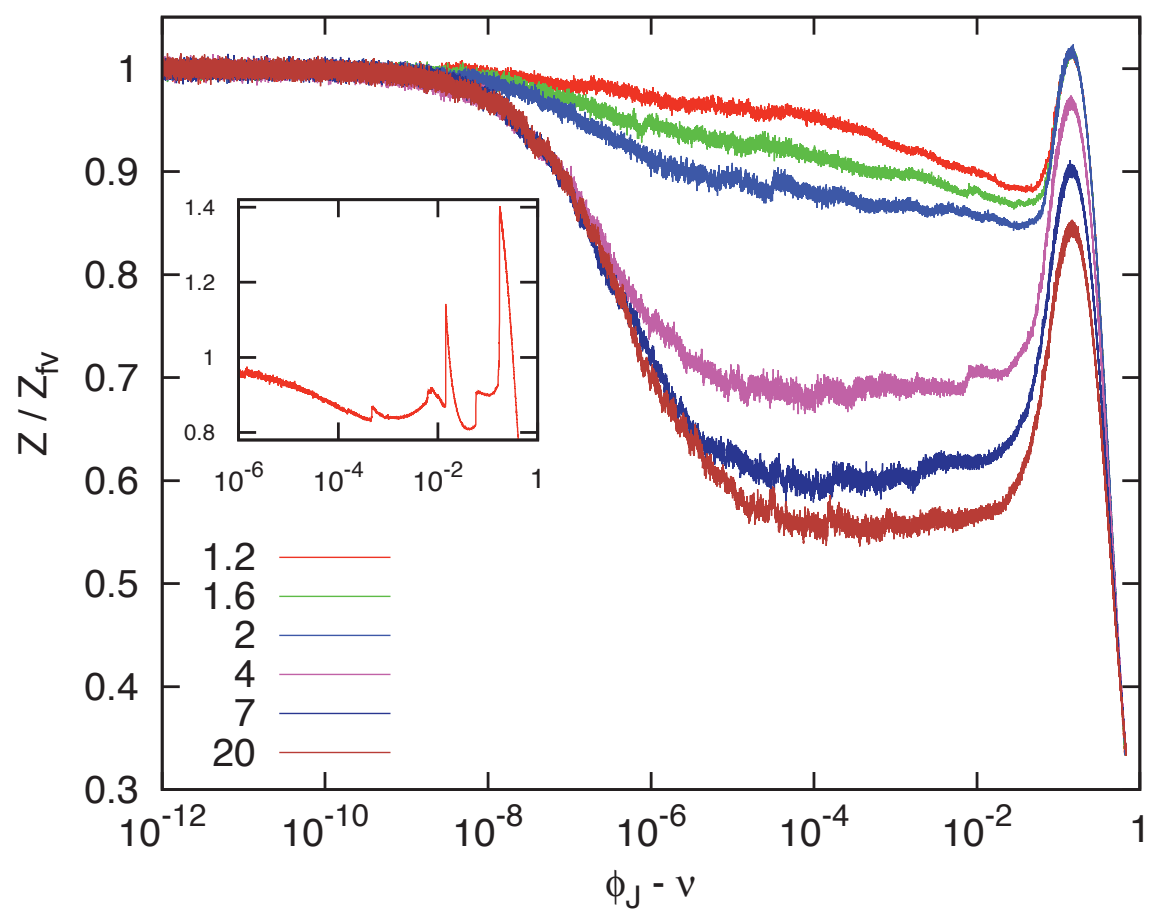

Figure 3.8: The compressibility factor scaled by the free volume equation of state (3.14) in the limit of diverging pressure for systems with uniform size distribution for different size ratios, increasing $\omega$ corresponds to topto-bottom, with growth rate $\Gamma=16 \times 10^{-6}$. When partial crystallization happens we see some fine structures as shown in the inset for the monodisperse system $(\omega=1)$. 


\section{Appendix}

\section{Derivation of the effective correlation function}

In order to express the compressibility factor in the 3D mixtures, using dimensionless moments, plug Eqs. (3.2), (3.3) and (3.4) into the expression in brackets of Eq. (3.5):

$$
\begin{aligned}
& g_{11} a_{11}^{3} n_{1}^{2}+2 g_{12} a_{12}^{3} n_{1} n_{2}+g_{22} a_{22}^{3} n_{2}^{2} \\
& \quad \equiv \frac{1}{1-\nu}\left(a_{11}^{3} n_{1}^{2}+2 a_{12}^{3} n_{1} n_{2}+a_{22}^{3} n_{2}^{2}\right) \\
& \quad+\frac{3 \nu \frac{A_{2}}{A_{3}}}{2(1-\nu)^{2}}\left(a_{11}^{3} n_{1}^{2}+\frac{4}{1+R} a_{12}^{3} n_{1} n_{2}+\frac{1}{R} a_{22}^{3} n_{2}^{2}\right) \\
& \quad+\frac{\left(\nu \frac{A_{2}}{A_{3}}\right)^{2}}{2(1-\nu)^{3}}\left(a_{11}^{3} n_{1}^{2}+\frac{8}{(1+R)^{2}} a_{12}^{3} n_{1} n_{2}+\frac{1}{R^{2}} a_{22}^{3} n_{2}^{2}\right) \\
& \quad \equiv \frac{6\langle a\rangle\left\langle a^{2}\right\rangle+2\left\langle a^{3}\right\rangle}{1-\nu}+\frac{6 \nu\left(\langle a\rangle\left\langle a^{2}\right\rangle\left\langle a^{3}\right\rangle+\left\langle a^{2}\right\rangle^{3}\right)}{(1-\nu)^{2}\left\langle a^{3}\right\rangle} \\
& \quad+\frac{4 \nu^{2}\left\langle a^{2}\right\rangle^{3}}{(1-\nu)^{3}\left\langle a^{3}\right\rangle} .
\end{aligned}
$$

Using the expression (3.17), and inserting Eqs. (3.6), one obtains:

$$
\begin{aligned}
Z-1 & =(1+r) \frac{\nu}{4\left\langle a^{3}\right\rangle}\left(g_{11} a_{11}^{3} n_{1}^{2}+2 g_{12} a_{12}^{3} n_{1} n_{2}+g_{22} a_{22}^{3} n_{2}^{2}\right) \\
& =(1+r) 2 \nu \frac{(1-\nu)^{2}+3 O_{1}(1-\nu)+O_{2}(3-\nu) \nu}{4(1-\nu)^{3}} \\
& =(1+r) 2 \nu g_{\mathrm{eff}}(\nu),
\end{aligned}
$$

where $r$ is the restitution coefficient.

\section{Bounds of dimensionless moments}

In order to show that $O_{1} \leq 1$, find the extrema of $g\left(\left\{a_{i}\right\}\right)=N O_{1}=$ $\sum a_{i} \sum a_{i}^{2} / \sum a_{i}^{3}, i \in[1, N]$ on the interval $a_{i} \in(0, \infty)$. For this solve the system of $k=1, \ldots, N$ equations:

$$
\frac{\partial g\left(\left\{a_{i}\right\}\right)}{\partial a_{k}}=\left[\frac{2 a_{k} \sum a_{i}+\sum a_{i}^{2}}{\sum a_{i}^{3}}-\frac{3 a_{k}^{2} \sum a_{i} \sum a_{i}^{2}}{\left(\sum a_{i}^{3}\right)^{2}}\right]=0 .
$$

The system (3.19) has one (trivial) solution $a_{i}=a=$ const, which corresponds to the monodisperse case. By looking at the sign of the second derivative of $g\left(a_{i}=a\right)$ it can be checked that this extremum is the local maximum $g^{\max }=N$. Therefore the local maximum of $O_{1}$ is equal to unity.

By analogy, considering the extrema of the function $f\left(a_{i}\right)=O_{2} / O_{1}$, it 
can be shown that $O_{2} \leq O_{1}$.

Finally, to show that $O_{1}^{2} \leq O_{2}$, note that $O_{1}^{2} / O_{2}=A$, where $A=$ $\langle a\rangle^{2} /\left\langle a^{2}\right\rangle \leq 1$. This yields $O_{1}^{2} \leq O_{2}$, where equality is reached only in the monodisperse case $\left(O_{1}=O_{2}=1\right)$.

\section{Equivalent bidisperse systems}

In order to find a bidisperse system that has the same dimensionless moments $O_{1}$ and $O_{2}$ as a given polydisperse one, solve analytically the system of equations (3.6) in terms of the variables $n_{1}$ and $R$. This yields a unique solution $\left(O_{1} \neq O_{2}\right)$ :

$$
\begin{gathered}
n_{1}^{\mathrm{bi}}=\frac{1}{2}+\frac{3 O_{1} O_{2}-O_{2}-2 O_{1}^{3}}{2 \lambda}, \\
R^{\mathrm{bi}}=\frac{2 O_{1}^{3}+2 O_{2}^{2}+O_{2}-4 O_{1} O_{2}-O_{1}^{2} O_{2}+\left(1-O_{1}\right) \lambda}{2\left(O_{2}-O_{1}\right)\left(O_{1}^{2}-O_{2}\right)},
\end{gathered}
$$

where $\lambda=\sqrt{O_{2}} \sqrt{4 O_{1}^{3}+O_{2}-3 O_{1}\left(2+O_{1}\right) O_{2}+4 O_{2}^{2}}$ and $n_{1}^{\text {bi }}$ corresponds here to the number fraction of large particles, i.e., $R^{\text {bi }} \geq 1$. Inserting these values into Eq. (3.8) leads also to the same compressibility factor $Z^{\mathrm{bi}}\left(n_{1}^{\mathrm{bi}}, R^{\mathrm{bi}}\right)=Z^{\text {poly }}\left(O_{1}, O_{2}\right)$.

\section{Growth rate}

In order to maintain the size distribution during the growing process, the radius $a_{i}$ of the particle $i$ changes with time as:

$$
\frac{d a_{i}}{d t}=\Gamma \frac{a_{i}}{a_{\max }(t)}
$$

where $a_{\max }(t)$ is the radius of the largest particle (which depends on time) and $\Gamma$ is the growth rate with units of velocity. In this convention, we have $a_{\max }(t)=\Gamma t$ and $a_{\min }(t)=\Gamma \omega^{-1} t$, where $a_{\min }(t)$ is the radius of the smallest particle and $\omega$ is constant, as desired. This ensures that the relative distribution of radii and thus sphere volumes around the mean is constant over time, but the mean sphere volume increases uniformly with time.

\section{Dimensionless moments for different size distributions}

Assume a polydisperse distribution of particle radii with probability $f(a) d a$ to find the radius $a$ between radii $a$ and $a+d a$, and with $\int_{0}^{\infty} f(a) d a=$ 1. Using the definition of the $k$-th moment of the size distribution $f(a)$, $\left\langle a^{k}\right\rangle \equiv \int a^{k} f(a) d a$, it is straightforward to calculate dimensionless moments 
$O_{1}=\langle a\rangle\left\langle a^{2}\right\rangle /\left\langle a^{3}\right\rangle$ and $O_{2}=\left\langle a^{2}\right\rangle^{3} /\left\langle a^{3}\right\rangle^{2}$ for any given polydisperse size distribution.

For the uniform size distribution, with $\omega=a_{\max } / a_{\min }$, we obtain:

$$
O_{1}=1-\frac{2}{3} \frac{\omega_{0}^{2}}{1+\omega_{0}^{2}}, O_{2}=\frac{1}{27} \frac{\left(3+\omega_{0}^{2}\right)^{3}}{\left(1+\omega_{0}^{2}\right)^{2}}
$$

where $\omega_{0}=(\omega-1) /(\omega+1)$, and $2 \omega_{0}\langle a\rangle$ is the width of size distribution function $f(a)$. It must be noted, that plugging Eq. (3.23) into Eq. (3.20) yields $n_{1}^{\text {bi }}=1 / 2$. Therefore, a bidisperse system which is equivalent to a polydisperse system with uniform size distribution has the same number of small and large particles for any $\omega$.

For the uniform volume distribution, we have $(\omega>1)$ :

$$
O_{1}=\frac{2 \omega}{\omega^{2}-1} \ln \omega, O_{2}=\frac{2 \omega^{2}}{(\omega-1)^{3}(\omega+1)} \ln ^{3} \omega
$$

For examples of numerical values see Table 3.1.

The moments of the log-normal distribution (not used in our present simulations) can be computed from the moment generating function of the normal distribution [204]. If $X$ has the log-normal distribution with parameters $\mu$ and $\sigma$ then $\mathbb{E}\left(X^{n}\right)=\exp \left[n \mu+(1 / 2) n^{2} \sigma^{2}\right], n \in \mathbb{N}$. Therefore, we obtain:

$$
O_{1}=\exp \left(-2 \sigma^{2}\right), O_{2}=\exp \left(-3 \sigma^{2}\right) .
$$

In a number of studies $[28,35,184]$ the magnitude of the spread of sphere radii is conveniently characterized by the parameter $\delta$, which is often also referred to as polydispersity and measures the standard deviation of the radii distribution normalized by its mean: $\delta=\left(\left\langle a^{2}\right\rangle-\langle a\rangle^{2}\right)^{1 / 2} /\langle a\rangle$. Therefore we also provide an expression for $\delta$ as function of $O_{1}$ and $O_{2}: \delta=\left(O_{2} / O_{1}^{2}-1\right)^{1 / 2}$, which is directly related to the parameter $A$ used in Ref. [159], $A=1 /\left(\delta^{2}+1\right)$. 


\begin{tabular}{|c|c|c|c|c|c|c|c|}
\hline Distr. & Figs. & $\omega$ & $O_{1}$ & $O_{2}$ & $R^{\text {bi }}$ & $n_{1}^{\text {bi }}$ & $\delta$ \\
\hline US & $3.1,3.2,3.3$, & 1.0 & 1.0 & 1.0 & 1.0 & & 0 \\
& $3.4,3.5,3.6(\mathrm{a})$, & 1.2 & 0.995 & 0.992 & 1.11 & & 0.05 \\
& $3.7(\mathrm{a}), 3.8$ & 1.4 & 0.982 & 0.973 & 1.21 & & 0.10 \\
& & 2 & 0.933 & 0.903 & 1.48 & & 0.19 \\
& & 4 & 0.824 & 0.760 & 2.06 & & 0.35 \\
& & 5 & 0.795 & 0.725 & 2.25 & $1 / 2$ & 0.38 \\
& & 10 & 0.733 & 0.657 & 2.79 & & 0.47 \\
& & 20 & 0.700 & 0.624 & 3.19 & & 0.52 \\
& & 50 & 0.680 & 0.605 & 3.49 & & 0.55 \\
& & 100 & 0.673 & 0.599 & 3.61 & & 0.57 \\
\hline UV & $3.1(\mathrm{~d}), 3.6(\mathrm{~b})$, & 1.0 & $2 / 3$ & $16 / 27$ & $2+\sqrt{3}$ & & $\sqrt{3} / 3$ \\
& $3.7(\mathrm{~b})$ & 1.2 & 0.994 & 0.992 & 1.11 & 0.453 & 0.05 \\
& & 1.4 & 0.981 & 0.972 & 1.21 & 0.413 & 0.10 \\
& & 2 & 0.924 & 0.888 & 1.49 & 0.327 & 0.20 \\
& & 4 & 0.739 & 0.632 & 2.24 & 0.190 & 0.39 \\
& & 5 & 0.671 & 0.543 & 2.56 & 0.156 & 0.46 \\
& & 7 & 0.568 & 0.418 & 3.13 & 0.114 & 0.55 \\
& & 8 & 0.528 & 0.373 & 3.40 & 0.100 & 0.58 \\
& & 10 & 0.465 & 0.304 & 3.90 & 0.080 & 0.64 \\
& & 100 & 0.092 & 0.020 & 17.83 & 0.006 & 1.16 \\
& & $\infty$ & 0 & 0 & - & - & - \\
\hline
\end{tabular}

Table 3.1: Given are size ratios $\omega$ and dimensionless moments $O_{1}$ and $O_{2}$ for a few polydisperse systems with uniform size (US) and uniform volume (UV) radii distributions and $R^{\mathrm{bi}}$ with $n_{1}^{\mathrm{bi}}$ for their bidisperse equivalents. 


\subsection{Poly- versus tri-disperse systems ${ }^{\dagger}$}

How many state-variables are needed to predict the equation of state and the jamming density of polydisperse mixtures in glassy, non-equilibrium compressed states? We propose to define equivalent and maximally equivalent systems as those that match three and five moments of a given polydisperse size distribution, respectively. Fluids can be represented well by an equivalent system with only $s=2$ components (bidisperse). As little as $s=3$ components (tridisperse) are enough to achieve a maximally equivalent system. Those match macroscopic properties in glassy states, but also the volume fraction of rattlers, suggesting strong microstructural equivalency too. For many soft and granular systems, tridisperse, maximally equivalent systems allow for a closed analytical treatment and well-controlled industrial applications, while our proposal waits for experimental validation.

The hard-sphere model is one of the simplest representations of soft condensed matter systems where strong repulsions dominate the weak, or negligible attractive forces. It can be applied with some success for studying disorder-order transitions, the glass transition, colloids, granular materials, amorphous metals, and phase transitions or nucleation in simple gases and liquids [2,138-140]. Despite its simplicity, more complicated (soft) interactions of spheres can be approximated with the hard-sphere model $[205,206]$.

In many experimental or industrially relevant circumstances, the particular components (spheres) of a system are not uniform in size but rather display some distribution of sizes or "polydispersity" [164]. Interesting phenomena arise in the presence of a wide polydispersity [25, 34, 207], but due to the wide size distribution across many scales, it is difficult to provide simple accurate statistical models [208,209] for such systems. In many fluidbased theories, for example, Percus-Yevick integral equation theory [210,211], scaled-particle theory [55], Boublík, Mansoori, Carnahan, Starling, and Leland (BMCSL) equation of state (EOS) [57,58], Rosenfeld's fundamental measure theory [212], A. Santos' approaches [59-61], and others [49, 52, 213], it is assumed that the dependence of the polydisperse pressure of $N$ hard spheres on the $N$ degrees of freedom (volume fraction plus $N-1$ size ratios) can be encapsulated into the dependence of only three parameters, i.e., the volume fraction and the second and third scaled moments (divided by the first moment, with appropriate power). In this case, the equivalent map-

\footnotetext{
${ }^{\dagger}$ Based on V. Ogarko and S. Luding, "Prediction of polydisperse hard-sphere mixture behavior using tridisperse systems," Soft Matter, vol. 9, no. 40, pp. 9530-9534, 2013.
} 
ping polydisperse-to-bidisperse is enough, because in order to match the first three moments of any radii distribution requires only two differently sized components. At the same time, the two-species kinetic theory is particularly simple, since two coupled equations for each species can be written down explicitly, whereas the multi-species theory is much less convenient.

A bidisperse equivalent mapping, while being very useful over a wide range of fluid volume fractions, $\nu_{f} \lesssim 0.56$, has limitations for extremely large densities in glassy, metastable states near the jamming transition. Equivalent bidisperse systems show (partial) crystallization for densities around $\nu_{f}$ already and, in general, do not perform well for $\nu>\nu_{f}$, due to the overwhelming presence of one species [27]. The open question is whether another mapping polydisperse-to-finite-number of components works, starting from as little as a three-component mixture.

We consider $s$-component mixtures of $N_{i}$ hard spheres of radius $a_{i}$ enclosed in a volume $V$, with $i=1, \ldots, s$. In the case of a polydisperse size distribution, $s=N$ and each sampled $a_{i}$ can be different from the others. The composition of such mixtures is quantified by the radii $a_{i}$ and the partial volume fractions, $\nu_{i}=(4 / 3) \pi N_{i} a_{i}^{3} / V$, i.e., one has $2 s$ degrees of freedom (dof). The total volume fraction $\nu=\sum_{1}^{s} \nu_{i}$ and the arbitrary unit of length, leave $2 s-2$ independent dof. We define equivalent systems as those that have the same first two scaled moments, $k=2,3$, with $M_{k}=\left\langle a^{k}\right\rangle /\langle a\rangle^{k}$, with moments, $\left\langle a^{k}\right\rangle=\int a^{k} f(a) \mathrm{d} a$, of their normalized size distribution functions $f(a)$. Furthermore, maximally equivalent systems are those that have the same first four scaled moments, $k=2-5$, of their size distribution functions. Without loss of generality, it can be more convenient ${ }^{\dagger}$, [52] to use central moments $\mu_{k}=\left\langle(a-\langle a\rangle)^{k}\right\rangle$.

The larger $s$, the more moments of the size distribution can be adjusted, and due to more efficient packing it is possible to reach considerably higher jamming densities as compared to mono- or bidisperse cases. Perfectly spacefilling (e.g., Apollonian) packings are beyond the reach of tridisperse packings [214], as well as infinitely slow routes to equilibrium states ${ }^{\ddagger}$, due to finite simulation times.

We perform Event-Driven molecular dynamics simulations using systems of hard spheres with periodic boundary conditions. Starting from zero volume fraction we compress the system towards a jammed state using a modification of the Lubachevsky-Stillinger algorithm [81,134], which allows the diameter of the particles to grow linearly in time with a dimensionless rate $\Gamma \S$, while the kinetic energy, $E$, is kept constant using a re-scaling thermostat procedure [27]. An alternative growth method would decrease $\Gamma$ with in-

\footnotetext{
₹Thermodynamically, the polydisperse system fractionates in phases separated according to the particle size, but this eutectic freezing-transition is kinetically suppressed and practically unreachable [215].
} 
creasing density [216]. However, the used compaction process is well-defined nevertheless, and the resultant (non-equilibrium) states too - as confirmed by some different initial configurations - albeit subject to statistical fluctuation and ensemble averaging for small finite systems. The growth rate used in most simulations is slow, $\Gamma=16 \times 10^{-6}$, if not explicitly stated otherwise. A few runs with ten times smaller $\Gamma$ are consistent. The compressibility factor $Z \equiv p V / N k_{B} T$ (dimensionless combination of pressure $p$ and kinetic temperature $k_{B} T=2 E / 3 N$ ), is calculated from the total exchanged momentum in all interparticle collisions during a short time period. The following types of particle size distributions are used: (i) uniform size (rectangular); (ii) uniform volume, i.e., the total volume occupied with those particles with radii between $a_{1}$ and $a_{1}+d a$ is equal to the total volume occupied by particles with radii between $a_{2}$ and $a_{2}+d a$, etc; (iii) tridisperse systems, equivalent to the aforementioned polydisperse systems. The considered polydisperse distributions are characterized by their width, i.e., the ratio $\omega$ between the maximum and the minimum particle radius. (For details see section 3.3, where the log-normal distribution is also discussed.)

Our simulations confirm that in the fluid regime, i.e., at volume fractions $\nu<0.54$, the agreement in the compressibility factor $Z$ between all considered systems and the BMCSL equation of state is better than $1 \%$ (data not shown) [27]. When the density is further increased, the compressibility factor deviates from the fluid theory prediction, that involves a maximum volume fraction of unity due to the density expansion involved, while the maximum solid volume fraction has to be lower due to excluded volume. $Z$ increases very rapidly at densities higher than the freezing point, so that we instead plot the estimated jamming density $\phi_{J}^{\text {est }}(\nu)=\nu /[1-3 / Z(\nu)][27]$, since it is limited, i.e., $\phi_{J}^{\text {est }}(\nu)=\nu$ for $Z(\nu) \rightarrow \infty$. Figure 3.9(a) shows $\phi_{J}^{\text {est }}$ as a function of volume fraction for a few systems with uniform size distribution (dark curves, e.g., blue) and their tridisperse, maximally equivalent counterparts (light curves, e.g., cyan), i.e., with matched first four scaled moments. While for small size ratios, $\omega<1.2$, ordering/crystallization is expected ${ }^{\dagger}$, [27], for $1.2 \leq \omega \leq 3$ the estimated jamming density of the considered polydisperse systems and their respective tridisperse counterparts are in perfect agreement and still within $1 \%$ for the widest studied size distributions of width $\omega=100$. We measured crystallinity and found that maximally equivalent tridisperse systems do not show signs of crystallization for $\omega \geq 1.4$ (data are shown in section 3.3), while equivalent bidisperse systems partially crystallize for $\omega \gtrsim 5$, since then, the small particles fit into interstices and thus do not hinder crystallization of the large particles [27]. We also confirmed

\footnotetext{
"Particles in a crystalline environment were identified using a method based on spherical harmonics given in [217]; the bond network was determined using a weighted Delaunay tessellation [129], effectively taking into account the strongly different radii of the particles.
} 

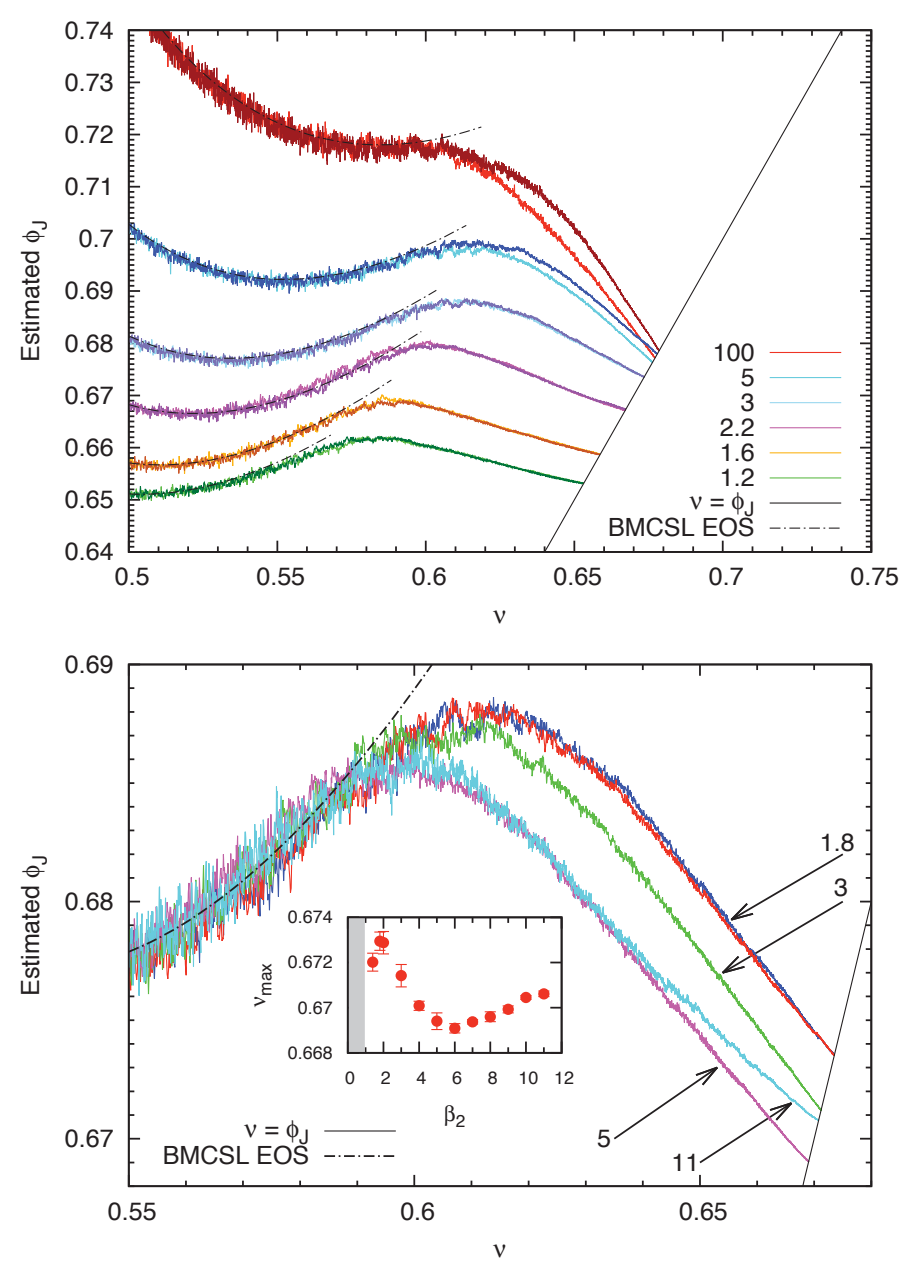

Figure 3.9: Estimated jamming density, $\phi_{J}^{\text {est }}$, plotted against volume fraction, $\nu$, (a, top) for systems with $N=4096$ and uniform size distribution (dark curves - dark-green, dark-orangered, dark-magenta, etc.) and their tridisperse maximal equivalents with the same $N$ (light curves - green, orangered, magenta, etc.), and (b, bottom) for the system with uniform size distribution with $\omega=3$ (dark blue) and some tridisperse equivalents with different $\beta_{2}$. Given in the legend of (a) are the size ratios $\omega$ corresponding to curves with increasing $\phi_{J}^{\text {est }}$. The values of $\beta_{2}$ in (b) are marked with arrows (for $\beta_{2}=1.8$ both tri- and polydisperse data are practically overlapping). In the inset of (b) the maximal reached volume fraction is plotted against the kurtosis $\beta_{2}$, where the error bars indicate the standard deviation of three different runs with random initial particle positions and velocities. The fluid theory (BMCSL EOS) is plotted as dot-dashed line.

that systems with uniform volume distribution and their tridisperse maximal equivalents have close equations of state in the range $1.2 \leq \omega \leq 10$ (data not 


\section{Equation of state and jamming density}

shown).

In Fig. 3.9(b) $\phi_{J}^{\text {est }}$ is plotted as a function of volume fraction for the uniformly polydisperse system with $\omega=3$ (top curve) and its tridisperse equivalents. We fix the scaled moments $k=2,3,5 \|$ and vary the $k=4$ moment, i.e., the kurtosis $\beta_{2}=\mu_{4} / \mu_{2}^{2}$, which describes both tailedness and peakedness of the size distribution function [218]. At high volume fractions, $\nu \geq \nu_{f}$, for glassy, metastable states, the higher-order moments, e.g, the kurtosis, play an important role. Even though the agreement is good for all equivalent systems with different $\beta_{2}$, it is close to perfect for the maximally equivalent system (with $\beta_{2}=1.8$ ), in both fluid and glassy regimes, including almost identical jamming densities ${ }^{* *}$. In the inset of Fig. 3.9(b), the maximal reached volume fraction is plotted against the kurtosis $\beta_{2}$ (in general, $\beta_{2} \geq 1$ ). The observed non-linear behavior can be explained by the vanishing presence of one species: for values of $\beta_{2}$ close to unity the system becomes nearly bidisperse due to vanishing concentration of the medium species, and for values of $\beta_{2}$ close to 12 the radius of the smallest species approaches zero (and becomes negative for $\beta_{2}>12$, so no physical solutions exist for this type of size distribution). Similar non-linear behavior of the jamming density was predicted and observed in Ref. [163].

A small mismatch in jamming densities (such with $Z>10^{13}$ ) can be seen in Figure 3.10(a), in the range $\omega \gtrsim 5$, where uniformly polydisperse systems jam at an about $0.5 \%$ higher density than their tridisperse maximal equivalents. We explain this deviation by linking the macro-scale jamming density with a micro-scale system property like "rattlers", i.e., particles that are free to float in the cage made by their jammed neighbors. Due to particle growth (or, equivalently, compression of the system), the velocity of all particles increases at each collision, for details see section 3.3. Therefore, in denser situations, the velocity of the rattlers, which collide much less frequent, vanishes compared to the velocity of the jammed particles, due to the thermostat that simply rescales all velocities by the same factor ${ }^{\dagger}$. The simplest way to identify rattlers is to look at their speed, which is close to zero, whereas the scaled speed of jammed particles is close to unity. The rattler criterion is chosen as $v_{r} \leq 10^{-4}$ and we confirmed that changing the above criterion in the range $10^{-3} \leq v_{r} \leq 10^{-6}$ does not affect the results.

Figure 3.10(b) shows the volume fraction of rattlers $\nu_{r}$ for many different

\footnotetext{
$\| \mu_{5} \equiv 0$ for the uniform size distribution.

**The importance of the fourth and fifth moments is also supported by estimates for the contact number density of static, soft granular packings, see Ref. [219] and references therein.

${ }^{\dagger}$ With this thermostat, the jammed network of non-rattlers equilibrates itself, while the rattlers are artificially "cooled" down. Alternative thermostats would "heat" rattlers without changing the system behavior since those would still collide much less than the jammed non-rattlers due to their larger mean free path.
} 

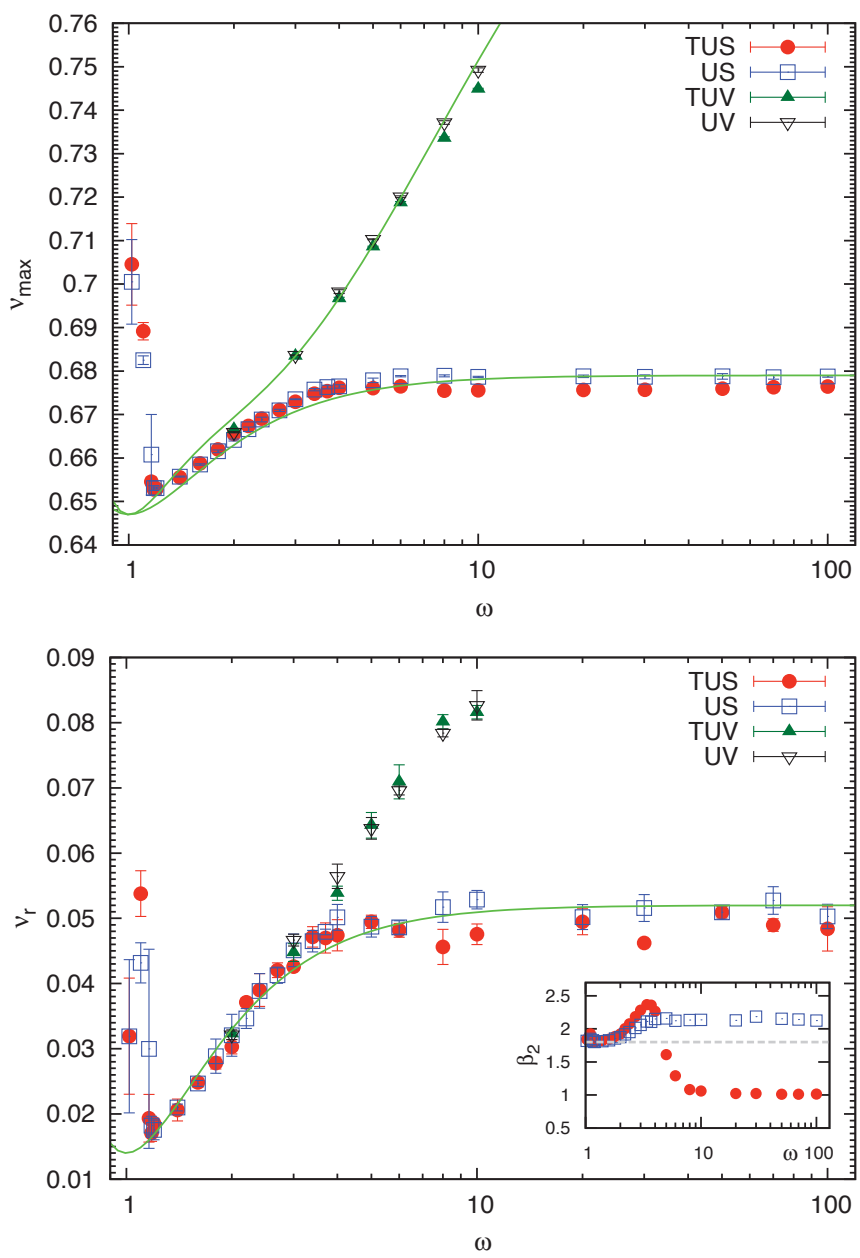

Figure 3.10: (a, top) Jamming density plotted against $\omega$ for systems the uniform size (US, $N=4096$ ) and uniform volume (UV, $N=8192$ ) distributions and for their tridisperse maximal equivalents (TUS and TUV, respectively). Solid lines are fits from Ref. [27] with parameters $\phi=0.647, \nu_{\mathrm{us}}^{\infty}=0.679$ and $\nu_{\mathrm{uv}}^{\infty}=0.86$. (b, bottom) Volume fraction $\nu_{r}$ of rattlers plotted against $\omega$ for systems considered in (a). The solid line is the same fitting function as in (a) for the US data, but with different parameters $\phi=0.014$ and $\nu_{\mathrm{r}}^{\infty}=0.052$. The error bars indicate the standard deviation of three different runs with random initial particle positions and velocities. The kurtosis of the US/TUS systems is plotted in the inset with rattlers $\left(\beta_{2}=1.8\right.$, dashed line) and with rattlers excluded (symbols).

size ratios, for the uniform size and uniform volume distributions and for their tridisperse maximal equivalents. Surprisingly, for $\omega \geq 1.2, \nu_{r}$ is very similar for poly- and the respective tridisperse systems. This shows that the maximal equivalent systems also have a very similar microstructure in 
that respect. (We also found that the poly- and the respective tridisperse structure factors have very similar low-wavenumber behavior, see section 3.3.) Moreover, $\nu_{r}$ correlates with the jamming density in Fig. 3.10(a), i.e., larger $\nu_{\max }$ correlates with larger $\nu_{r}$. Interestingly, $\nu_{r}$ for the US/TUS systems is described by the same functional form as the jamming density in Fig. 3.10(a). Note that when rattlers are excluded, the size distributions are not equivalent anymore, as quantified by the kurtosis in the inset of Fig. 3.10 (b). The kurtosis considering of non-rattlers only deviates from $\beta_{2}=1.8$ for both systems already for $\omega>2$. The tridisperse and polydisperse data develop differently from each other at $\omega \gtrsim 4$, and reach plateaus for $\omega \geq 10$. In the TUS system, number fractions $n_{i}$ change from their original values (with rattlers) 5:8:5 to 0:5:5, i.e., all small particles and about $40 \%$ of the medium particles become rattlers. The strong decrease of $\beta_{2}$ for the tridisperse systems (in contrast to a small increase for the polydisperse systems) explains the small but systematic differences between their $\nu_{\max }$-values for $\omega \gtrsim 4$. The non-rattlers form the jammed system [220] and their size distribution (and moments) are relevant for the jamming density. With other words, tridisperse systems are equivalent to polydisperse systems if the size distribution moments of the fraction of non-rattlers are matched. A-priori this is impossible, but observations that (i) the volume fractions of rattlers are almost identical and (ii) $\nu_{r}$ has the same functional form as the jamming density (for the US/TUS systems at least) provide a first step towards truly maximally equivalent tridisperse systems.

In conclusion, starting from different polydisperse systems, we defined equivalent and maximally equivalent hard sphere systems on the basis of identical first two and four scaled moments of their size distribution functions, respectively. By means of Event-Driven molecular dynamics simulations we confirmed that equivalent (bidisperse) systems match and predict the behavior of the polydisperse system in the fluid regime. Interestingly, maximally equivalent tridisperse systems match/predict polydisperse ones for much higher densities - in non-equilibrium, glassy states. Tridisperse systems do not suffer from partial crystallization, as observed for large size ratios in equivalent bidisperse systems [27], and even allow to predict the maximal jamming density within about $0.5 \%$ accuracy.

We identified the rattlers in glassy systems as the reason for this small but significant discrepancy. The size distribution of poly- and (maximally equivalent) tri-disperse systems with rattlers excluded are considerably different from the original one and from each other, as quantified by the kurtosis. The maximally equivalent tridisperse systems have almost the same volume fraction of rattlers as the polydisperse ones they mimick, which indicates a very strong similarity in the microstructures, with respect to caging, perco- 
lation, and volume fraction of non-rattlers. Thus, truly maximally equivalent systems are those with four identical scaled moments of the non-rattlers.

Important consequences of our research are: (i) a tridisperse theory is still analytically treatable (like for bidisperse systems, without further assumptions), whereas an arbitrary polydispersity would require arbitrarily many species and coupled equations; (ii) other transport coefficients (like heatconductivity, viscosity etc.) can be computed analytically in the framework of a tridisperse theory (which goes beyond the scope of this study) and it should be checked if the 4-moments equivalency postulate holds for them too; (iii) experimentally, three species are much easier to control and (according to our prediction) should resemble multi-disperse systems; (iv) the multiscale nature of wide polydispersities (continuous from very small to very large sizes) could be replaced by a discrete (three-scales only) picture (model system) that covers a narrower range of particle sizes - thus representing a working multi-scale theory with enormous reduction of complexity.

In future studies, the effect of the growth rate and especially the limit $\Gamma \rightarrow 0$ should be studied further and a comparison with experiments should be carried out with the goal to validate the present theoretical predictions and establish their relevance in the presence of more realistic contact interactions like friction. 


\subsection{Supplementary materials}

\subsubsection{Details on the event-driven simulations}

In our event-driven molecular dynamics simulations we used a modification of the Event-Driven $\mathrm{C}++$ code written by A. Donev et al. $[67,134]$, which is available for download at http://cherrypit.princeton.edu/Packing/ $\mathrm{C}++$ /.

In our work we consider, in the style of A. Donev [21], Molecular Dynamics in a simple bounded simulation domain embedded in a Euclidean space of dimensionality $d$, defined by the lattice vectors, $\lambda_{1}, \ldots, \lambda_{d}$. The simulation domain, or unit cell, is a collection of points with $d$ relative coordinates $\mathbf{r}$ in the interval $[0,1]$, and corresponding Cartesian coordinates

$$
\mathbf{r}^{(E)}=\sum_{k=1}^{d} r_{k} \boldsymbol{\lambda}_{k}=\Lambda \mathbf{r}
$$

where $\Lambda$ is a square invertible matrix representing the lattice, and contains the lattice vectors as columns. The volume of the unit cell is given by the positive determinant $V \equiv|\boldsymbol{\Lambda}|$. In particular, we consider a cubic unit cell in 3D $(d=3)$ with lattice vectors $\boldsymbol{\lambda}_{1}=(1,0,0), \boldsymbol{\lambda}_{2}=(0,1,0)$ and $\boldsymbol{\lambda}_{3}=(0,0,1)$.

Additionally, we use periodic boundary conditions (BCs) which are imposed to mimic an infinite system, i.e., a statistically homogeneous medium. One can interpret periodic systems as being "infinite" and covering all Euclidean space with identical copies of the unit cell and the particles in this unit cell, however the system size $\boldsymbol{\lambda}_{k}$ defines the minimum possible wavenumber (largest wavelength). Periodic BCs are handled by considering the unit cell of the packing and considering each contact between an original $i$ and an image particle $\tilde{j}$ to be a contact between particles $i$ and $j$. The vector of $d$ integers $\mathbf{n}_{i j}$ specifies how many unit cells the contact $\{i, j\}$ crosses over [21]. This way the relative position between particles $i$ and $j$ is

$$
\mathbf{r}_{i j}=\mathbf{r}_{i}-\mathbf{r}_{j}+\Lambda \mathbf{n}_{i j}
$$

with the positions $\mathbf{r}_{i}$ and $\mathbf{r}_{j}$ of the centers of particles $i$ and $j$, respectively.

Given velocities of the particles just before the contact, $\mathbf{v}_{1}$ and $\mathbf{v}_{2}$, and their masses $m_{1}$ and $m_{2}$, the velocities after the collision are derived [83] from conservation of linear momentum and definition of the restitution coefficient, $e_{n}$, yielding:

$$
\begin{aligned}
& \mathbf{v}_{1}^{\prime}=\mathbf{v}_{1}+\Delta \mathbf{p} / m_{1}, \\
& \mathbf{v}_{2}^{\prime}=\mathbf{v}_{2}-\Delta \mathbf{p} / m_{2},
\end{aligned}
$$

where $\Delta \mathbf{p}$ is the change of momentum. In the case of smooth (frictionless) 
particles only the normal component of the change of momentum $\Delta \mathbf{p}^{(n)}$ is affected during the collision (i.e., no angular velocity), and it is calculated as [83]:

$$
\Delta \mathbf{p}^{(n)}=-m_{12}\left(1+e_{n}\right) \mathbf{v}_{c}^{(n)},
$$

with the reduced mass $m_{12}=m_{1} m_{2} /\left(m_{1}+m_{2}\right)$, and the normal component of the relative velocity of the contact-point of the particles $\mathbf{v}_{c}^{(n)}$. The latter is calculated taking into account the expanding sphere surfaces (for growing particles) as:

$$
\mathbf{v}_{c}^{(n)}=\hat{\mathbf{n}}\left[\left(\mathbf{v}_{1}-\mathbf{v}_{2}\right) \cdot \hat{\mathbf{n}}-\left(v_{1}^{g r}+v_{2}^{g r}\right)\right],
$$

with the unit vector in the normal direction $\hat{\mathbf{n}}=\mathbf{r}_{i j} /\left\|\mathbf{r}_{i j}\right\|$, and the growing speed of radius $a_{i}$ of particle $i$

$$
v_{i}^{g r}:=\frac{d a_{i}}{d t}=\Gamma \frac{a_{i}}{a_{\max }} v_{0},
$$

where $a_{\max }$ is the largest particle radius at time $t$, and $v_{0}:=\sqrt{\frac{2 E}{3 M}}$ is the thermal velocity, defined via the total fluctuation kinetic energy, $E$, and the total system mass, $M$, and $\Gamma$ is the dimensionless growth (compression) rate.

The compressibility factor $Z \equiv p V / N k_{B} T$, with kinetic temperature $k_{B} T=2 E / 3 N$, is calculated from the total exchanged momentum in all interparticle collisions during a short time period $\Delta t$ :

$$
Z=1-\frac{\sum\left\|\Delta \mathbf{p}_{i j}\right\| l_{i j}}{2 E \Delta t}
$$

where the bar $l_{i j}=\left\|\mathbf{r}_{i j}\right\|$ accounts for the distance over which momentum (force) is transmitted. The time period $\Delta t$ is chosen so that the total change in the kinetic energy due to growth stays below $1 \%$.

\subsubsection{Size distribution parameters}

In order to facilitate the process of finding a maximally equivalent tridisperse system, we consider, in the style of Bartlett [49], the tridisperse distribution detailed in Table 3.2. This distribution has been chosen so that varying the number fractions $n_{1,2}$, and the non-dimensional radii weighed by number fractions, $\delta_{i}=n_{i} a_{i} /\langle a\rangle, i=1,2$, allows the mixture composition and volume fraction to change, while the total number density $\rho=N / V$ and mean radius $\langle a\rangle$ are fixed. Note that any tridisperse mixture can be expressed in this form, since this distribution has six degrees of freedom as desired (i.e., $2 s$ for $s=3$ species): $\rho,\langle a\rangle, \delta_{1,2}$ and $n_{1,2}$. This is equivalent to the set $\nu,\langle a\rangle,\left\langle a^{2}\right\rangle, \ldots$, 
$\left\langle a^{5}\right\rangle$, as used in Ref. [82], and these two sets are related as:

$$
\begin{gathered}
\left\langle a^{k}\right\rangle=\sum_{i=1}^{3} n_{i} a_{i}^{k}=\langle a\rangle^{k}\left(\frac{\delta_{1}^{k}}{n_{1}^{k-1}}+\frac{\delta_{2}^{k}}{n_{2}^{k-1}}+\frac{\left(1-\delta_{1}-\delta_{2}\right)^{k}}{\left(1-n_{1}-n_{2}\right)^{k-1}}\right), \quad k \geq 1 \\
\nu=\rho \frac{4 \pi}{3}\left\langle a^{3}\right\rangle .
\end{gathered}
$$

\begin{tabular}{lccc}
\hline & Species 1 & Species 2 & Species 3 \\
\hline Number density $\rho_{i}$ & $\rho n_{1}$ & $\rho n_{2}$ & $\rho\left(1-n_{1}-n_{2}\right)$ \\
Radius $a_{i}$ & $\langle a\rangle \frac{\delta_{1}}{n_{1}}$ & $\langle a\rangle \frac{\delta_{2}}{n_{2}}$ & $\langle a\rangle \frac{\left(1-\delta_{1}-\delta_{2}\right)}{\left(1-n_{1}-n_{2}\right)}$ \\
\hline
\end{tabular}

Table 3.2: Specification of a tridisperse mixture of hard spheres with fixed values for the total number density of particles, $\rho$, and mean radius $\langle a\rangle$. The properties of the mixture are then uniquely defined by the variables $\delta_{i}=n_{i} a_{i} /\langle a\rangle$ and $n_{i}=N_{i} / N$.

Consider polydisperse systems with a uniform size distribution of radii (e.g., same number of bigger spheres as smaller spheres in intervals $d a$ ), characterized by its extreme size ratio $\omega=a_{\max } / a_{\min }$, i.e., the ratio between the maximum and the minimum particle radius. The radius of the particles in such systems is distributed uniformly between $a_{\min }=\left(1-\omega_{0}\right)\langle a\rangle$ and $a_{\max }=\left(1+\omega_{0}\right)\langle a\rangle$, where $\omega_{0}=(\omega-1) /(\omega+1)$, and $2 \omega_{0}\langle a\rangle=a_{\max }-a_{\min }$ is the width of the (normalized) size distribution function $f(a)$ :

$$
f(a)=\frac{1}{2 \omega_{0}\langle a\rangle} \Theta\left(a_{\max }-a\right) \Theta\left(a-a_{\min }\right),
$$

with the Heaviside step function $\Theta(x)=1$ for $x \geq 0$ and $\Theta(x)=0$ elsewhere. For the uniform size distribution, due to its simplicity, maximally equivalent systems can be found analytically. We do it by solving a system of four equations that matches the first four central scaled moments with the tridisperse system from Table 3.2. Central scaled moments are very simple for the uniform size distribution:

$$
\begin{aligned}
& M_{2}^{c}=(1 / 3) \omega_{0}^{2}, \\
& M_{3}^{c}=0, \\
& M_{4}^{c}=(1 / 5) \omega_{0}^{4}, \\
& M_{5}^{c}=0,
\end{aligned}
$$


with $M_{1}^{c}=0$, due to the definition:

$$
M_{k}^{c}=\frac{\left\langle(a-\langle a\rangle)^{k}\right\rangle}{\langle a\rangle^{k}}
$$

Therefore, maximally equivalent tridisperse systems to the uniform size distribution (with parameter $\omega$ ) can be obtained analytically (e.g., using Mathematica software):

$$
\begin{gathered}
n_{1}=\frac{5}{18}, \quad n_{2}=\frac{8}{18} \\
\delta_{1}(\omega)=\frac{1}{18}\left(5+\sqrt{15} \omega_{0}\right), \quad \delta_{2}=\frac{8}{18} .
\end{gathered}
$$

Note that here and further we pick a unique solution (for a given $\omega$ ) with $a_{1} \geq a_{2} \geq a_{3}$ (sorted radii). Furthermore, equivalent systems (with matched $k=2,3,5$ scaled moments) to a system with uniform size distribution with $\omega=3$, considered in Ref. [82] in Figure 1(b), are detailed below for different kurtosis $\beta_{2}=M_{4}^{c} /\left(M_{2}^{c}\right)^{2}$ :

$$
\begin{gathered}
n_{1}=\frac{1}{2 \beta_{2}}, \quad n_{2}=\frac{\beta_{2}-1}{\beta_{2}}, \\
\delta_{1}=\frac{6+\sqrt{3 \beta_{2}}}{12 \beta_{2}}, \quad \delta_{2}=\frac{\beta_{2}-1}{\beta_{2}} .
\end{gathered}
$$

Consider systems with uniform volume distribution of radii in the sense that the total volume occupied with those particles with radii between $a_{1}$ and $a_{1}+d a$ is equal to the total volume occupied by particles with radii between $a_{2}$ and $a_{2}+d a$, etc. These systems can be also characterized by their extreme size ratio $\omega=a_{\max } / a_{\min }$. This is a truncated power law size distribution, which due to its sharp edges with well defined $\omega$, could be obtained by ideal sieving from wider, smooth continuously distributed realistic distributions. Power law distributions appear in a diverse range of natural and man-made phenomena [221]. The radius of the particles in such systems is distributed between $a_{\min }=\frac{\omega+1}{2 \omega}\langle a\rangle$ and $a_{\max }=\frac{\omega+1}{2}\langle a\rangle$ and the (normalized) size distribution function $f(a)$ is expressed as:

$$
f(a)=\frac{\langle a\rangle^{2}}{2 \omega_{0}} a^{-3} \Theta\left(a_{\max }-a\right) \Theta\left(a-a_{\min }\right) .
$$

Tridisperse maximally equivalent systems to polydisperse ones with uniform volume radii distribution, as considered in Ref. [82] in Figure 2, are detailed in Table 3.3. First four raw scaled moments $M_{k}=\left\langle a^{k}\right\rangle /\langle a\rangle^{k}$ for the uniform 
volume radii distribution are:

$$
\begin{aligned}
& M_{2}=\frac{\ln \omega}{2 \omega_{0}}, \\
& M_{3}=\frac{(1+\omega)^{2}}{4 \omega}, \\
& M_{4}=\frac{(1+\omega)^{4}}{16 \omega^{2}}, \\
& M_{5}=\frac{(1+\omega)^{4}\left(1+\omega+\omega^{2}\right)}{48 \omega^{3}} .
\end{aligned}
$$

To avoid possible confusion, we note that in the distribution detailed in Eq. (3.43), what is actually uniformly distributed is the inverse area $z=a^{-2}$ :

$$
f(z)|d z|=f(a)|d a|, \quad d z=-2 a^{-3} d a .
$$

Therefore, $f(z)=f(a) /\left(2 a^{-3}\right)=$ const for $a_{\max }^{-2} \leq z \leq a_{\min }^{-2}$. Accordingly, a proper nomenclature for this type of size distribution is uniform inverse area distribution (per inverse-area interval). However, we stick to the uniform volume size distribution in the sense defined above Eq. (3.43), in order to talk about size distributions only.

\begin{tabular}{ccccc}
\hline$\omega$ & $n_{1}$ & $n_{2}$ & $\delta_{1}$ & $\delta_{2}$ \\
\hline 2 & 0.1427 & 0.4054 & 0.1988 & 0.4337 \\
3 & 0.08923 & 0.3544 & 0.1593 & 0.4181 \\
4 & 0.06189 & 0.3118 & 0.1345 & 0.4034 \\
5 & 0.04582 & 0.2777 & 0.1173 & 0.3902 \\
6 & 0.03548 & 0.2502 & 0.1044 & 0.3785 \\
8 & 0.02331 & 0.2086 & 0.08630 & 0.3584 \\
10 & 0.01661 & 0.1789 & 0.07400 & 0.3419 \\
\hline
\end{tabular}

Table 3.3: Specification of tridisperse mixtures of hard spheres maximally equivalent to polydisperse systems with uniform volume distribution of radii for various extreme size ratios $\omega$.

Log-normal distributions appear in studies of emulsions, granular materials, such as sediments [222] and particle growth processes [223]. Therefore, we also study this size distribution of sphere radii, which has the form [222]:

$$
f(a)=\frac{1}{\sigma \sqrt{2 \pi} a} \exp \left\{-\frac{\left[\ln \left(a / a_{0}\right)\right]^{2}}{2 \sigma^{2}}\right\},
$$



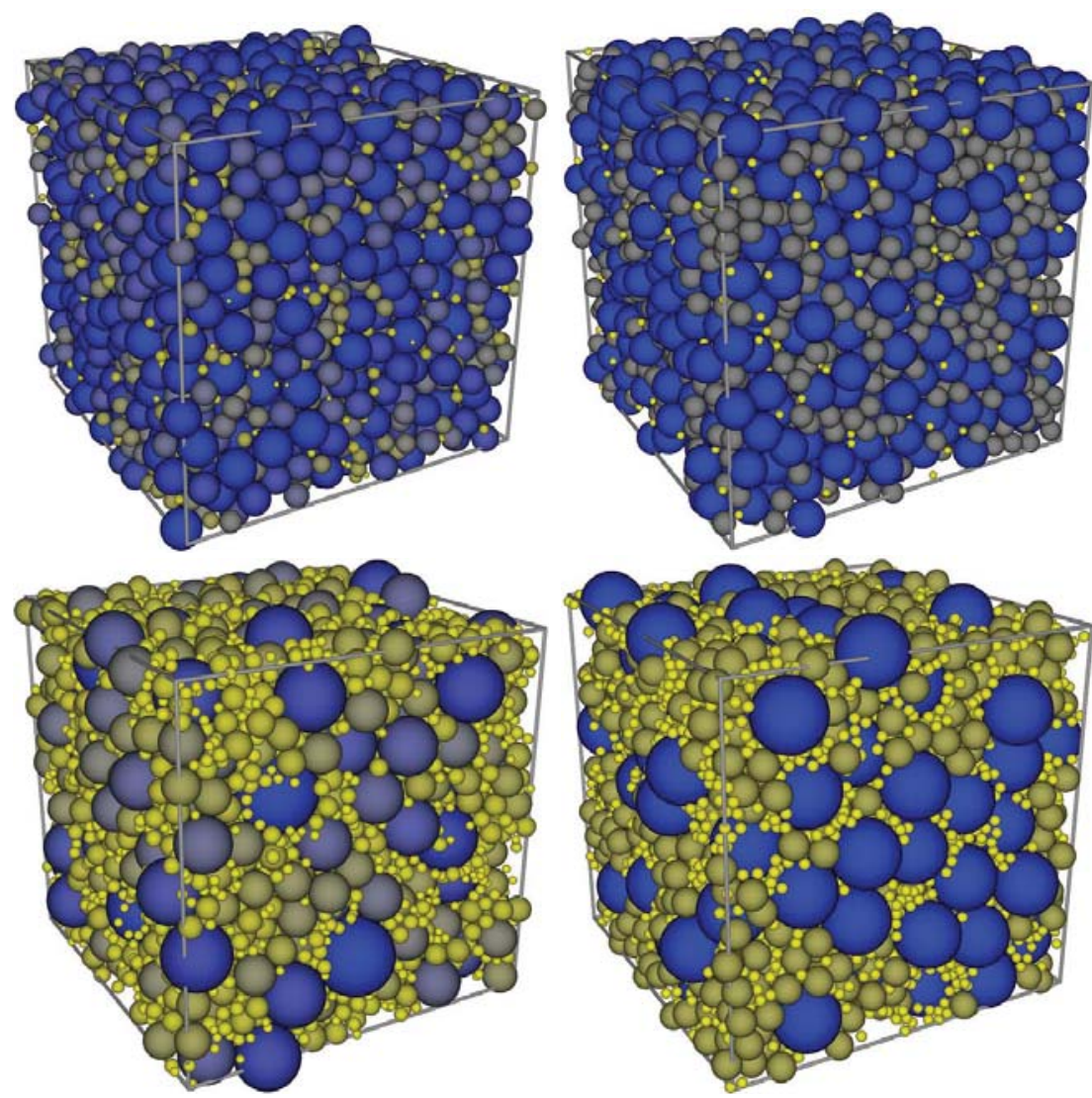

Figure 3.11: (top left) A polydisperse system with uniform size radii distribution with $\omega=10$ and $N=4096$ particles and (top right) its maximally equivalent tridisperse counterpart, and (bottom left) a polydisperse system with uniform volume radii distribution with $\omega=10$ and $N=8192$ particles and (bottom right) its maximally equivalent tridisperse counterpart. Color is by relative size, where darker grey particles are bigger - in color blue.

where $a_{0}$ is a reference radius setting the length scale:

$$
a_{0}=(1 / 2)\left(D_{32} D_{43}\right)^{1 / 2}\left(D_{32} / D_{43}\right)^{3} \text {, }
$$

and $\sigma$ is the dimensionless geometric standard deviation:

$$
\sigma=\sqrt{\ln \left(\frac{D_{43}}{D_{32}}\right)},
$$

with (often experimentally accessible) the volume-weighted mean diameter $D_{43}$ and the surface-weighted mean diameter $D_{32}$ defined in terms of the 


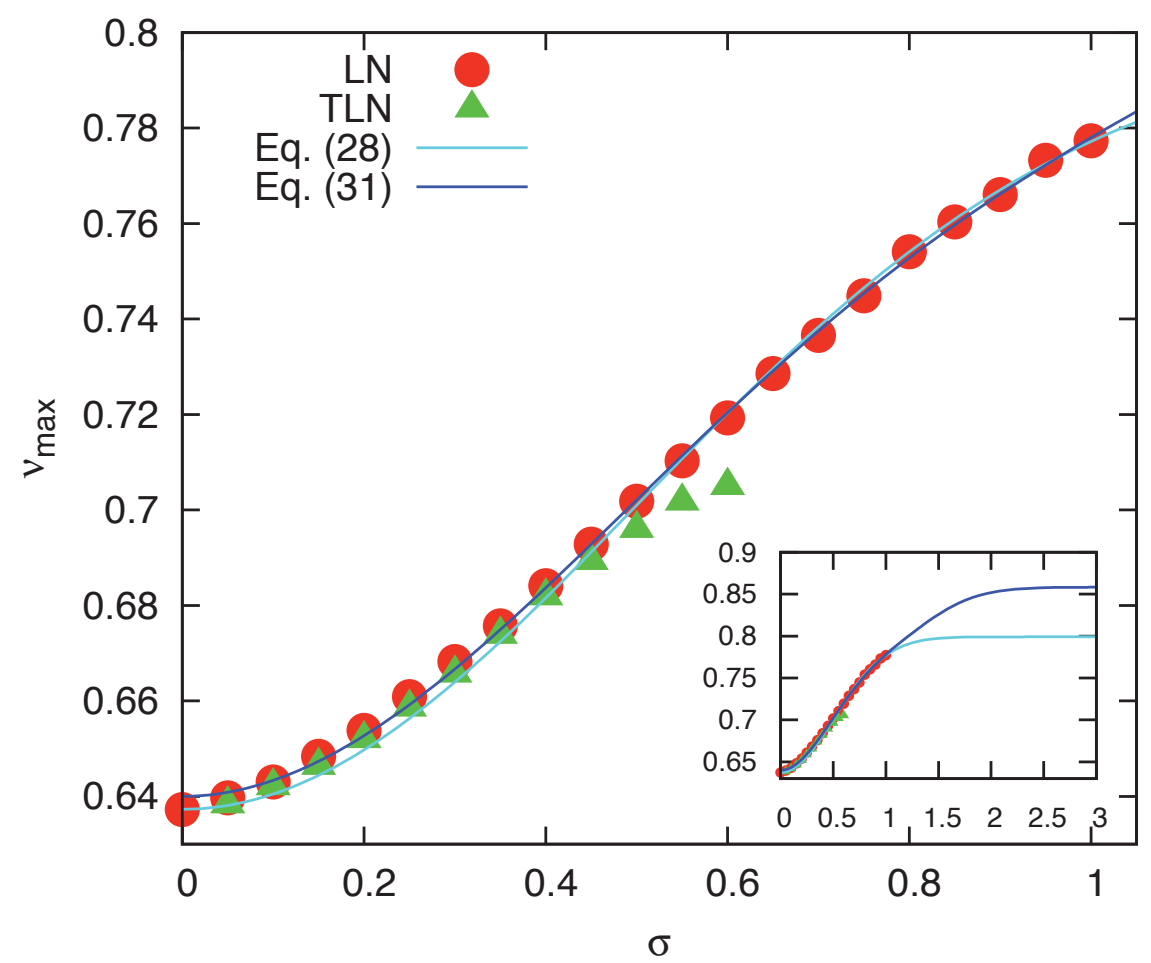

Figure 3.12: Jamming density plotted against $\sigma$ for systems with log-normal $(\mathrm{LN})$ radii distribution and for their tridisperse maximal equivalents (TLN), using $N=16384$. In the inset, the behavior of the fitting equations beyond the range of data is shown for larger values of $\sigma$, using the same axis and symbols. The deviation between LN and TLN data is due to the fact that the rattler-removed packings have highly different moments and thus being not true maximally equivalent anymore.

moments via:

$$
\begin{aligned}
& D_{43} \equiv 2\left\langle a^{4}\right\rangle /\left\langle a^{3}\right\rangle, \\
& D_{32} \equiv 2\left\langle a^{3}\right\rangle /\left\langle a^{2}\right\rangle .
\end{aligned}
$$

The log-normal moments $\left\langle a^{k}\right\rangle$ are given by

$$
\left\langle a^{k}\right\rangle=a_{0}^{k} \exp \left(k^{2} \sigma^{2} / 2\right) .
$$

Eq. (3.48) can often be used to estimate $\sigma$ for a real log-normal distribution of particle radii, using experimental sizing data, for example from lightscattering [222]. Note that while uniform size and uniform volume radii distributions are having sharp edges at $a_{\min }$ and $a_{\max }$, the log-normal distribution is smoothly increasing and decreasing. Tridisperse maximally equivalent systems to polydisperse ones with log-normal radii distribution, considered 
in this report in Fig. 3.12, are detailed in Table 3.4. Note that the number fraction $n_{1}$ of large species decreases very fast with increasing $\sigma$. This means that to have in the system, e.g., more than 10 large particles for $\sigma=1$, one has to use large total numbers of particles $N \geq 10^{6}$ (no data shown), which is computationally expensive.

\begin{tabular}{lcccc}
\hline$\sigma$ & $n_{1}$ & $n_{2}$ & $\delta_{1}$ & $\delta_{2}$ \\
\hline 0.05 & 0.1330 & 0.6620 & 0.1457 & 0.6653 \\
0.1 & 0.1040 & 0.6480 & 0.1263 & 0.6611 \\
0.15 & 0.07972 & 0.6255 & 0.1083 & 0.6543 \\
0.2 & 0.05980 & 0.5952 & 0.09197 & 0.6448 \\
0.25 & 0.04384 & 0.5585 & 0.07722 & 0.6329 \\
0.3 & 0.03138 & 0.5167 & 0.06405 & 0.6186 \\
0.35 & 0.02190 & 0.4713 & 0.05245 & 0.6021 \\
0.4 & 0.01488 & 0.4238 & 0.04236 & 0.5836 \\
0.45 & 0.009833 & 0.3758 & 0.03371 & 0.5634 \\
0.5 & 0.006306 & 0.3285 & 0.02639 & 0.5416 \\
0.55 & 0.003918 & 0.2832 & 0.02032 & 0.5186 \\
0.6 & 0.002355 & 0.2407 & 0.01535 & 0.4945 \\
0.65 & 0.001367 & 0.2017 & 0.01137 & 0.4695 \\
0.7 & 0.0007643 & 0.1666 & 0.008247 & 0.4440 \\
0.75 & 0.0004110 & 0.1357 & 0.005847 & 0.4180 \\
0.8 & 0.0002121 & 0.1090 & 0.004046 & 0.3920 \\
0.85 & 0.0001048 & 0.08627 & 0.002729 & 0.3659 \\
0.9 & 0.00004951 & 0.06731 & 0.001792 & 0.3401 \\
0.95 & 0.00002231 & 0.05177 & 0.001143 & 0.3147 \\
1.0 & 0.000009572 & 0.03923 & 0.0007078 & 0.2899 \\
\hline & & & &
\end{tabular}

Table 3.4: Specification of tridisperse mixtures of hard spheres maximally equivalent to polydisperse systems with log-normal distribution of radii for various $\sigma$.

Figure 3.12 shows the maximum density $\nu_{\max }$ as a function of $\sigma$ for systems with log-normal radii distribution and for their tridisperse maximal equivalents (with rattlers). A relatively fast compression rate, $\Gamma=16 \times 10^{-3}$ was used to achieve these configurations, i.e., a monodisperse system (with 
$\sigma=0)$ does not crystallize and reaches a random close packing. From our data, the jamming density $\nu_{\max }$ can be fitted by a function of $\sigma$ :

$$
\nu_{\max }(\sigma)=\nu_{\max }^{\infty}-\left(\nu_{\max }^{\infty}-\phi_{\mathrm{RCP}}\right) \exp \left(-2 \sigma^{2}\right),
$$

where the random close packing density $\phi_{\mathrm{RCP}}=\nu_{\max }(0)$ is 0.6373 (taken from data) and the maximum density, $\nu_{\max }^{\infty}=0.7990$, in the limit of $\sigma \rightarrow \infty$. Note that Eq. (3.52) has a similar form as previously reported equations for the jamming density for the uniform size and uniform volume radii distributions [27], i.e.,

$$
\nu_{\max }(x)=\nu_{\max }^{\infty}-\left(\nu_{\max }^{\infty}-\phi_{\mathrm{RCP}}\right) F(x) .
$$

Based on this observation, we speculate that this is a general form for the jamming density of some large class of polydisperse radii distributions, where a function $F(x)$ can be expressed in terms of moments of the size distribution. Particularly, for the log-normal radii distribution $F(x)$ can be expressed as (this is an arbitrary choice out of many):

$$
F_{\ln }(\sigma) \equiv \exp \left(-2 \sigma^{2}\right)=\left(\frac{D_{32}}{D_{43}}\right)^{2} .
$$

The deviation of Eq. (3.52) is within $\pm 0.7 \%$ for all data $0 \leq \sigma \leq 1$.

Nevertheless, we found that the jamming density for the log-normal radii distribution can also be fitted by another function, which is very close to Eq. (3.52) in the range $0 \leq \sigma \leq 1$, but differs for larger $\sigma$ :

$$
\nu_{\max }(\sigma)=\nu_{\max }^{\infty}+c_{1} \exp \left(-\sigma^{2}\right)+c_{2} \exp \left(-\sigma^{3}\right),
$$

with $\nu_{\max }^{\infty}=0.8583, c_{1}=-0.3516$ and $c_{2}=0.1332$. The deviation of Eq. (3.55) is within $\pm 0.4 \%$ for all data $0 \leq \sigma \leq 1$.

In Figure 3.11 we show final snapshots (near jamming) of polydisperse systems and their maximally equivalent tridisperse ones, which are considered in Ref. [82] in Figure 2.

\subsubsection{Measuring bond-orientational order}

In order to distinguish particles that are part of the crystal from those that belong to fluid or glass we utilize a method which is independent of the specific crystal structure and does not require the definition of the reference frame (i.e., rotationally invariant), provided by the following algorithm based on spherical harmonics $[217,224,225]$. The idea is to calculate for each 
particle $i$ a set of complex numbers

$$
\bar{q}_{l m}(i)=\frac{1}{N_{b}(i)} \sum_{j=1}^{N_{b}(i)} Y_{l m}\left(\hat{\mathbf{r}}_{i j}\right),
$$

where $Y_{l m}$ are spherical harmonics with components $m$ ranging from $-l \leq$ $m \leq l$, evaluated for the normalized direction vector $\hat{\mathbf{r}}_{i j}$ connecting the centers of mass of particles $i$ and $j$. The components of $\bar{q}_{l m}(i)$ depend on the relative orientation of particle $i$ with respect to its $N_{b}(i)$ neighboring particles [5]. For determining neighboring particles we utilize a weighted Delaunay tessellation [129] (otherwise called regular triangulation), effectively taking in account radii of particles. These triangulations provide information about inter-particle distances among a set of spheres. The CGAL external library was used for the construction of the triangulation. In order to account for periodic boundaries, we periodically repeat the simulation box in all three directions. We use $l=6$ because it allows to detect hep clusters and clusters with cubic symmetry (fcc, bcc, and sc) but also clusters with icosahedral symmetry, which can have nonzero spherical harmonics only for $l=6,10,12, \ldots[224]$. To this end, we construct a normalized complex vector $\mathbf{q}_{6}(i)$, with components $\tilde{q}_{6 m}(i)$ proportional to the $\bar{q}_{6 m}(i)$.

In a second step a dot product of the vectors $\mathbf{q}_{6}$ of neighboring particles $i$ and $j$ is computed:

$$
d_{6}(i, j)=\mathbf{q}_{6}(i) \cdot \mathbf{q}_{6}(j) \equiv \sum_{m=-6}^{6} \tilde{q}_{6 m}(i) \tilde{q}_{6 m}(j)^{*},
$$

where the ${ }^{*}$ indicates complex conjugation. Here, $d_{6}(i, j)$ is a normalized quantity correlating the local environments of neighboring particles [5]; it is a real number and is defined in the range $-1 \leq d_{6}(i, j) \leq 1$. By construction, $d_{6}(i, i)=1$. For example [5], in a perfect face-centered-cubic crystal, all the particles have the same environment and, therefore, the dot product between the vectors associated with any pair of particles is unity. The dot product decreases when thermal vibrations are present but, on average, it is close to unity if particles have a solid-like environment and around zero if particles have a liquid-like environment.

Now particles $i$ and $j$ are considered to be "connected" if $\mathbf{q}_{6}(i) \cdot \mathbf{q}_{6}(j)$ exceeds a certain threshold, in our case 0.65. A particle is labeled as solid-like if it has at least six connections. Finally, we define the degree of crystallinity $p_{c}$, or simply crystallinity, of a sample as the number of solid-like particles divided by the total number $N$.

Figure 3.13 shows the crystallinity $p_{c}$ as function of size ratio $\omega$ for systems with uniform size distribution characterized by its extreme size ratio $\omega$ and for 


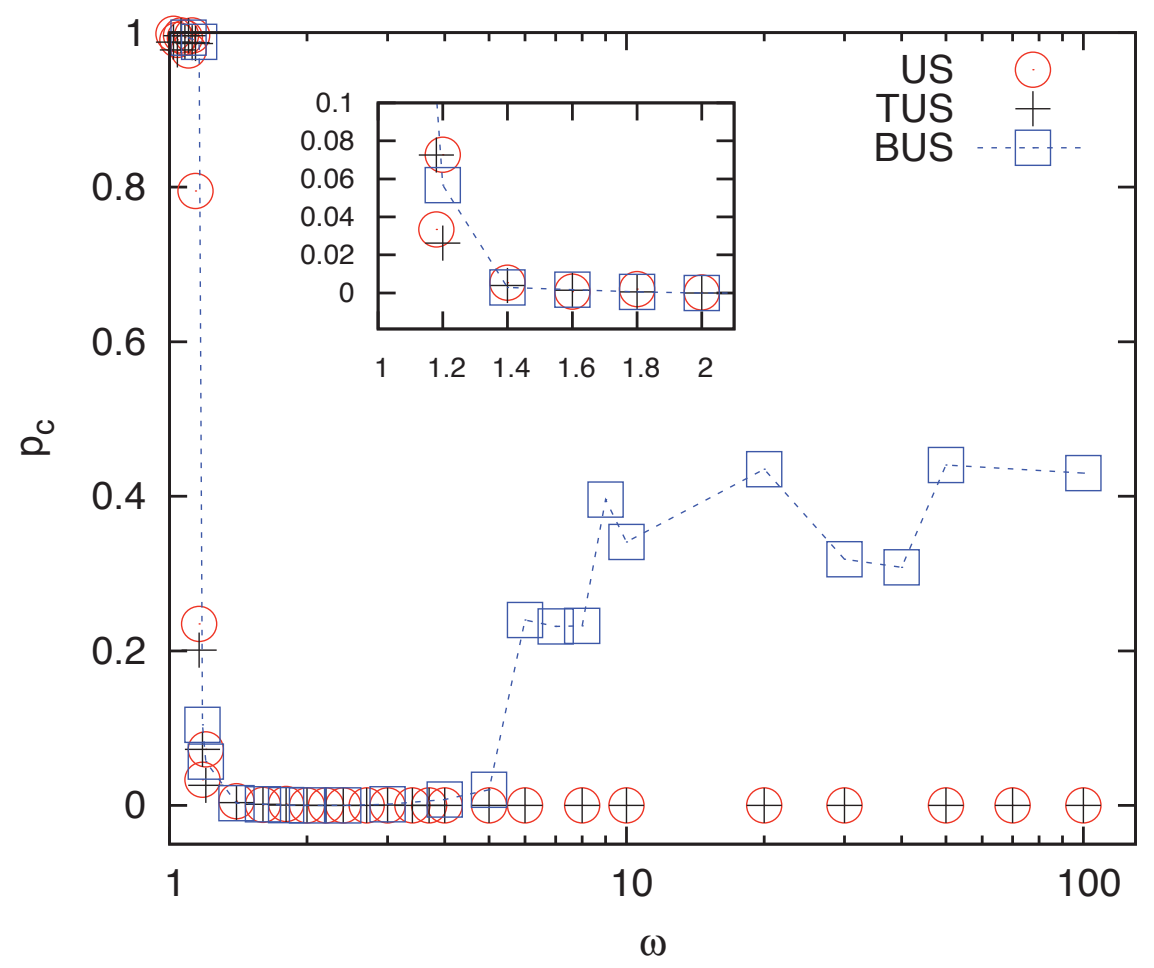

Figure 3.13: Crystallinity plotted against $\omega$ for systems with uniform size distribution (US), for their maximally equivalent tridisperse systems (TUS), and for equivalent bidisperse systems (BUS) previously considered [27]. In the inset we zoom into the low $\omega$ behavior using the same axis and symbols.

their maximally equivalent tridisperse systems (see section 3.3.2 for details) as well as for equivalent bidisperse systems considered in previous study [27]. Crystallinity data show that maximally equivalent tridisperse systems do not show any signs of crystallization for $\omega \geq 1.4$, while equivalent bidisperse systems partially crystallize for $\omega>5$.

\subsubsection{Structure factor and spectral density}

Recently S. Torquato and colleagues [226] have studied the small wavenumber $k$ behavior of the structure factor $S(k)$ of over-compressed amorphous hard sphere configurations for a wide range of densities up to the maximally random jammed state. They have found that a precursor to the glassy jammed state was evident long before the jamming density was reached as measured by a growing nonequilibrium length scale extracted from the volume integral of the direct correlation function. Their results are extended to different 
systems and to mixtures [227]. While the structure factor $S(k)$ measures local-number-density fluctuations, local-volume-fraction fluctuations provide the appropriate structural description of non-monodisperse packings because they account correctly for the size distribution of the particles. The analog of the structure factor in this context is the so-called spectral density, $\xi(k)$. We investigate if the polydisperse and the respective maximally equivalent tridisperse $S(k)$ and $\xi(k)$ are equivalent too.

Both structure factor and spectral density are numerically obtained using discrete Fourier transforms [228] according to

$$
\begin{gathered}
S(\mathbf{k})=\frac{\left|\sum_{j=1}^{N} \exp \left(-i \mathbf{k} \cdot \mathbf{r}_{j}\right)\right|^{2}}{N}(\mathbf{k} \neq 0), \text { and } \\
\xi(\mathbf{k})=\frac{\left|\sum_{j=1}^{N} \exp \left(-i \mathbf{k} \cdot \mathbf{r}_{j}\right) \hat{m}\left(\mathbf{k} ; R_{j}\right)\right|^{2}}{V}(\mathbf{k} \neq 0),
\end{gathered}
$$

where

$$
\hat{m}(\mathbf{k} ; R) \equiv \int_{\mathbb{R}^{d}} \exp (-i \mathbf{k} \cdot \mathbf{r}) \Theta(R-\|\mathbf{r}\|) d \mathbf{r},
$$

is the Fourier transform of the indicator function for a $d$-dimensional sphere of radius $R$. To calculate Eq. (3.60) we used results from Ref. [229]. Note that the shape of the domain, defined by a set of lattice vectors $\left\{\lambda_{i}\right\}$, restricts the wave vectors such that $\mathbf{k} \cdot \boldsymbol{\lambda}_{i}=2 \pi n$ for all $i$, where $n \in \mathbb{Z}$. To obtain spherically symmetric forms of the structure factor and spectral density, we angularly average over all wave vectors within a spherical shell of thickness $2 \pi /\left\|\boldsymbol{\lambda}_{i}\right\|$ in reciprocal space. The wave number $k$ is the magnitude of the wave vector $\mathbf{k}$, i.e., $k=\|\mathbf{k}\|$.

Figures $3.14,3.15,3.16,3.17$ show the structure factor $S(k)$ and the spectral density $\xi(k)$ for several polydisperse systems with uniform size and uniform volume radii-distributions, and for their maximally equivalent tridisperse systems (with rattlers). ( $D=2\langle a\rangle$ is an effective length scale, taken here to be the average diameter.) An exhaustive study of these quantities is well beyond the scope of the present study. Nevertheless, we observe that for low $\omega \leq 3$ the polydisperse and the respective maximally equivalent tridisperse $S(k)$ and $\xi(k)$ are in good agreement for all $k$-values. With increasing $\omega$ the agreement gets worse for high $k$-values, but still the low $k$ behavior is similar, especially in the case of the uniform size distribution. This observation confirms that maximally equivalent systems are indeed very similar in their microstructure. 

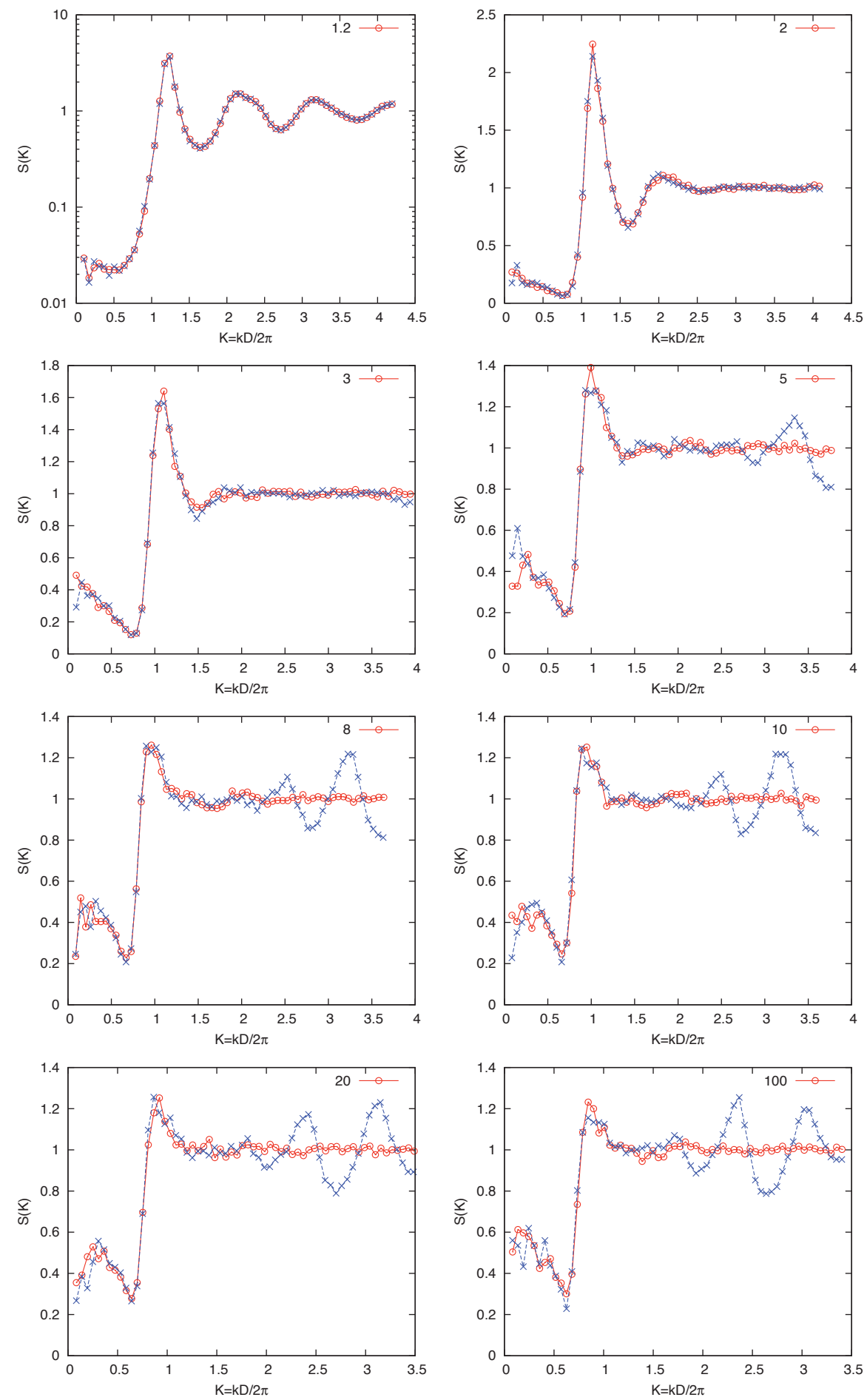

Figure 3.14: Structure factor as function of the scaled wavenumber for systems with uniform size distribution (circles) and for respective maximally equivalent tridisperse systems (crosses) for different $\omega$ given in the legends. 
Supplementary materials
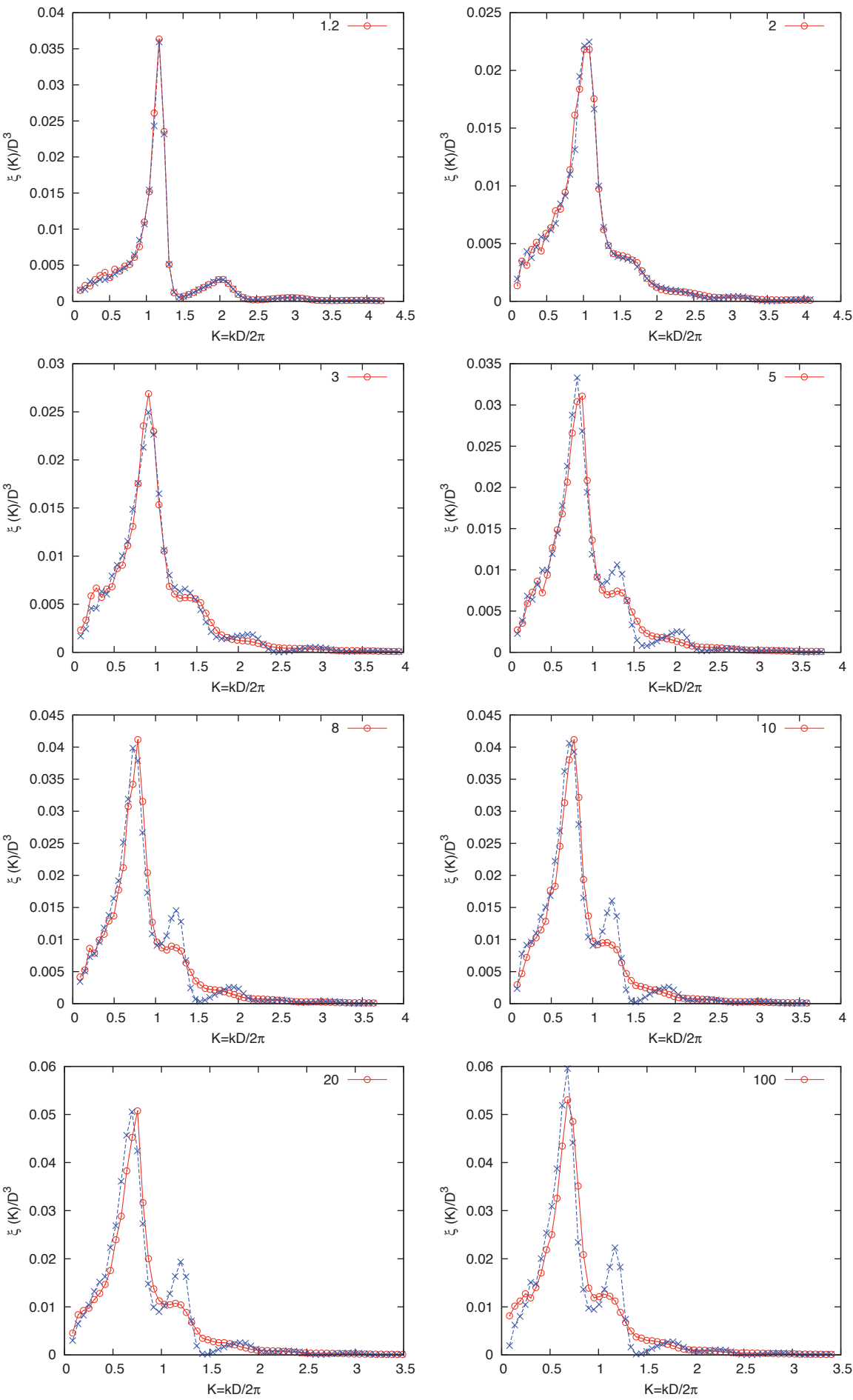

Figure 3.15: Spectral density as function of the scaled wavenumber for systems with uniform size distribution (circles) and for respective maximally equivalent tridisperse systems (crosses) for different $\omega$ given in the legends. 

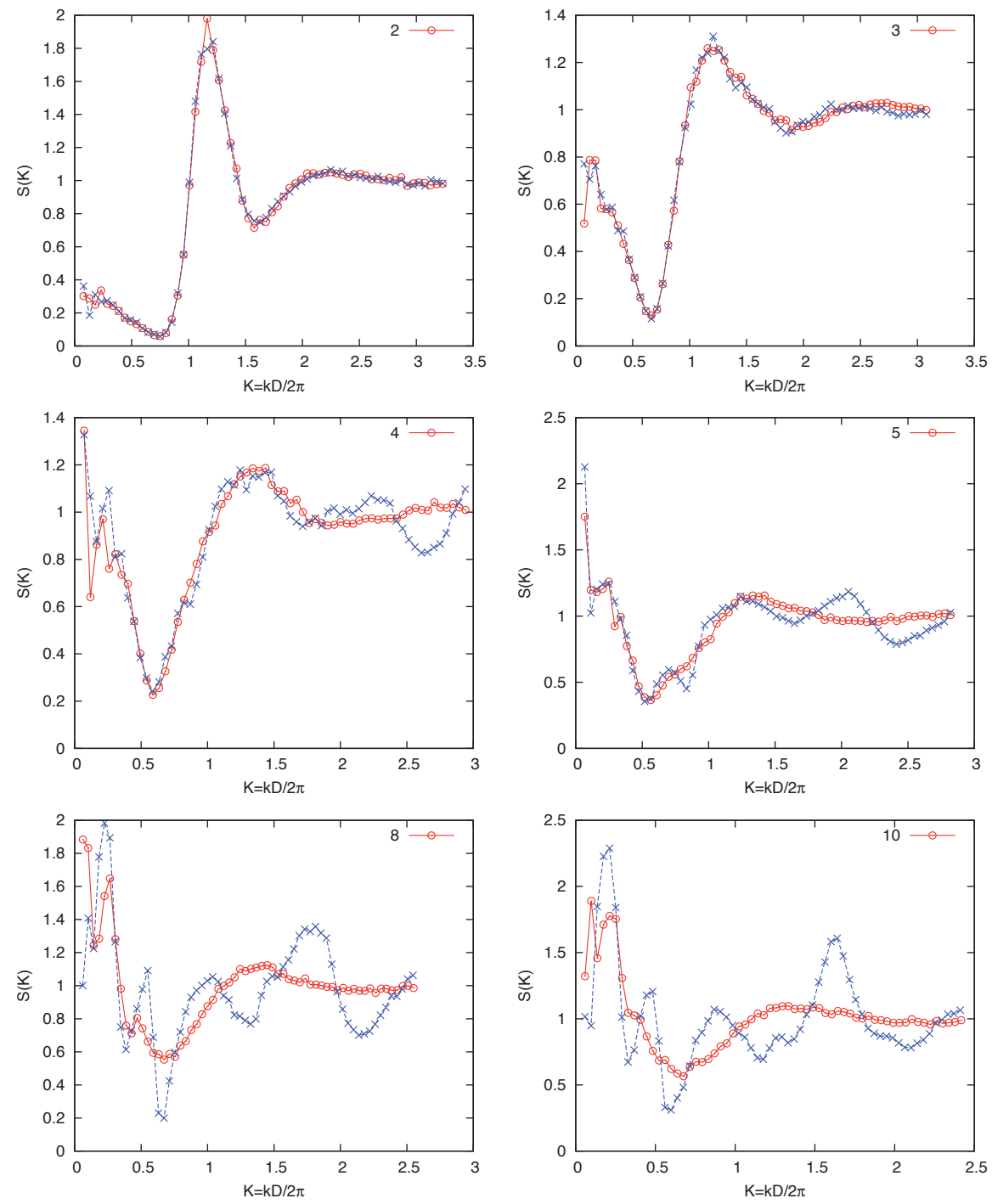

Figure 3.16: Structure factor as function of the scaled wavenumber for systems with uniform volume distribution (circles) and for respective maximally equivalent tridisperse systems (crosses) for different $\omega$ given in the legends. 

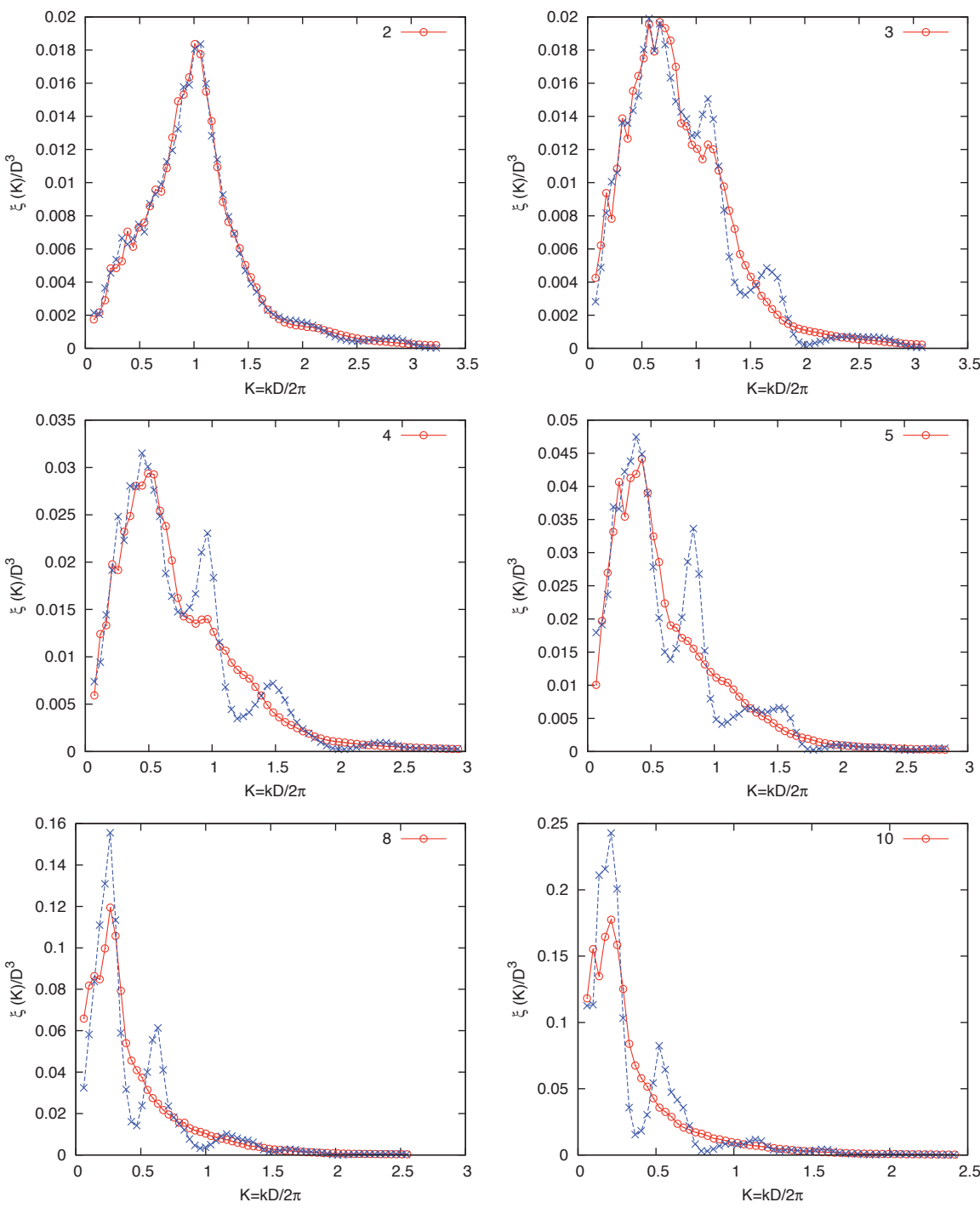

Figure 3.17: Spectral density as function of the scaled wavenumber for systems with uniform volume distribution (circles) and for respective maximally equivalent tridisperse systems (crosses) for different $\omega$ given in the legends. 



\section{Chapter 4}

\section{Structure characterization of hard sphere packings*}

The channel size distribution in hard sphere systems, based on the local neighbor correlation of four particle positions, is investigated for all volume fractions up to jamming. Every three particle combination of neighbors defines a channel, which can be relevant for the concept of caging. The analysis of the channel size distribution is very useful in distinguishing between gaseous, liquid, partially and fully crystallized, and glassy (random) jammed states. A common microstructural feature is observed in crystalline and glassy jammed states, suggesting the presence of "hidden" two-dimensional order in threedimensional random close packings.

\subsection{Introduction}

The hard-sphere particle interaction limit is a tremendously versatile physical model, being widely used for structural studies of liquids [2], glasses [10,230], colloids [231], granular materials [232], and many others [233, 234]. Its relevance in such a variety of physical systems suggests that many macroscopic properties arise by the fundamental fact of impenetrability of the systems constituents [3]. The ultimate goal then becomes to establish relations between physical properties and the geometry of the arrangement of hard bodies in two or three-dimensions. Many decades of research, heavily driven by numerical experiments [10], have lead to various geometrical structure variables, with different levels of success in either uniquely characterizing each state or in deriving macroscopic physical properties from them [4].

Our study is motivated by the structural phase transitions observed in molecular fluids and also replicated in hard-sphere systems under compres-

\footnotetext{
*Based on V. Ogarko, N. Rivas, and S. Luding, "Structure characterization of hard sphere packings in amorphous and crystalline states." Submitted, 2014.
} 
sion [120]. We also consider the analogous phenomena observed in granular materials, where the hard-sphere approximation is commonly used to successfully model complex rheological behaviours [232]. As the volume fraction is increased, hard-spheres enter an entropy minimization driven phase where glass formation competes with the nucleation and growth of the crystalline phase [235]. Hard-sphere models are known to successfully reproduce the main structural properties of these states for various physical systems, either for crystallization [11,12], or the amorphous solid phase transition [14]. One of the main reasons for using the hard-sphere model over classical condensed matter systems is that simulations - as also colloidal suspensions experiments, which are a very good approximations of the hard-sphere model $[5,12]$ - have particle-size spatial resolution, and thus the statics and dynamics can be studied from a microscopic perspective. Furthermore, due to the lack of long-range or non-binary interactions, and the simple geometry of the constituents, hard-sphere models are theoretically tractable [11,61, 236].

Inspired by the highly ordered and easily describable crystalline phase, many researchers have searched for inherent geometrical relations in disordered (amorphous) packings. The straightforward approach is to analyze the static structure factor, which is a direct measure of the local microstructure of particles [73]. The pair correlation function $g(r)$ is also a popular quantity for the analysis of non-crystalline materials [181,237]. The problem with such quantities is that the detailed three-dimensional information is lost as a result of statistical averages, as also by considering only pair of particles. In particular, they do not provide much information about the topology of the local structures in the particle-size scale, which are believed to distinguish different kinds of amorphous arrangements. It therefore becomes highly significant to exploit some other methods of three-dimensional characterization of these structures. Many attempts have already been made to quantify local or long-range ordering, providing further characterization of disordered packings [69, 70, 238-246].

In the following we analyze local arrangements of particles recognizing the importance of caging and voids in the overall structure and properties of the arrangement. Here we extend the previous studies [238, 239, 242] by considering all particle triples in the particles neighbourhood that do not include the central particle. The distribution of voids allows us to clearly distinguish between the different structural phases, as also between different kinds of crystals and the relative number of each specific ordering, in systems presenting partial or many types of crystallization. Our analysis shows that in amorphous states there is a preferred local structure of four co-planar particles. 


\subsection{Method of analysis}

We consider systems of $N$ non-overlapping spheres arranged in a threedimensional cubic space of volume $V$. The spheres are located at positions $\mathbf{x}_{i}$ and have radii $r_{i}$, with $r_{i} \equiv r$ for monodisperse systems, and for polydisperse systems radii probability density functions $s\left(r_{i}\right)$, which will be specified. Our main parameter is the sphere volume fraction, defined as $\nu=(4 / 3) \pi \sum_{1}^{N} r_{i}^{3} / V$.

To determine the neighbors of a particle, we first compute the weighted Delaunay triangulation of the set of points corresponding to the centers of the particles, $\left\{\mathbf{x}_{i}\right\}$ [129]. Neighboring particles are then defined as those particles connected by the edges of the triangulation. For each particle we consider every possible combination of three neighbors, that is, all possible triangles that can be formed by the centers of any three of its neighbors. We refer to these triangles as neighbor-triangles. Notice that the neighbortriangles do not contain the central particle. We then proceed to quantify the overall mobility of the particle by defining all channels through which the particle can go through. A channel is defined as the area in the plane of a neighbor-triangle by which the central particle could move. This is computed by considering the Apollonius circle, i.e, the circle which is simultaneously tangent to all other three circles defined by the projection of the three spheres in the neighbor-triangle plane. There are at most eight possible Apollonius circles for each case, which are obtained analytically by solving a system of three quadratic equations [247]. From the set of eight possible solutions we choose the one which correspond to the circle that does not contain any particle center of the neighbor-triangle, as it is the only one that corresponds to our definition of channel ${ }^{\dagger}$. The radius of the respective channel is then defined as the radius of this circle, $R_{j}$, as shown in Fig. 4.1 .

Having obtained $R_{j}$ for all neighbor-triangles of every particle, we then compute the normalized probability distribution function of (scaled) channel sizes, $f\left(R_{j} / r_{i}\right)$. The ratio $R_{j} / r_{i}$ is calculated for all neighbor triples $j$ with channel size $R_{j}$ of every particle $i$. Note that $R_{j} / r_{i}$ has a direct physical interpretation, as less than unity corresponds to a closed channel, while greater than unity corresponds to an open channel, through which eventually the central particle could escape. Furthermore, the function $f$ is well defined for spheres with any size distribution, since the radius of the central particle $i$ is scaled out. We analyze both the individual structure of $f$ as also its evolution with volume fraction, for various particle systems, in mono- and poly-disperse cases.

In order to refine our definition of channels, we consider $f\left(R_{j} / r_{i}\right)$ for

\footnotetext{
${ }^{\dagger}$ It may happen that no such circle exists, which was occasionally observed for polydisperse systems. In this case, we skip the corresponding neighbor-triangle from the statistics.
} 

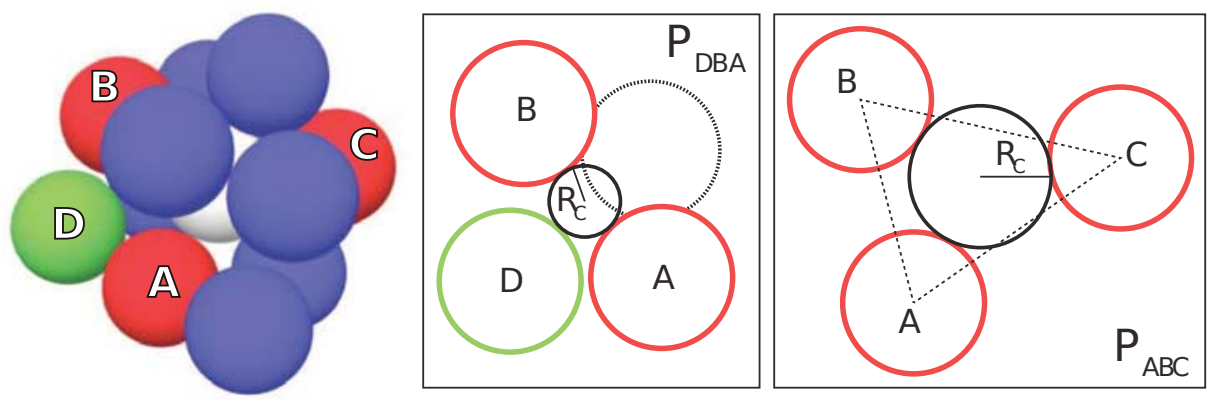

Figure 4.1: (left) The central particle (white) is shown together with its nearest neighbours, defined by Delaunay-edges. The channels for neighbourtriangles DBA (middle) and $\mathrm{ABC}$ (right) are shown in the neighbor-triangle plane. The particles $\mathrm{A}, \mathrm{B}$ and $\mathrm{C}$ are lying almost on the same plane with the central particle and are practically touching it, so the channel almost coincides with the central particle. This is not the case for the BDA triangle.

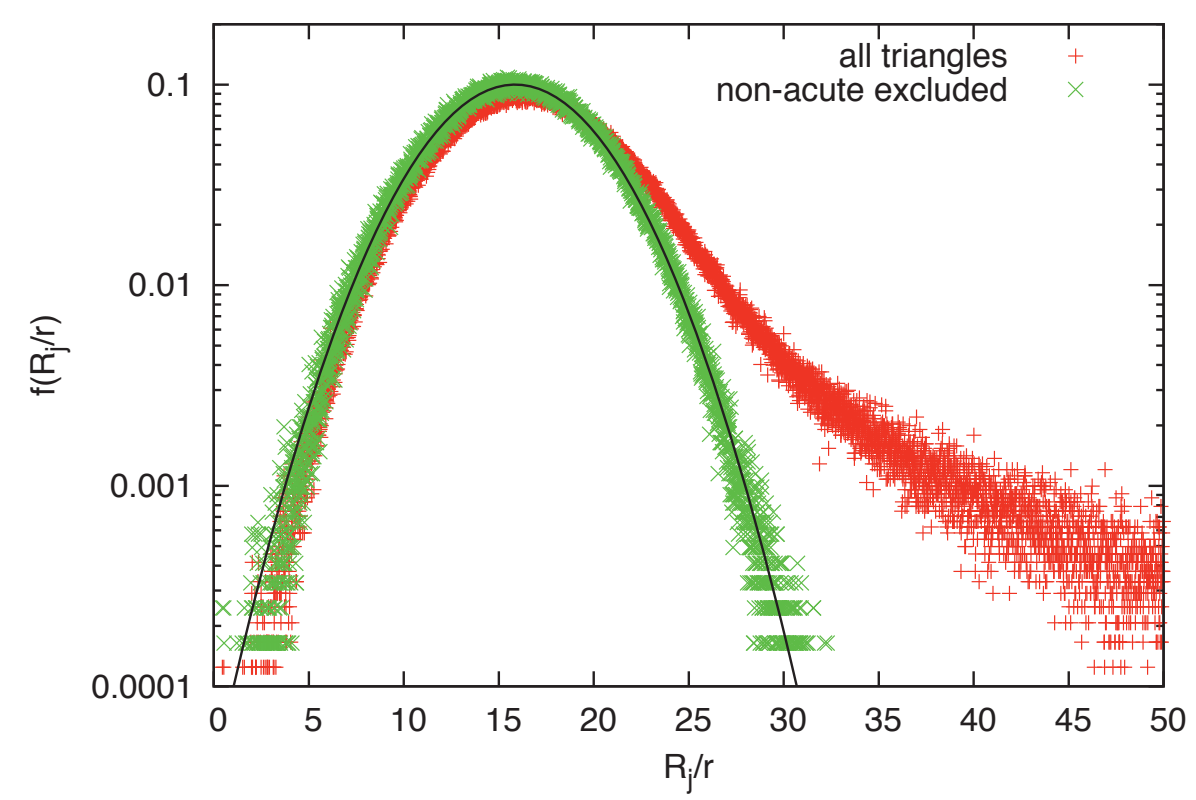

Figure 4.2: Normalized distribution of the channel sizes scaled with the particle radius in the gas regime $(\nu \approx 0.0014)$ using full statistics (red pluses) and with non-acute neighbor-triangles excluded (green crosses). The solid line is a Gaussian fit $g(x)=(\sigma \sqrt{2 \pi})^{-1} \exp \left[-(x-\mu)^{2} /\left(2 \sigma^{2}\right)\right]$ with parameters $\sigma \approx 4$ and $\mu \approx 15.9$. The bin-size used here is 0.01 . 
very low volume fractions, where no structure is expected (see Fig. 4.2). When considering all triangles the distribution presents a recognizable wide tail structure, but after excluding from the distribution the channels sizes that correspond to non-acute neighbor-triangles, i.e., those where one of the angles is greater than 90 degrees, the distribution becomes Gaussian, with high accuracy over three orders of magnitude $\ddagger$. The exclusion of non-acute triangles makes physical sense considering that channels defined by them cannot block the central particle, thus conflicting with our initial definition of a channel. For the rest of the analysis, non-acute triangles are never considered.

\subsection{Simulation details}

We use an event-driven molecular dynamics algorithm, as it is fundamentally suited for the simulation of hard sphere systems. The number of particles is by default $N=16^{3}=4096$, unless stated otherwise. Given the large amount of possible neighbor-triangles for each particle, the statistical significance rapidly increases with the number of particles in the system. We observed that 4096 particles was an adequate quantity, as increasing the number of particles did not produce any noticeable change in any of the results. Periodic boundary conditions are imposed to mimick an infinite system, i.e., a statistically homogeneous medium.

Starting from zero volume fraction, we compress the system towards a jammed state using a modification of the Lubachevsky-Stillinger algorithm $[81,134]$, which allows the radius of the particles to grow linearly in time with a dimensionless rate $\Gamma \S$ (while conserving the size distribution $s\left(r_{i}\right)$ ). The kinetic energy, $E$, is kept constant using a re-scaling thermostat procedure $[27,82]$.

If the growing is sufficiently slow, $\Gamma<0.0007$ [226], the monodisperse system stays in a gas-fluid state in approximate equilibrium during the densification phase, and exhibits a fluid-solid transition (crystallization) for volume fractions between $\nu_{\mathrm{f}} \approx 0.492$ (freezing point) and $\nu_{\mathrm{m}} \approx 0.543$ (crystal melting point), and finally reaches a stable solid (crystalline) phase $\nu_{\mathrm{m}}$ and the close-packing fraction $\nu_{\mathrm{cp}} \approx 0.7405$, corresponding to face-centered close packing. This corresponds to a thermodynamically stable branch in the hard sphere phase diagram [120]. On the other hand, for fast compression rates

\footnotetext{
${ }^{\mp}$ We use the following test to check if a triangle with edge lengths $a, b$ and $c$ is acute: $\left(a^{2}+b^{2}>\epsilon c^{2}\right) \&\left(c^{2}+b^{2}>\epsilon a^{2}\right) \&\left(a^{2}+c^{2}>\epsilon b^{2}\right)$, where $\epsilon=1-10^{-12}$ is used to account for numerical error.

$\S$ The growth rate is defined as $\Gamma=\frac{d a_{i}}{d t} \frac{a_{\max }}{a_{i}} \sqrt{\frac{3 M}{2 E}}$, with largest particle radius, $a_{\max }$, and total system mass, $M$.
} 
the system enters a metastable state for $\nu>\nu_{\mathrm{m}}$, which extrapolates continuously from the fluid branch and is conjectured to end at some random close packing state, around $\nu_{\mathrm{rcp}} \approx 0.64$, the interpretation of which is beyond the scope of this study, as its value depends on the details of the procedure [68].

\subsection{Results and discussion}

We now observe $f\left(R_{j} / r_{i}\right)$ for each of the previously described compression cases, first for monodisperse systems, and then extending our interpretations to polydisperse systems.

\subsubsection{Crystallization path}

As the volume fraction increases, the distribution of channel radii fundamentally changes, see Fig. 4.3 (a). The distribution changes to non-Gaussian for fluid densities above $\nu \approx 0.15-0.25$. We speculate that this change corresponds to the percolation gas-to-fluid transition observed by Woodcock [216] at similar packing fractions, although we did not investigate this in detail. As the volume fraction increases, two smooth humps continuously grow, that at higher $\nu>0.5$ evolve into two well defined peaks, centered above $R_{j} / r \approx 0.15$ and near $R_{j} / r=1$. These values can be understood in terms of the geometry of the local arrangements: $R_{j} / r \approx 0.15$ ideally corresponds to the size expected for three touching equal spheres, and thus the appearance and growth of this peak shows the appearance of triples in contact as well as the relative importance of density fluctuations. It is also the absolutely smallest possible channel size for equally sized spheres. The peak at unity, on the other hand, is obtained for three particles lying on the same plane with the central particle and practically touching it, i.e., when the channel essentially coincides with the central particle; we confirmed that the majority of particles corresponding to the peak at unity are indeed practically touching the central particle (data not shown).

As expected for very slow compression, $\Gamma=16 \times 10^{-6}$, the system exhibits (partial) crystallization near the melting point $\nu_{m} \approx 0.54$; crystallization at the freezing point is kinetically suppressed [215]. The distribution is able to capture the crystallization transition by the development of two split peaks. This was confirmed for many runs with different initial particle velocities and positions. The peak at $R_{j} / r \approx 0.15$, after crystallization, with increasing volume fraction, splits into two peaks at $R_{j} / r \approx 0.15$ and at $R_{j} / r \approx 0.41$, where the latter value corresponds to a square crystalline arrangement.

Finally, as the maximum volume fraction is reached (i.e, as the pressure diverges), the distribution is mostly dominated by steep peaks, see 

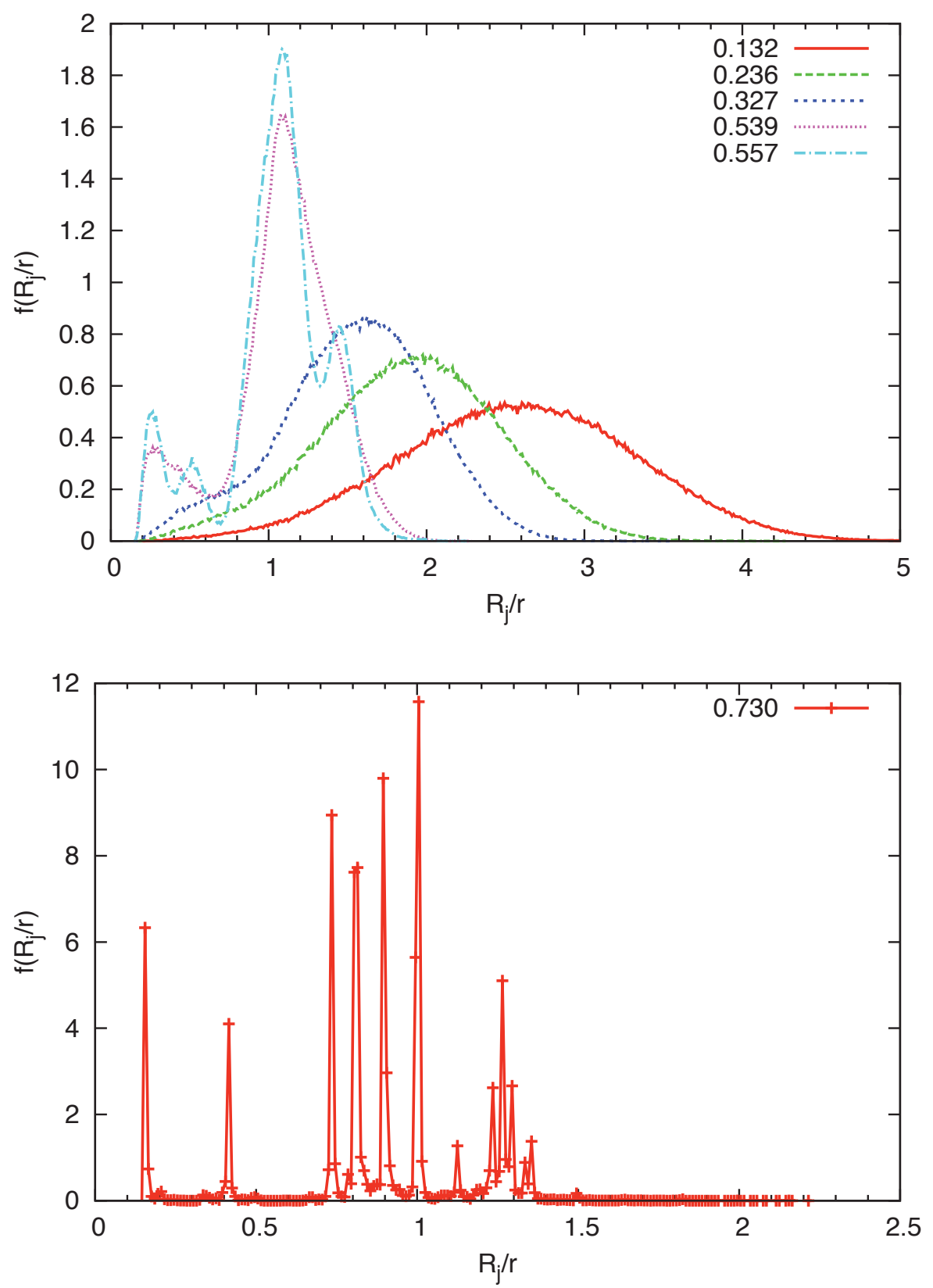

Figure 4.3: Normalized distribution of the channel sizes scaled with the particle radius for slow compression $\left(\Gamma=16 \times 10^{-6}\right)$ of a monodisperse system with various volume fractions given in the legend. The bin-size used here is 0.01 . 
Fig. 4.3 (b). In analogy with Bragg peaks from common diffraction techniques, these peaks can be traced to the crystal structures present in the particles' arrangement. We also confirmed that the obtained distribution allows to distinguish between FCC, HCP, BCC and SC crystal structures (data not shown).

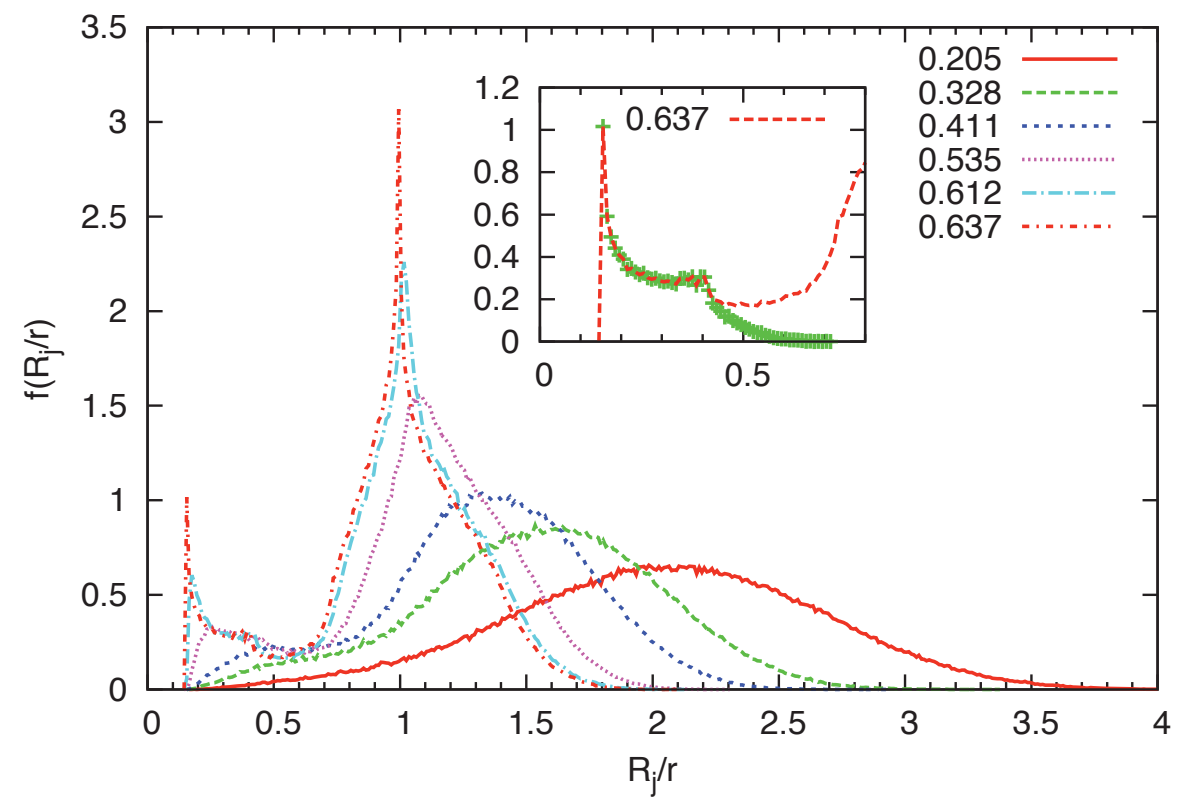

Figure 4.4: Normalized distribution of the channel sizes scaled with the particle radius for fast compression $\left(\Gamma=16 \times 10^{-3}\right)$ of a monodisperse system with various volume fractions $\nu$ given in the legend. The region of low $R_{j} / r$ is zoomed in the inset for the curve with $\nu=0.637$. The data with nonDelaunay neighbour-triangles excluded for the system with $\nu=0.637$ are shown in the inset with green pluses. The bin-size used here is 0.01 .

\subsubsection{Glassification path}

Let us now focus on the case of fast compression, $\Gamma=16 \times 10^{-3}$, for which crystallization is never reached. The channel size distributions for different volume fractions are shown in Figure 4.4. For volume fractions below $\nu \approx 0.2$, there is no appreciable difference with the slow compression case described in the previous section. For values above $\nu \approx 0.2$, the same peaks at $R_{j} / r \approx$ 0.15 and unity can be observed, but no other peaks are developed. The lack of defined peaks in the distribution, as in the previous slow compression cases, is a clear signal that the system remains, to very high degree, amorphous. Nevertheless, there exists partial order, as suggested by the high values of 


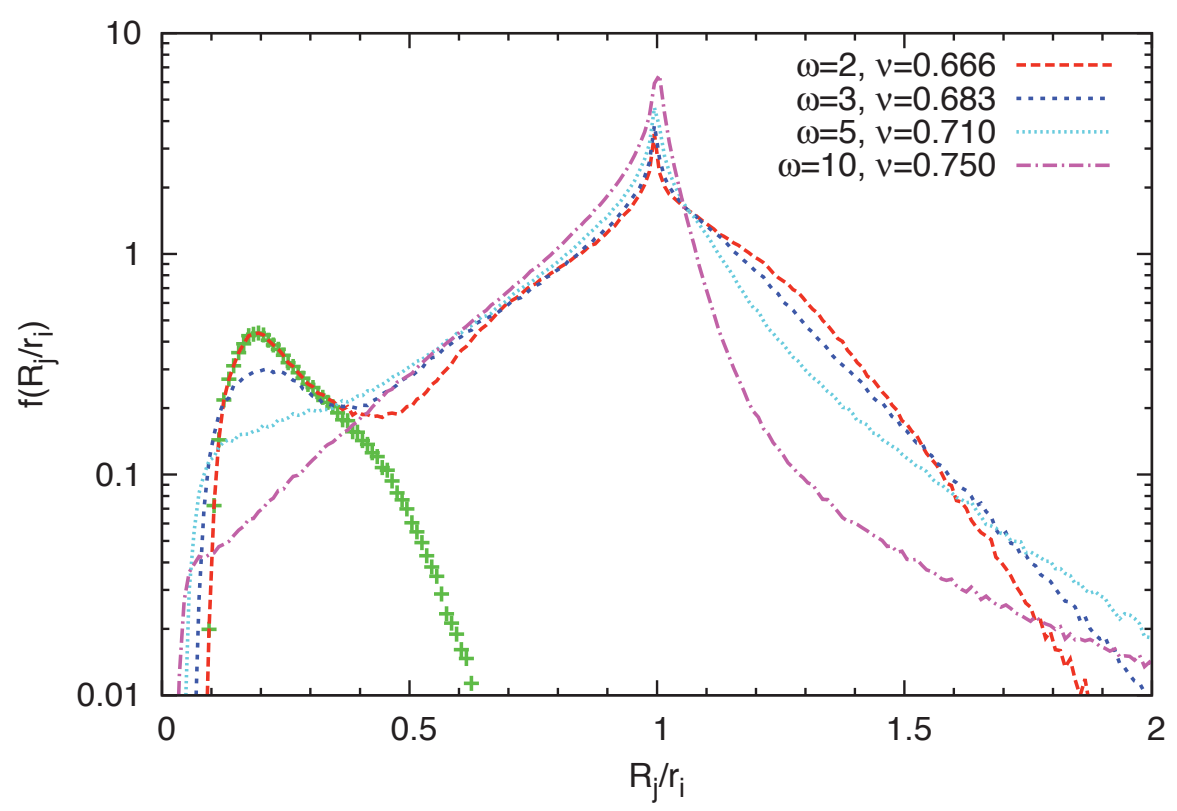

Figure 4.5: Normalized distribution of the channel sizes scaled with the central particle radius for random close packings of polydisperse systems from Ref. [82]. The data with non-Delaunay neighbour-triangles excluded for the system with $\omega=2$ are shown with green pluses. The width $\omega$ and the jamming packing fraction $\nu$ are shown in the legend. The number of particles is 8192 and the compression rate used to achieve these systems is $\Gamma=16 \times 10^{-6}$. The bin-size used here is 0.01 .

the peak at unity. While the peak at 0.15 can be interpreted in the same way as in the slow compression rate case, the peak at unity, on the other hand, is not as easily interpretable. While present in both crystalline and glassy configurations, its relative importance and shape are considerably different. The unity value corresponds to those configurations where three neighboring particles all touch the central particle and lie on the same plane with it. There are of course other cases where the value of $R_{j} / r_{i}$ could be one. By looking at the distribution of distance of each neighbor-triangle particle to the center particle, we confirm that by far the most common case is when the three particles are indeed touching the central one. This suggests the existence of "hidden" local order in random close packings that cannot be easily measured by order parameters because such local planes, corresponding to $R_{j} / r \approx 1$, are not oriented with respect to each other as in a periodic crystal structure. We have no explanation for this preference of the system structure, suggesting a direction of future research.

Let us now take a look at the structure of $f$ for values close to the minimal 
channel size, shown in the inset of Figure 4.4. The high values between the two ideal cases, corresponding to the three touching spheres $\left(R_{j} / r \approx 0.15\right)$ and a square arrangement $\left(R_{j} / r \approx 0.41\right)$, signals a significant presence of "intermediate" configurations. The drop at $R_{j} / r \approx 0.41$ is due to exclusion of non-acute neighbor-triangles from the statistics. A similar distribution of channel sizes, up to $R_{j} / r \approx 0.41$, was obtained in the studies of interstitial holes in random close packings of spheres [238, 239, 242]. They concluded that for monodisperse packings the spread in the channel sizes between 0.15 and 0.41 cannot be reduced to lead to a single distribution of channel sizes allied with mechanical stability.

\subsubsection{Polydisperse systems}

We now consider systems with the size distribution function

$$
s(a)=\Theta\left(a-a_{0}\right) \Theta\left(a_{0} \omega-a\right)\left(a / a_{0}\right)^{-n}(n-1) / a_{0}\left(1-\omega^{-n+1}\right),
$$

so that the distribution decays in the interval $a_{0}<a<a_{0} \omega$ as an inverse power law of order $n$, where $\Theta$ is the Heaviside step function. We confirmed the existence of the peak at unity also for random close packings of polydisperse systems (using $n=3$ ), as shown in Fig. 4.5. The peak at unity, as in the monodisperse case, corresponds to the most probable configuration of three neighboring particles where they all touch the central particle and lie on the same plane with it. At low $R_{j} / r_{i}$-values, the decreasing hump with increasing $\omega$ corresponds to the lower value of the minimal possible channel size for higher degrees of polydispersity, as the radii of the small particles are relatively smaller. If the channel size is scaled with the average particle radius, instead of the central particle radius, then structures (peaks) disappear and no relevant information on the local particle order is observed.

Finally, we tried to exclude from the channel statistics those neighbourtriangles that are not present in the Delaunay triangulation, i.e., only Delaunay triangles are considered. The results are shown in Figures 4.4 and 4.5 with green pluses. While for $R_{j} / r_{i} \lesssim 0.4$ the data almost identical to the one considering all neighbour-triangles, for larger values the data drops quickly to zero, so that all relevant information on the structure is lost. This shows that for the analysis of hard sphere packing microstructure it is not sufficient to consider Delaunay triangles alone, and new methods, like the one we presented here, are required to understand local neighborhood multi-particle structures. 


\subsection{Conclusions}

The analysis of channel size distributions was shown to be able to distinguishing between gaseous, liquid, partially and fully crystallized, and glassy (random) jammed states. Unlike the usually computed pair-distribution functions or structure factors, the channel size distribution is highly sensitive to changes in volume fraction, and presents unique features for each phase. States of partial crystallization can be recognized and characterized by the development and position of specific peaks, which can be traced to specific crystalline configurations, and could be used to quantify the degree of crystallization of the system. On the other hand, we confirm that random glassy configurations of isotropically (rapidly) grown hard particle systems present a common structural feature, as shown by looking at the channel size distributions. This corresponds to many three neighboring particles lying in the same plane as a central particle, almost touching it, which could be considered a first microscopic trace of crystals in a plane. As different planes are not oriented with dominant relative angles as in a crystal, there is no appreciable global crystal ordering. 



\section{Chapter 5}

\section{Conclusions and Outlook}

In this dissertation I have studied systems of polydisperse smooth hard spheres. In particular, I studied how to find contacts between particles in highly polydisperse systems and developed a fast and efficient contact detection algorithm for computer modelling; developed the macroscopic constitutive laws that are based on microscopic features, such as the moments of the particle size distribution; and developed a new analysis technique to study the microstructure in mono- and polydisperse systems with different physical behaviour, i.e., gaseous, liquid, glassy and crystalline states.

General conclusions are:

1. The developed contact detection algorithm based on the hierarchical grid data structure is very useful in finding contacts in particle systems with arbitrary polydispersity; and its optimal performance even increases with increasing the degree of polydispersity.

2. The nearly optimal parameters for best performance for the hierarchicalgrid-based contact detection algorithm can be obtained by using a simple rule: keep the same number of particles per cell at every level of hierarchy.

3. In the fluid regime, the theoretical BMCSL equation of state is in very good agreement (within 1\%) with the simulation results for many different systems tested (i.e., mono-, bi-, tri-, and polydisperse systems with various size distribution functions).

4. "Mapping" arbitrary polydisperse systems of hard spheres onto "equivalent" binary mixtures with the same first three moments of the particle size distribution function is very accurate concerning the equation of state of both systems being identical in the fluid regime.

5. In dense, glassy, non-equilibrium states one needs to consider at least the first five moments of the particle size distribution function. This 


\section{Conclusions and Outlook}

allows "maximally equivalent" ternary mixtures, with identical five moments as a polydisperse one, to predict well the polydisperse systems' equation of state and, notably, also the jamming density.

6. The maximally equivalent tridisperse systems have almost the same volume fraction of rattlers as the polydisperse ones they mimic, which indicates a very strong similarity in their microstructures; the behaviour of such systems near jamming is controlled by the non-rattlers.

7. The maximally equivalent tridisperse systems have very similar structure factors and spectral density as the polydisperse ones they mimic: for all wave numbers in case of low and moderate polydispersity and for low wave-numbers for high polydispersity. Together with item 6 this confirms strong similarity in their microstructure.

8. Sufficiently close to the jamming density the equation of state is independent of the size distribution function and depends only on one free parameter, the jamming density itself. The free volume equation of state of Salsburg and Wood is in very good agreement with our simulation results for all considered systems in two and three dimensions.

9. We provide practically useful analytical (empirical fit) equations for the jamming density for three types of continuous size distribution functions. All equations have only two free parameters, i.e., the random close packing density of a monodisperse system and the jamming density at extreme (infinite) polydispersity.

10. All obtained equations for the jamming density have a similar form; we suggest that the jamming density for systems with arbitrary size distribution can be expressed using only two free parameters (see item 9) and a few moments of the size distribution function. This was recently supported by theory and simulations [248].

11. We present new analysis technique to characterize the multi-particle microstructure in mono- and polydisperse hard-sphere systems, based on the local channels between particles. This analysis allows to distinguish between gaseous, liquid, partially and fully crystallized, and glassy (random) jammed states; it is highly sensitive to changes in volume fraction.

12. Using the analysis of item 11 a common microstructural feature is observed in crystalline and glassy jammed states, suggesting the presence of "hidden" two-dimensional order in random close packings of monoand polydisperse three-dimensional spheres. 
13. (preliminary research) With a Lubachevsky-Stillinger type protocol complemented by a random driving force, we reach volume fractions up to random close packing for strongly inelastic particle systems. Up to moderate volume fractions the equation of state is in good agreement with the inelastic version of the theoretical BMSCL equation of state; the volume fraction above which data start to deviate from the theory decreases with decreasing the coefficient of restitution [249].

14. (preliminary research) The random close packing volume fraction weakly decreases with decreasing the coefficient of restitution that quantifies the degree of inelasticity. The trends are such that the glass phase becomes very small for strongly inelastic particles [249].

In the different subjects we studied there are a few lines of research that could be continued. In what follows we give an account of the ones we consider the most important.

1. To investigate if the efficiency of the hierarchal grid algorithm for contact detection can be improved by combining it together with sortingbased methods (i.e., sweep and prune). The latter have a built-in bounding box overlap check, which might increase the performance of the existing algorithm.

2. To investigate more cache-friendly data structures than hash-tables for storing the hierarchical grid data. Good cache-friendly data structures are important for high performance and parallelization.

3. To study the full phase-space diagram for the jamming density and the physical state (i.e., glass, crystal or a coexistence) of ternary mixtures. This should help in understanding the limits of the five-moment approximation of polydisperse-by-tridisperse systems.

4. To study the equation of state of polydisperse systems at volume fractions above the glass transition density, especially for extremely low compression rates, which (at least theoretically predicted) may lead to fractionation. This can help in better understanding and prediction of some of the behaviors found in glassy materials.

5. To study what continuous size distributions lead to higher jamming density, i.e., 0.8 and above. More efficient packings may lead in practical applications to higher mechanical stability or strength in some materials.

6. To study the general accurate equation for the jamming density of arbitrary polydisperse mixtures, that can be described in terms of moments 


\section{Conclusions and Outlook}

of the size distribution function. This should involve only moments and other physically easily accessible (or already known) parameters, like for example, the random close packing density of monodisperse system.

7. To study weather the physical behavior of soft overlapping spheres above the jamming can be predicted using hard non-overlapping spheres. This is important in bridging the gap between statistical kinetic theory for fluids with, e.g., mode-coupling theory for glasses, or solid state theory for solids.

8. To study the influence of normal and tangential restitution coefficients on the equation of state and the jamming density of polydisperse mixtures. This is important since many real particulate materials are dissipative.

9. To continue studying the microstructural features observed in our new analysis of the micro-channel size distribution. This should eventually lead to understanding the nature of glass transition and the structure of the random close packings. The latter is important for understanding the geometrical principles at work in disordered packings and why density is limited at about 0.64 (in the monodisperse case). Furthermore, the channel sizes are a relevant microscopic property that determines the permeability for fluid flow through packings.

10. Since many realistic particles have various different non-spherical shapes, the issues above can be studied for non-spherical particles. This is a big challenge at least due to the existence of multiple shapes, which are difficult to characterize by a single function, unlike the case of different sizes which are described by a single size distribution function. Combining different sizes and different shapes is one of the biggest challenges for future research.

The reader of this dissertation will be able to find many other open questions, since they are naturally coming in the process of understanding some, and asking new questions. 


\section{Bibliography}

[1] J. D. Bernal, "The Bakerian lecture, 1962. The structure of liquids," Proc. R. Soc. A, vol. 280, no. 1382, pp. 299-322, 1964.

[2] J.-P. Hansen and I. R. McDonald, Theory of simple liquids. London: Academic Press, 1986.

[3] P. M. Chaikin and T. C. Lubensky, Principles of Condensed Matter Physics. Cambridge University Press, 2000.

[4] S. Torquato, Random Heterogeneous Materials: Microstructure and Macroscopic Properties. New York: Springer-Verlag, 2002.

[5] P. N. Pusey, E. Zaccarelli, C. Valeriani, E. Sanz, W. C. K. Poon, and M. E. Cates, "Hard spheres: crystallization and glass formation," Philos. T. R. Soc. A, vol. 367, no. 1909, pp. 4993-5011, 2009.

[6] A. Mehta, ed., Granular matter: An interdisciplinary approach. New York: SpringerVerlag, 1994.

[7] H. Hinrichsen and D. E. Wolf, eds., The Physics of Granular Media. Berlin: WileyVCH, 2004.

[8] H. A. Makse, J. Brujic, and S. F. Edwards, "Statistical mechanics of jammed matter," in The Physics of Granular Media, pp. 45-85, Berlin: Wiley-VCH Verlag, 2004 .

[9] R. Zallen, The Physics of Amorphous Solids. New York: Wiley, 1983.

[10] B. J. Alder and T. E. Wainwright, "Phase Transition for a Hard Sphere System," J. Chem. Phys., vol. 27, no. 5, pp. 1208-1209, 1957.

[11] W. G. Hoover and F. H. Ree, "Melting transition and communal entropy for hard spheres," J. Chem. Phys., vol. 49, no. 8, pp. 3609-3617, 1968.

[12] S. Auer and D. Frenkel, "Numerical prediction of absolute crystallization rates in hard-sphere colloids," J. Chem. Phys., vol. 120, no. 6, pp. 3015-3029, 2004.

[13] A. S. Clarke and H. Jónsson, "Structural changes accompanying densification of random hard-sphere packings," Phys. Rev. E, vol. 47, no. 6, pp. 3975-3984, 1993.

[14] M. D. Rintoul and S. Torquato, "Hard-sphere statistics along the metastable amorphous branch," Phys. Rev. E, vol. 58, no. 1, pp. 532-537, 1998.

[15] L. F. Tóth, Regular figures. Pergamon Press, 1964.

[16] T. Aste and D. Weaire, The pursuit of perfect packing. Bristol: IOP Publishing, 2000. 
[17] J. H. Conway and N. J. A. Sloane, Sphere Packings, Lattices, and Groups. New York: Springer-Verlag, 3rd ed., 1999.

[18] C. Zong, Sphere Packings. New York: Springer-Verlag, 1999.

[19] W. van Megen and S. M. Underwood, "Glass transition in colloidal hard spheres: Measurement and mode-coupling-theory analysis of the coherent intermediate scattering function," Phys. Rev. E, vol. 49, no. 5, pp. 4206-4220, 1994.

[20] G. Bryant, S. R. Williams, L. Qian, I. K. Snook, E. Perez, and F. Pincet, "How hard is a colloidal hard-sphere interaction?," Phys. Rev. E, vol. 66, no. 6, p. 060501, 2002.

[21] A. Donev, Jammed Packings of Hard Particles. PhD thesis, Princeton University, Princeton, United States, 2006.

[22] J. A. Gualtieri, J. M. Kincaid, and G. Morrison, "Phase equilibria in polydisperse fluids," J. Chem. Phys., vol. 77, no. 1, pp. 521-536, 1982.

[23] R. Blaak and J. A. Cuesta, "Continuous phase transition in polydisperse hard-sphere mixture," J. Chem. Phys., vol. 115, no. 2, pp. 963-969, 2001.

[24] P. N. Pusey, "The effect of polydispersity on the crystallization of hard spherical colloids," J. Phys. France, vol. 48, no. 5, pp. 709-712, 1987.

[25] P. N. Pusey and W. van Megen, "Phase behaviour of concentrated suspensions of nearly hard colloidal spheres," Nature, vol. 320, pp. 340-342, 1986.

[26] P. N. Pusey, "Colloidal suspensions," in Liquids, Freezing and Glass Transition (J. P. Hansen, D. Levesque, and J. Zinn-Justin, eds.), pp. 763-942, Amsterdam: NorthHolland, 1991.

[27] V. Ogarko and S. Luding, "Equation of state and jamming density for equivalent biand polydisperse, smooth, hard sphere systems," J. Chem. Phys., vol. 136, no. 12, p. $124508,2012$.

[28] M. Fasolo and P. Sollich, "Fractionation effects in phase equilibria of polydisperse hard-sphere colloids," Phys. Rev. E, vol. 70, no. 4, p. 041410, 2004.

[29] R. P. Sear, "Phase separation and crystallisation of polydisperse hard spheres," EPL (Europhys. Lett.), vol. 44, no. 4, p. 531, 1998.

[30] P. Bartlett, "Fractionated crystallisation in a polydisperse mixture of hard spheres," J. Chem. Phys., vol. 109, pp. 10970-10975, 1998.

[31] J. A. Cuesta, "Demixing in a single-peak distributed polydisperse mixture of hard spheres," Europhys. Lett., vol. 46, no. 2, pp. 197-203, 1999.

[32] P. B. Warren, "Fluid-fluid phase separation in hard spheres with a bimodal size distribution," EPL (Europhys. Lett.), vol. 46, no. 3, p. 295, 1999.

[33] P. Bartlett and P. B. Warren, "Reentrant melting in polydispersed hard spheres," Phys. Rev. Lett., vol. 82, no. 9, pp. 1979-1982, 1999.

[34] M. A. Bates and D. Frenkel, "Influence of polydispersity on the phase behavior of colloidal liquid crystals: A Monte Carlo simulation study," J. Chem. Phys., vol. 109, no. 14, pp. 6193-6199, 1998.

[35] P. Bartlett, "Freezing in polydisperse colloidal suspensions," J. Phys.: Condens. Mat., vol. 12, no. 8A, p. A275, 2000. 
[36] S.-E. Phan, W. B. Russel, J. Zhu, and P. M. Chaikin, "Effects of polydispersity on hard sphere crystals," J. Chem. Phys., vol. 108, no. 23, pp. 9789-9795, 1998.

[37] C. Voivret, F. Radjaï, J.-Y. Delenne, and M. S. El Youssoufi, "Multiscale force networks in highly polydisperse granular media," Phys. Rev. Lett., vol. 102, no. 17, p. 178001,2009 .

[38] Y. M. Zhang and T. J. Napier-Munn, "Effects of particle size distribution, surface area and chemical composition on Portland cement strength," Powder Technol., vol. 83, no. 3, pp. 245-252, 1995.

[39] C. Bentham, M. Dutt, B. Hancock, and J. Elliott, "Effects of size polydispersity on pharmaceutical particle packings," in Powders and Grains 2005 (R. Garcia-Rojo, H. J. Herrmann, and S. McNamara, eds.), Rotterdam: Balkema, 2005.

[40] F. Zhou, S. G. Advani, and E. D. Wetzel, "Slow drag in polydisperse granular mixtures under high pressure," Phys. Rev. E, vol. 71, no. 6, p. 061304, 2005.

[41] B. Remy, J. G. Khinast, and B. J. Glasser, "Polydisperse granular flows in a bladed mixer: Experiments and simulations of cohesionless spheres," Chem. Eng. Sci., vol. 66, no. 9, pp. 1811-1824, 2011.

[42] M. Bose, U. U. Kumar, P. R. Nott, and V. Kumaran, "Brazil nut effect and excluded volume attraction in vibrofluidized granular mixtures," Phys. Rev. E, vol. 72, no. 2, p. $021305,2005$.

[43] M. Y. Gundogdu, "Discharge characteristics of polydisperse powders through conical hoppers. Part 1: Predictions for fine, granular, free flowing powders," Particul. Sci. Technol., vol. 22, no. 4, pp. 339-353, 2004.

[44] W. M. Gelbart, A. Ben-Shaul, and D. Roux, eds., Micelles, Microemulsions and Monolayers. New York: Springer, 1994.

[45] N. Boden, R. J. Bushby, C. Hardy, and F. Sixl, "Phase behaviour and structure of a non-ionic discoidal amphiphile in water," Chem. Phys. Lett., vol. 123, no. 5, pp. 359-364, 1986.

[46] D. Vollmer, R. Strey, and J. Vollmer, "Oscillating phase separation in microemulsions. I. Experimental observation," J. Chem. Phys., vol. 107, no. 9, pp. 3619-3626, 1997.

[47] R. G. Petschek, P. Pfeuty, and J. C. Wheeler, "Equilibrium polymerization of chains and rings: A bicritical phenomenon," Phys. Rev. A, vol. 34, no. 3, pp. 2391-2421, 1986.

[48] S. Raschdorf and M. Kolonko, "A comparison of data structures for the simulation of polydisperse particle packings," Int. J. Numer. Meth. Eng., vol. 85, pp. 625-639, 2011.

[49] P. Bartlett, "A geometrically-based mean-field theory of polydisperse hard-sphere mixtures," J. Chem. Phys., vol. 107, no. 1, pp. 188-196, 1997.

[50] P. Sollich and M. E. Cates, "Projected free energies for polydisperse phase equilibria," Phys. Rev. Lett., vol. 80, no. 7, pp. 1365-1368, 1998.

[51] P. B. Warren, "Combinatorial entropy and the statistical mechanics of polydispersity," Phys. Rev. Lett., vol. 80, no. 7, pp. 1369-1372, 1998.

[52] P. Bartlett, "Thermodynamic properties of polydisperse hard spheres," Mol. Phys., vol. 97, no. 5, pp. 685-693, 1999. 
[53] P. Sollich, P. B. Warren, and M. E. Cates, "Moment free energies for polydisperse systems," Adv. Chem. Phys., vol. 116, pp. 265-336, 2001.

[54] N. Clarke, J. A. Cuesta, R. Sear, P. Sollich, and A. Speranza, "Phase equilibria in the polydisperse zwanzig model of hard rods," J. Chem. Phys., vol. 113, no. 14, pp. 5817-5829, 2000.

[55] J. L. Lebowitz, E. Helfand, and E. Praestgaard, "Scaled particle theory of fluid mixtures," J. Chem. Phys., vol. 43, no. 3, pp. 774-779, 1965.

[56] J. J. Salacuse and G. Stell, "Polydisperse systems: Statistical thermodynamics, with applications to several models including hard and permeable spheres," J. Chem. Phys., vol. 77, no. 7, pp. 3714-3725, 1982.

[57] T. Boublík, "Hard-sphere equation of state," J. Chem. Phys., vol. 53, no. 1, p. 471, 1970 .

[58] G. A. Mansoori, N. F. Carnahan, K. E. Starling, and T. W. Leland, "Equilibrium Thermodynamic Properties of the Mixture of Hard Spheres," J. Chem. Phys., vol. 54, no. 4, pp. 1523-1525, 1971.

[59] A. Santos, S. B. Yuste, and M. López de Haro, "Contact values of the particleparticle and wall-particle correlation functions in a hard-sphere polydisperse fluid," J. Chem. Phys., vol. 123, no. 23, p. 234512, 2005.

[60] A. Santos, "Note: An exact scaling relation for truncatable free energies of polydisperse hard-sphere mixtures," J. Chem. Phys., vol. 136, no. 13, p. 136102, 2012.

[61] M. López de Haro, S. B. Yuste, and A. Santos, "Alternative approaches to the equilibrium properties of hard-sphere liquids," in Theory and Simulation of HardSphere Fluids and Related Systems (A. Mulero, ed.), vol. 753 of Lecture Notes in Physics, pp. 183-245, Springer Berlin Heidelberg, 2008.

[62] G. D. Scott and D. M. Kilgour, "The density of random close packing of spheres," J. Phys. D: Appl. Phys., vol. 2, no. 6, p. 863, 1969.

[63] T. Aste, "Variations around disordered close packing," J. Phys.: Condens. Mat., vol. 17 , no. 24, p. S2361, 2005.

[64] L. V. Woodcock, "Glass transition in the hard-sphere model," J. Chem. Soc., Faraday Trans. 2, vol. 72, pp. 1667-1672, 1976.

[65] W. S. Jodrey and E. M. Tory, "Computer simulation of close random packing of equal spheres," Phys. Rev. A, vol. 32, no. 4, pp. 2347-2351, 1985.

[66] S. Torquato, T. M. Truskett, and P. G. Debenedetti, "Is random close packing of spheres well defined?," Phys. Rev. Lett., vol. 84, no. 10, pp. 2064-2067, 2000.

[67] M. Skoge, A. Donev, F. H. Stillinger, and S. Torquato, "Packing hyperspheres in high-dimensional euclidean spaces," Phys. Rev. E, vol. 74, no. 4, p. 041127, 2006.

[68] N. Kumar, O. I. Imole, V. Magnanimo, and S. Luding, "Effects of polydispersity on the micromacro behavior of granular assemblies under different deformation paths," Particuology, vol. 12, pp. 64-79, 2014.

[69] J. L. Finney, "Random Packings and the Structure of Simple Liquids. I. The Geometry of Random Close Packing," Proc. R. Soc. A, vol. 319, no. 1539, pp. 479-493, 1970 . 
[70] A. V. Anikeenko and N. N. Medvedev, "Polytetrahedral nature of the dense disordered packings of hard spheres," Phys. Rev. Lett., vol. 98, no. 23, p. 235504, 2007.

[71] Emory University, "Is Glass A Solid Or An Extremely Slow Moving Liquid?," ScienceDaily, 2007.

[72] C. R. Nugent, K. V. Edmond, H. N. Patel, and E. R. Weeks, "Colloidal glass transition observed in confinement," Phys. Rev. Lett., vol. 99, no. 2, p. 025702, 2007.

[73] N. E. Cusak, The Physics of Structurally Disordered Matter. Bristol: Hilger, 1987.

[74] R. Andersson, W. G. Bouwman, S. Luding, and I. M. de Schepper, "Stress, strain, and bulk microstructure in a cohesive powder," Phys. Rev. E, vol. 77, no. 5, p. 051303,2008 .

[75] R. Jullien, P. Jund, D. Caprion, and D. Quitmann, "Computer investigation of long-range correlations and local order in random packings of spheres," Phys. Rev. E, vol. 54, no. 6, pp. 6035-6041, 1996.

[76] S. Luding, K. Manetsberger, and J. Mllers, "A discrete model for long time sintering," J. Mech. Phys. Solids, vol. 53, no. 2, pp. 455-491, 2005.

[77] M. N. Bannerman, R. Sargant, and L. Lue, "DynamO: a free $\mathrm{O}(\mathrm{N})$ general eventdriven molecular dynamics simulator," J. Comput. Chem., vol. 32, no. 15, pp. 33293338,2011

[78] B. D. Lubachevsky, "How to simulate billiards and similar systems," J. Comput. Phys., vol. 94, no. 2, pp. 255-283, 1991.

[79] T. Pöschel and T. Schwager, Computational granular dynamics : models and algorithms. Springer-Verlag, 2005.

[80] S. Miller and S. Luding, "Event-driven molecular dynamics in parallel," J. Comput. Phys., vol. 193, no. 1, pp. 306-316, 2004.

[81] B. D. Lubachevsky and F. H. Stillinger, "Geometric properties of random disk packings," J. Stat. Phys., vol. 60, no. 5/6, pp. 561-583, 1990.

[82] V. Ogarko and S. Luding, "Prediction of polydisperse hard-sphere mixture behavior using tridisperse systems," Soft Matter, vol. 9, no. 40, pp. 9530-9534, 2013.

[83] S. Luding, "Collisions \& contacts between two particles," in Physics of Dry Granular Media (H. Herrmann, J.-P. Hovi, and S. Luding, eds.), vol. 350 of NATO ASI Series, pp. 285-304, Springer Netherlands, 1998.

[84] S. Luding, "Cohesive, frictional powders: contact models for tension," Granul. Matter, vol. 10, no. 4, pp. 235-246, 2008.

[85] P. A. Cundall and O. D. L. Strack, "A discrete numerical model for granular assemblies," Géotechnique, vol. 29, no. 1, pp. 47-65, 1979.

[86] D. Frenkel and B. Smit, Understanding Molecular Simulation. New York: Academic Press, 2002.

[87] V. Ogarko and S. Luding, "Data structures and algorithms for contact detection in numerical simulation of discrete particle systems," in Proceedings of the 6th World Congress on Particle Technology (WCPT6), (Nuremberg, Germany), 2010. 
[88] V. Ogarko and S. Luding, "A study on the influence of the particle packing fraction on the performance of a multilevel contact detection algorithm," in II International Conference on Particle-based Methods - Fundamentals and Applications (E. Oñate and D. R. J. Owen, eds.), (Barcelona, Spain), pp. 1-7, 2011.

[89] V. Ogarko and S. Luding, "A fast multilevel algorithm for contact detection of arbitrarily polydisperse objects," Comput. Phys. Commun., vol. 183, no. 4, pp. 931936, 2012.

[90] A. R. Thornton, T. Weinhart, V. Ogarko, and S. Luding, "Multi-scale methods for multi-component granular materials," Comput. Meth. in Mater. Sci., vol. 13, no. 2, pp. 197-212, 2013.

[91] D. Krijgsman, V. Ogarko, and S. Luding, "Optimal parameters for a hierarchical grid data structure for contact detection in arbitrarily polydisperse particle systems." Accepted in Comp. Part. Mech., 2014.

[92] A. R. Thornton, D. Krijgsman, A. te Voortwis, V. Ogarko, S. Luding, R. Fransen, S. González, O. Bokhove, O. Imole, and T. Weinhart, "A review of recent work on the discrete particle method at the university of twente: An introduction to the open-source package mercurydpm," in Proc. 6th Int. Conf. DEM (DEM6), (Golden, CO, USA), pp. 50-56, 2013.

[93] R. A. Gingold and J. J. Monaghan, "Smoothed particle hydrodynamics - Theory and application to non-spherical stars," Mon. Not. R. Astron. Soc., vol. 181, no. 2, pp. 375-389, 1977.

[94] J. R. Williams and R. O'Connor, "Discrete element simulation and the contact problem," Arch. Comput. Method Eng., vol. 6, no. 4, pp. 279-304, 1999.

[95] R. W. Hockney and J. W. Eastwood, Computer Simulation Using Particles. New York: McGraw-Hill, 1981.

[96] M. P. Allen and D. J. Tildesley, Computer Simulations of Liquids. Oxford: Clarendon Press, 1989.

[97] W. Form, N. Ito, and G. Kohring, "Vectorized and Parallelized Algorithms for MultiMillion Particle MD-Simulation," Int. J. Mod. Phys. C, vol. 4, no. 6, pp. 1085-1101, 1993.

[98] J. Stadler, R. Mikulla, and H. Trebin, "IMD: A software package for molecular dynamics studies on parallel computers," Int. J. Mod. Phys. C, vol. 8, no. 5, pp. 11311140, 1997.

[99] T. Iwai, C. W. Hong, and P. Greil, "Fast particle pair detection algorithms for particle simulations," Int. J. Mod. Phys. C, vol. 10, no. 5, pp. 823-837, 1999.

[100] B. Mirtich, "Efficient algorithms for two-phase collision detection," in Practical Motion Planning in Robotics: Current Approaches and Future Directions (K. Gupta and A. del Pobil, eds.), pp. 203-223, New York: Wiley, 1998.

[101] C. Ericson, Real-Time Collision Detection (The Morgan Kaufmann Series in Interactive 3-D Technology). San Francisco, CA, USA: Morgan Kaufmann Publishers Inc., 2004.

[102] H. Mio, A. Shimosaka, Y. Shirakawa, and J. Hidaka, "Optimum cell condition for contact detection having a large particle size ratio in the discrete element method," J. Chem. Eng. Jpn., vol. 39, no. 4, pp. 409-416, 2006. 
[103] M. Eitz and G. Lixu, "Hierarchical spatial hashing for real-time collision detection," in Proceedings of the International Conference on Shape Modeling and Applications 200\%, SMI 2007, (Lyon, France), pp. 61-70, 2007.

[104] K. He, S. Dong, and Z. Zhou, "Multigrid contact detection method," Phys. Rev. E, vol. 75 , no. 3, p. 036710, 2007.

[105] J. F. Peters, R. Kala, and R. S. Maier, "A hierarchical search algorithm for discrete element method of greatly differing particle sizes," Eng. Comput., vol. 26, no. 6, pp. 621-634, 2009.

[106] A. Munjiza, The Combined Finite-Discrete Element Method. John Wiley \& Sons Ltd., 2004.

[107] B. Muth, M.-K. Müller, P. Eberhard, and S. Luding, "Collision detection and administration methods for many particles with different sizes," in Proceedings of the 4th International Conference on Discrete Element Methods, DEM 2007, (Brisbane, Australia), 2007.

[108] T. Ulrich, "Loose octrees," in Game Programming Gems (M. Deloura, ed.), pp. 444453, Charles River Media, 2000.

[109] M. Moore and J. Wilhelms, "Collision detection and response for computer animation," Comp. Graph. (SIGGRAPH '88 Proceedings), vol. 22, no. 4, pp. 289-298, 1988.

[110] D. C. Rapaport, "Large-scale molecular dynamics simulation using vector and parallel computers," Comput. Phys. Rep., vol. 9, no. 1, pp. 1 - 53, 1988.

[111] S. Plimpton, "Fast parallel algorithms for short-range molecular dynamics," J. Comput. Phys., vol. 117, no. 1, pp. 1 - 19, 1995.

[112] Y. Tillé, Sampling Algorithms. Springer series in statistics, New York, NY: Springer Verlag, 2006.

[113] M. Kolonko, S. Raschdorf, and D. Waesch, "A hierarchical approach to simulate the packing density of particle mixtures on a computer," Granul. Matter, vol. 12, no. 6, pp. 629-643, 2010.

[114] J. M. N. T. Gray and A. R. Thornton, "A theory for particle size segregation in shallow granular free-surface flows," Proc. R. Soc. A, vol. 461, no. 2057, pp. 14471473,2005 .

[115] S. Miller and S. Luding, "Cluster growth in two- and three-dimensional granular gases," Phys. Rev. E, vol. 69, p. 031305, 2004.

[116] S. Luding, "Towards dense, realistic granular media in 2D," Nonlinearity, vol. 22, no. 12, pp. R101-R146, 2009.

[117] S. B. Savage and C. K. K. Lun, "Particle size segregation in inclined chute flow of dry cohesionless granular solids," J. Fluid Mech., vol. 189, pp. 311-335, 1988.

[118] J. T. Jenkins and D. K. Yoon, "Segregation in binary mixtures under gravity," Phys. Rev. Lett., vol. 88, no. 19, p. 194301, 2002.

[119] A. P. Gast and W. B. Russel, "Simple ordering in complex fluids - Colloidal particles suspended in solution provide intriguing models for studying phase transitions," Phys. Today, vol. 51, no. 12, pp. 24-30, 1998. 
[120] M. D. Rintoul and S. Torquato, "Metastability and crystallization in hard-sphere systems," Phys. Rev. Lett., vol. 77, no. 20, pp. 4198-4201, 1996.

[121] L. E. Silbert, D. Ertaş, G. S. Grest, T. C. Halsey, D. Levine, and S. J. Plimpton, "Granular flow down an inclined plane: Bagnold scaling and rheology," Phys. Rev. $E$, vol. 64, no. 5, p. 051302, 2001.

[122] B. Mirtich, "Efficient algorithms for two-phase collision detection," Tech. Rep. TR97-23, Mitsubishi Electric Research Laboratory, 1997.

[123] J. Williams and R. O'Connor, "Linear complexity intersection algorithm for discrete element simulation of arbitrary geometries," Eng. Comput., vol. 12, no. 2, pp. 185201, 1995.

[124] B. Mirtich, "V-clip: Fast and robust polyhedral collision detection," ACM T. Graphic., vol. 17, pp. 177-208, 1998.

[125] E. Gilbert, D. Johnson, and S. Keerthi, "A fast procedure for computing the distance between complex objects in three-dimensional space," IEEE J. Robot. Autom., vol. 4, no. 2, pp. 193-203, 1988.

[126] M. L. Gavrilova and J. G. Rokne, "Collision detection optimization in a multiparticle system," in Proceedings of the International Conference on Computational Science-Part III, ICCS 2002, (London, UK), pp. 105-114, 2002.

[127] A. Schinner, "Fast algorithms for the simulation of polygonal particles," Granul. Matter, vol. 2, no. 1, pp. 35-43, 1999.

[128] E. Perkins and J. Williams, "A fast contact detection algorithm insensitive to object sizes," Eng. Comput., vol. 18, no. 1-2, pp. 48-61, 2001.

[129] L. Pournin, On the behavior of spherical and non-spherical grain assemblies, its modeling and numerical simulation. PhD thesis, EPFL, Lausanne, France, 2005.

[130] L. Guibas and D. Russel, "An empirical comparison of techniques for updating delaunay triangulations," in Proceedings of the 20th annual symposium on Computational geometry, SCG 2004, pp. 170-179, 2004.

[131] B. C. Vemuri, Y. Cao, and L. Chen, "Fast collision detection algorithms with applications to particle flow," Comput. Graph. Forum, vol. 17, no. 2, pp. 121-134, 1998.

[132] L. Lu, Z. Gu, K. Lei, S. Wang, and K. Kase, "An efficient algorithm for detecting particle contact in non-uniform size particulate system," Particuology, vol. 8, no. 2, pp. $127-132,2010$.

[133] D. Quentrec and C. Brot, "New method for searching for neighbors in molecular dynamics computations," J. Comput. Phys., vol. 13, no. 3, pp. $430-432,1973$.

[134] A. Donev, F. H. Stillinger, and S. Torquato, "Neighbor list collision-driven molecular dynamics simulation for nonspherical particles. algorithmic details," J. Comput. Phys., vol. 202, no. 2, pp. 737-764, 2005.

[135] MATLAB, version 7.9.0 (R2009b). Natick, Massachusetts: The MathWorks Inc., 2009.

[136] T. Weinhart, A. Thornton, S. Luding, and O. Bokhove, "From discrete particles to continuum fields near a boundary," Granul. Matter, vol. 14, no. 2, pp. 289-294, 2012 . 
[137] D. Krijgsman and S. Luding, "2D cyclic pure shear of granular materials, simulations and model," AIP Conf. Proc., vol. 1542, no. 1, pp. 1226-1229, 2013.

[138] S. Chapman and T. G. Cowling, The mathematical theory of nonuniform gases. London: Cambridge University Press, 1960.

[139] J. M. Ziman, Models of Disorder. Cambridge: Cambridge University Press, 1979.

[140] T. Pöschel and S. Luding, eds., Granular Gases, (Berlin), Springer, 2001. Lecture Notes in Physics 564.

[141] A. Ferguson, B. Fisher, and B. Chakraborty, "Impulse distributions in dense granular flows: Signatures of large-scale spatial structures," EPL, vol. 66, no. 2, p. 277, 2004 .

[142] N. Rivas, S. Ponce, B. Gallet, D. Risso, R. Soto, P. Cordero, and N. Mujica, "Sudden chain energy transfer events in vibrated granular media," Phys. Rev. Lett., vol. 106, no. 8, p. 088001, 2011.

[143] L. D. Landau and E. M. Lifschitz, Physikalische Kinetik. Berlin: Akademie Verlag Berlin, 1986.

[144] J. G. Kirkwood, E. K. Maun, and B. J. Alder, "Radial distribution functions and the equation of state of a fluid composed of rigid spherical molecules," J. Chem. Phys., vol. 18, no. 8, pp. 1040-1047, 1950.

[145] R. J. Buehler, J. R. H. Wentorf, J. O. Hirschfelder, and C. F. Curtiss, "The free volume for rigid sphere molecules," J. Chem. Phys., vol. 19, no. 1, pp. 61-71, 1951.

[146] W. W. Wood, "Note on the free volume equation of state for hard spheres," J. Chem. Phys., vol. 20, p. 1334, 1952.

[147] Z. Salsburg and W. Wood, "Equation of state of classical hard spheres at high density," J. Chem. Phys., vol. 37, no. 4, p. 798, 1962.

[148] B. J. Alder, S. P. Frankel, and V. A. Lewinson, "Radial distribution function calculated by the Monte-Carlo Method for a hard sphere fluid," J. Chem. Phys., vol. 23, no. 3 , pp. $417-419,1955$.

[149] B. J. Alder and T. E. Wainwright, "Studies in molecular dynamics. I. General method.," J. Chem. Phys., vol. 31, no. 2, pp. 459-466, 1959.

[150] B. J. Alder and T. E. Wainwright, "Phase transition in elastic disks," Phys. Rev., vol. 127 , no. 2 , pp. 359-361, 1962.

[151] C. Rascón, L. Mederos, and G. Navascués, "Perturbation theory for classical solids," Phys. Rev. Lett., vol. 77, no. 11, pp. 2249-2252, 1996.

[152] B. Doliwa and A. Heuer, "Cage effect, local anisotropies, and dynamic heterogeneities at the glass transition: A computer study of hard spheres," Phys. Rev. Lett., vol. 80, pp. 4915-4918, 1998.

[153] S. Torquato, "Nearest-neighbor statistics for packings of hard spheres and disks," Phys. Rev. E, vol. 51, no. 4, pp. 3170-3182, 1995.

[154] E. Velasco and L. Mederos, "Equation of state of the hard-disc solid," Phys. Rev. $B$, vol. 56, no. 5, pp. 2432-2440, 1997.

[155] A. Santos, S. B. Yuste, and M. López de Haro, "Equation of state of a multicomponent $d$-dimensional hard sphere fluid," Molec. Phys., vol. 96, no. 1, pp. 1-5, 1999. 
[156] P. Richard, L. Oger, J.-P. Troadec, and A. Gervois, "Geometrical characterization of hard-sphere systems," Phys. Rev. E, vol. 60, no. 4, pp. 4551-4558, 1999.

[157] H. Löwen, "Fun with hard spheres," in Statistical Physics and Spatial Statistics (K. R. Mecke and D. Stoyan, eds.), (Berlin), Springer, 2000.

[158] S. Luding and O. Strauß, "The equation of state for almost elastic, smooth, polydisperse granular gases for arbitrary density," in Granular Gases (T. Pöschel and S. Luding, eds.), Lecture Notes in Physics 564, pp. 389-409, Berlin: Springer, 2000.

[159] S. Luding, "Liquid-solid transition in bi-disperse granulates," Adv. Complex Syst., vol. 4, no. 4, pp. 379-388, 2002.

[160] G. Parisi and F. Zamponi, "Mean-field theory of hard sphere glasses and jamming," Rev. Mod. Phys., vol. 82, no. 1, pp. 789-845, 2010.

[161] R. J. Speedy, "Quench rate independence of the hard sphere glass transition," Mol. Phys., vol. 83, no. 3, pp. 591-597, 1994.

[162] M. Hermes and M. Dijkstra, "Jamming of polydisperse hard spheres: The effect of kinetic arrest," EPL, vol. 89, no. 3, p. 38005, 2010.

[163] R. S. Farr and R. D. Groot, "Close packing density of polydisperse hard spheres," J. Chem. Phys., vol. 131, no. 24, p. 244104, 2009.

[164] M. Clusel, E. I. Corwin, A. O. N. Siemens, and J. Brujic, "A 'granocentric' model for random packing of jammed emulsions," Nature, vol. 460, no. 7255, pp. 611-615, 2009.

[165] I. Biazzo, F. Caltagirone, G. Parisi, and F. Zamponi, "Theory of Amorphous Packings of Binary Mixtures of Hard Spheres," Phys. Rev. Lett., vol. 102, no. 19, p. $195701,2009$.

[166] M. Danisch, Y. Jin, and H. A. Makse, "Model of random packings of different size balls," Phys. Rev. E, vol. 81, no. 5, p. 051303, 2010.

[167] K. A. Newhall, I. Jorjadze, E. Vanden-Eijnden, and J. Brujic, "A statistical mechanics framework captures the packing of monodisperse particles," Soft Matter, vol. 7, no. 24 , pp. 11518-11525, 2011.

[168] J. Jenkins and F. Mancini, "Balance Laws and Constitutive Relations for Plane Flows of a Dense, Binary Mixture of Smooth, Nearly Elastic, Circular Disks," J. Appl. Mech.-Trans. ASME, vol. 54, no. 1, pp. 27-34, 1987.

[169] E. W. Grundke and D. Henderson, "Distribution functions of multi-component fluid mixtures of hard spheres," Mol. Phys., vol. 24, no. 2, p. 269, 1972.

[170] L. L. Lee and D. Levesque, "Perturbation theory for mixtures of simple liquids," Mol. Phys., vol. 26, no. 6, pp. 1351-1370, 1973.

[171] N. Carnahan and K. Starling, "Equation of state for non-attracting rigid spheres," J. Chem. Phys., vol. 51, no. 2, pp. 635-636, 1969.

[172] S. Torquato and S. B. Lee, "Computer simulations of nearest-neighbor distribution functions and related quantities for hard-sphere systems," Physica A, vol. 167, no. 2, pp. 361-383, 1990.

[173] R. D. Kamien and A. J. Liu, "Why is random close packing reproducible?," Phys. Rev. Lett., vol. 99, no. 15, p. 155501, 2007. 
[174] T. Boublík and I. Nezbeda, "P-V-T Behaviour of Hard Body Fluids. Theory and Experiment.," Collect. Czech. Chem. Commun., vol. 51, no. 11, pp. 2301-2432, 1986.

[175] T. Boublík, "Equation of State of Hard Body Fluids," Mol. Phys., vol. 59, no. 2, pp. 371-380, 1986.

[176] J. V. Sengers, R. F. Kayser, C. J. Peters, and H. J. White, Equations of State for Fluids and Fluid Mixtures (Experimental Thermodynamics). Amsterdam: Elsevier Science, 2000.

[177] A. H. Alexopoulos and C. Kiparissides, "On the prediction of internal particle morphology in suspension polymerization of vinyl chloride. Part I: The effect of primary particle size distribution," Chem. Eng. Sci., vol. 62, no. 15, pp. 3970-3983, 2007.

[178] D. Ballal and A. Lefebvre, "Ignition and flame quenching of flowing heterogeneous fuel-air mixtures," Combust. Flame, vol. 35, no. 2, pp. 155-168, 1979.

[179] A. Santos, S. B. Yuste, and M. López de Haro, "Communication: Inferring the equation of state of a metastable hard-sphere fluid from the equation of state of a hard-sphere mixture at high densities," J. Chem. Phys., vol. 135, no. 18, p. 181102, 2011.

[180] A. Kansal, S. Torquato, and F. Stillinger, "Computer generation of dense polydisperse sphere packings," J. Chem. Phys., vol. 117, no. 18, pp. 8212-8218, 2002.

[181] A. Donev, S. Torquato, and F. H. Stillinger, "Pair correlation function characteristics of nearly jammed disordered and ordered hard-sphere packings," Phys. Rev. E, vol. 71 , no. 1, p. $011105,2005$.

[182] E. Zaccarelli, C. Valeriani, E. Sanz, W. C. K. Poon, M. E. Cates, and P. N. Pusey, "Crystallization of Hard-Sphere Glasses," Phys. Rev. Lett., vol. 103, no. 13, p. 135704,2009 .

[183] W. van Megen, S. I. Henderson, S. R. Williams, and I. K. Snook, "Crystallisation and Metastability of Binary Fluids," in Statistical Physics: Experiments, Theories and Computer simulations (M. Tokuyama and I. Oppenheim, ed.), pp. 14-17, World Scientific, Singapore, 1998.

[184] P. Sollich and N. B. Wilding, "Crystalline Phases of Polydisperse Spheres," Phys. Rev. Lett., vol. 104, no. 11, p. 118302, 2010.

[185] R. Speedy, "Pressure and entropy of hard-sphere crystals," J. Phys.: Condens. Mat., vol. 10, no. 20, pp. 4387-4391, 1998.

[186] R. Speedy, "Pressure of the metastable hard-sphere fluid," J. Phys.: Condens. Mat., vol. 9, no. 41, pp. 8591-8599, 1997.

[187] J. Tobochnik and P. M. Chapin, "Monte Carlo simulation of hard spheres near random closest packing using spherical boundary conditions," J. Chem. Phys., vol. 88, no. 9 , pp. 5824-5830, 1988 .

[188] S. R. Williams, C. P. Royall, and G. Bryant, "Crystallization of dense binary hard-sphere mixtures with marginal size ratio," Phys. Rev. Lett., vol. 100, no. 22, p. 225502,2008 .

[189] N. Hunt, R. Jardine, and P. Bartlett, "Superlattice formation in mixtures of hardsphere colloids," Phys. Rev. E, vol. 62, no. 1, pp. 900-913, 2000.

[190] J. V. Sanders, "Close-packed structures of spheres of two different sizes. I. Observations on natural opal," Philos. Mag. A, vol. 42, no. 6, pp. 705-720, 1980. 
[191] S. Hachisu and S. Yoshimura, "Optical demonstration of crystalline superstructures in binary mixtures of latex globules," Nature, vol. 283, no. 5743, pp. 188-189, 1980.

[192] A. B. Schofield, P. N. Pusey, and P. Radcliffe, "Stability of the binary colloidal crystals $a B_{2}$ and $a B_{13}$," Phys. Rev. E, vol. 72, no. 3, p. 031407, 2005.

[193] F. Göncü, O. Duran, and S. Luding, "Constitutive relations for the isotropic deformation of frictionless packings of polydisperse spheres," C. R. Mecanique, vol. 338, no. $10-11$, pp. 570-586, 2010.

[194] H. J. Herrmann, R. M. Baram, and M. Wackenhut, "Polydisperse packings," Braz. J. Phys., vol. 33, no. 3, pp. 591-593, 2003.

[195] T. Aste and A. Coniglio, "Cell theory for liquid solids and glasses: From local packing configurations to global complex behaviors," Europhys. Lett., vol. 67, no. 2, pp. $165-171,2004$.

[196] A. B. Hopkins, Y. Jiao, F. H. Stillinger, and S. Torquato, "Phase diagram and structural diversity of the densest binary sphere packings," Phys. Rev. Lett., vol. 107, no. 12 , p. 125501, 2011.

[197] L. V. Woodcock and C. A. Angell, "Diffusivity of the hard-sphere model in the region of fluid metastability," Phys. Rev. Lett., vol. 47, no. 16, pp. 1129-1132, 1981.

[198] J. Bernal, "A Geometrical Approach to the Structure Of Liquids," Nature, vol. 183, no. 4655 , pp. 141-147, 1959.

[199] J. G. Berryman, "Random close packing of hard spheres and disks," Phys. Rev. A, vol. 27, no. 2, pp. 1053-1061, 1983.

[200] W. S. Xu, Z. Y. Sun, and L. J. An, "Dense packing in the monodisperse hard-sphere system: A numerical study," Eur. Phys. J. E, vol. 31, no. 4, pp. 377-382, 2010.

[201] S. Torquato and F. H. Stillinger, "Jammed hard-particle packings: From kepler to bernal and beyond," Rev. Mod. Phys., vol. 82, no. 3, pp. 2633-2672, 2010.

[202] V. Ogarko, N. Rivas, and S. Luding, "Structure characterization of hard sphere packings in amorphous and crystalline states." Submitted, 2014.

[203] C. Hrenya. private communications, 2011.

[204] P. Coles and B. Jones, "A lognormal model for the cosmological mass distribution," Mon. Not. R. Astr. Soc., vol. 248, no. 1, pp. 1-13, 1991.

[205] N. Xu, T. K. Haxton, A. J. Liu, and S. R. Nagel, "Equivalence of Glass Transition and Colloidal Glass Transition in the Hard-Sphere Limit," Phys. Rev. Lett., vol. 103, no. 24, p. 245701, 2009.

[206] M. Schmiedeberg, T. K. Haxton, S. R. Nagel, and A. J. Liu, "Mapping the glassy dynamics of soft spheres onto hard-sphere behavior," EPL, vol. 96, no. 3, p. 36010, 2011.

[207] R. M. L. Evans, D. J. Fairhurst, and W. C. K. Poon, "Universal law of fractionation for slightly polydisperse systems," Phys. Rev. Lett., vol. 81, no. 6, pp. 1326-1329, 1998.

[208] K. Gotoh and J. L. Finney, "Statistical geometrical approach to random packing density of equal spheres," Nature, vol. 252, no. 5480, pp. 202-205, 1974.

[209] E. I. Corwin, M. Clusel, A. O. N. Siemens, and J. Brujic, "Model for random packing of polydisperse frictionless spheres," Soft Matter, vol. 6, no. 13, pp. 2949-2959, 2010. 
[210] J. K. Percus and G. J. Yevick, "Analysis of classical statistical mechanics by means of collective coordinates," Phys. Rev., vol. 110, no. 1, pp. 1-13, 1958.

[211] J. L. Lebowitz, "Exact solution of generalized percus-yevick equation for a mixture of hard spheres," Phys. Rev., vol. 133, no. 4A, pp. A895-A899, 1964.

[212] Y. Rosenfeld, "Free-energy model for the inhomogeneous hard-sphere fluid mixture and density-functional theory of freezing," Phys. Rev. Lett., vol. 63, no. 9, pp. 980983, 1989.

[213] M. Fasolo and P. Sollich, "Equilibrium phase behavior of polydisperse hard spheres," Phys. Rev. Lett., vol. 91, no. 6, p. 068301, 2003.

[214] R. M. Baram, H. J. Herrmann, and N. Rivier, "Space-filling bearings in three dimensions," Phys. Rev. Lett., vol. 92, no. 4, p. 044301, 2004.

[215] A. Donev, F. H. Stillinger, and S. Torquato, "Configurational entropy of binary harddisk glasses: Nonexistence of an ideal glass transition," J. Chem. Phys., vol. 127, no. 12 , p. 124509, 2007.

[216] L. V. Woodcock, "Thermodynamic description of liquid-state limits," J. Phys. Chem. B, vol. 116, no. 12, pp. 3735-3744, 2012.

[217] P. R. ten Wolde, M. J. Ruiz-Montero, and D. Frenkel, "Simulation of homogeneous crystal nucleation close to coexistence," Faraday Discuss., vol. 104, pp. 93-110, 1996.

[218] L. T. DeCarlo, "On the meaning and use of kurtosis," Psychol. Methods, vol. 2, no. 3, pp. 292-307, 1997.

[219] M. R. Shaebani, M. Madadi, S. Luding, and D. E. Wolf, "Influence of polydispersity on micromechanics of granular materials," Phys. Rev. E, vol. 85, no. 1, p. 011301, 2012.

[220] D. Bi, J. Zhang, B. Chakraborty, and R. P. Behringer, "Jamming by shear," Nature, vol. 480, no. 7377, pp. 355-358, 2011.

[221] A. Clauset, C. Shalizi, and M. Newman, "Power-law distributions in empirical data," SIAM Rev., vol. 51, no. 4, pp. 661-703, 2009.

[222] R. S. Farr, "Random close packing fractions of lognormal distributions of hard spheres," Powder Technol., vol. 245, pp. 28 - 34, 2013.

[223] J. Söderlund, L. B. Kiss, G. A. Niklasson, and C. G. Granqvist, "Lognormal size distributions in particle growth processes without coagulation," Phys. Rev. Lett., vol. 80 , no. 11 , pp. $2386-2388,1998$.

[224] P. J. Steinhardt, D. R. Nelson, and M. Ronchetti, "Bond-orientational order in liquids and glasses," Phys. Rev. B, vol. 28, no. 2, pp. 784-805, 1983.

[225] P. R. ten Wolde, M. J. Ruiz-Montero, and D. Frenkel, "Numerical evidence for bcc ordering at the surface of a critical fcc nucleus," Phys. Rev. Lett., vol. 75, no. 14, pp. 2714-2717, 1995.

[226] A. B. Hopkins, F. H. Stillinger, and S. Torquato, "Nonequilibrium static diverging length scales on approaching a prototypical model glassy state," Phys. Rev. E, vol. 86, no. 2, p. 021505, 2012.

[227] É. Marcotte, F. H. Stillinger, and S. Torquato, "Nonequilibrium static growing length scales in supercooled liquids on approaching the glass transition," J. Chem. Phys., vol. 138, no. 12, p. 12A508, 2013. 
[228] C. E. Zachary, Y. Jiao, and S. Torquato, "Hyperuniform long-range correlations are a signature of disordered jammed hard-particle packings," Phys. Rev. Lett., vol. 106, no. 17, p. 178001, 2011.

[229] S. Sýkora, "K-Space Images of n-Dimensional Spheres and the Generalized Sinc Functions," in Stan's Library, Vol. II (S. Sýkora, ed.), 2007. DOI:10.3247/SL2Math07.002.

[230] J. D. Bernal, "Geometry of the structure of monatomic liquids," Nature, vol. 185, no. 4706 , pp. $68-70,1960$.

[231] W. B. Russel, D. A. Saville, and W. R. Schowalter, Colloidal Dispersions. Cambridge University Press, 1992.

[232] J. Duran, Sands, powders, and grains: an introduction to the physics of granular materials. New York: Springer, 2000.

[233] M. H. Kalos, D. Levesque, and L. Verlet, "Helium at zero temperature with hardsphere and other forces," Phys. Rev. A, vol. 9, no. 5, pp. 2178-2195, 1974.

[234] S. Torquato and F. Lado, "Effective properties of two-phase disordered composite media: II. evaluation of bounds on the conductivity and bulk modulus of dispersions of impenetrable spheres," Phys. Rev. B, vol. 33, no. 9, pp. 6428-6435, 1986.

[235] B. O'Malley and I. Snook, "Crystal nucleation in the hard sphere system," Phys. Rev. Lett., vol. 90, no. 8, p. 085702, 2003.

[236] M. S. Wertheim, "Exact solution of the percus-yevick integral equation for hard spheres," Phys. Rev. Lett., vol. 10, no. 8, pp. 321-323, 1963.

[237] C. D. Estrada and M. Robles, "Fluidsolid transition in hard hypersphere systems," J. Chem. Phys., vol. 134, no. 4, p. 044115, 2011.

[238] J. L. Finney and J. Wallace, "Interstice correlation functions; a new, sensitive characterisation of non-crystalline packed structures," J. Non-Cryst. Solids, vol. 43, no. 2, pp. 165-187, 1981.

[239] R. P. Bywater and J. L. Finney, "Particle geometry and the chromatographic separation of cells: The simplest case of uniform particles," J. Theor. Biol., vol. 105, no. 2, pp. $333-343,1983$.

[240] Y. Hiwatari, T. Saito, and A. Ueda, "Structural characterization of soft-core and hard-core glasses by delaunay tessellation," J. Chem. Phys., vol. 81, no. 12, pp. 6044$6050,1984$.

[241] M. Kimura and F. Yonezawa, "Nature of amorphous and liquid structures computer simulations and statistical geometry," J. Non-Cryst. Solids, vol. 6162, Part 1, pp. 535-540, 1984 .

[242] S. L. Chan and S. R. Elliott, "Interstices, diffusion doorways and free volume percolation studies of a dense random packed atomic structure," J. Non-Cryst. Solids, vol. 124 , no. 1 , pp. $22-33,1990$.

[243] V. Voloshin, S. Beaufils, and N. Medvedev, "Void space analysis of the structure of liquids," J. Mol. Liq., vol. 9697, pp. 101-112, 2002.

[244] A. P. Philipse, "Caging effects in amorphous hard-sphere solids," Colloid. Surface $A$, vol. 213, no. 23, pp. 167-173, 2003. 
[245] M. D. Haw, "Void structure and cage fluctuations in simulations of concentrated suspensions," Soft Matter, vol. 2, no. 11, pp. 950-956, 2006.

[246] A. V. Anikeenko, M. L. Gavrilova, and N. N. Medvedev, "Shapes of delaunay simplexes and structural analysis of hard sphere packings," in Generalized Voronoi Diagram: A Geometry-Based Approach to Computational Intelligence (M. L. Gavrilova, ed.), no. 158 in Studies in Computational Intelligence, pp. 13-45, Springer Berlin Heidelberg, 2008.

[247] R. Courant and H. Robbins, "Apollonius' problem.," in What Is Mathematics?: An Elementary Approach to Ideas and Methods, pp. 117 and 125-127, Oxford, England: Oxford University Press, 1996.

[248] A. Santos, S. B. Yuste, M. López de Haro, G. Odriozola, and V. Ogarko, "A simple effective rule to estimate the jamming packing fraction of polydisperse hard spheres." Submitted, arXiv:1402.3443, 2014.

[249] V. Ogarko, S. Luding, and W. T. Kranz, "Rapidly quenched inelastic hard spheres: Glass transition and jamming." In preparation, 2014.

[250] V. P. Il'in, D. V. Knysh, and V. A. Ogarko, "On the efficiency of parallelization of conjugate direction and semi-conjugate direction methods (in Russian)," in Proceedings of the international conference "Parallel Computing Technologies", vol. 1, (Chelyabinsk, Russia), pp. 244-255, 2007. 


\section{Curriculum vitae}

\section{Vitaliy Ogarko}

Born on May $5^{\text {th }}, 1985$ in Vladivostok, Russia.

Jun. 2002 High School Diploma,

Specialized Educational Scientific Center of

Novosibirsk State University, Russia.

Jun. 2006

BSc in Mathematics,

Novosibirsk State University, Russia.

Jun. 2008

MSc in Applied Mathematics and Informatics, Novosibirsk State University, Russia.

Sep. 2008 - Mar. 2009 Internship,

Schlumberger K.K., Japan.

Jun. 2009 - Feb. 2014 PhD candidate,

University of Twente, The Netherlands.

Mar. 2014 - present Software Engineer at TomTom,

Amsterdam, The Netherlands.

Publications

Journal Papers

1. V. Ogarko, N. Rivas, and S. Luding, "Structure characterization of hard sphere packings in amorphous and crystalline states." Submitted, 2014.

2. A. Santos, S. B. Yuste, M. López de Haro, G. Odriozola, and V. Ogarko, "A simple effective rule to estimate the jamming packing fraction of polydisperse hard spheres." Submitted, arXiv:1402.3443, 2014.

3. D. Krijgsman, V. Ogarko, and S. Luding, "Optimal parameters for a hierarchical grid data structure for contact detection in arbitrarily polydisperse particle systems." Accepted in Comp. Part. Mech., 2014.

4. V. Ogarko and S. Luding, "Prediction of polydisperse hard-sphere mixture behavior using tridisperse systems," Soft Matter, vol. 9, no. 40, pp. 9530-9534, 2013. This research was featured on the inside cover. 
5. A. R. Thornton, T. Weinhart, V. Ogarko, and S. Luding, "Multi-scale methods for multi-component granular materials," Comput. Meth. in Mater. Sci., vol. 13, no. 2, pp. 197-212, 2013.

6. V. Ogarko and S. Luding, "Equation of state and jamming density for equivalent bi- and polydisperse, smooth, hard sphere systems," $J$. Chem. Phys., vol. 136, no. 12, p. 124508, 2012.

7. V. Ogarko and S. Luding, "A fast multilevel algorithm for contact detection of arbitrarily polydisperse objects," Comput. Phys. Commun., vol. 183, no. 4, pp. 931-936, 2012.

8. V. Ogarko, S. Luding, and W. T. Kranz, "Rapidly quenched inelastic hard spheres: Glass transition and jamming." In preparation, 2014.

Proceedings

1. A. R. Thornton, D. Krijgsman, A. te Voortwis, V. Ogarko, S. Luding, R. Fransen, S. González, O. Bokhove, O. Imole, and T. Weinhart, "A review of recent work on the discrete particle method at the university of twente: An introduction to the open-source package mercurydpm," in Proc. 6th Int. Conf. DEM (DEM6), (Golden, CO, USA), pp. 50-56, 2013.

2. V. Ogarko and S. Luding, "A study on the influence of the particle packing fraction on the performance of a multilevel contact detection algorithm," in II International Conference on Particle-based Methods - Fundamentals and Applications (E. Oñate and D. R. J. Owen, eds.), (Barcelona, Spain), pp. 1-7, 2011.

3. V. Ogarko and S. Luding, "Data structures and algorithms for contact detection in numerical simulation of discrete particle systems," in Proceedings of the 6th World Congress on Particle Technology (WCPT6), (Nuremberg, Germany), 2010.

4. V. P. Il'in, D. V. Knysh, and V. A. Ogarko, "On the efficiency of parallelization of conjugate direction and semi-conjugate direction methods (in Russian)," in Proceedings of the international conference "Parallel Computing Technologies", vol. 1, (Chelyabinsk, Russia), pp. 244-255, 2007. 


\section{Curriculum vitae}

Presentations

1. $6^{\text {th }}$ International Conference on Discrete Element Methods (DEM6), Golden, Colorado, USA, Aug. 2013.

2. APS March Meeting 2013, abs. J30.011, Baltimore, USA, Mar. 2013.

3. Int. Workshop on Packing Problems, Dublin, Ireland, Sept. 2012.

4. MuST Days, Utrecht, The Netherlands, Apr. 2012.

5. APS March Meeting 2012, abs. W53.009, Boston, MA, USA, Mar. 2012.

6. II Int. Conf. on Particle-based Methods, Barcelona, Spain, Oct. 2011.

7. Workshop Granular and Active Fluids, Zaragoza, Spain, Sept. 2011.

8. $13^{\text {th }}$ Eng. Mech. Symposium, Lunteren, The Netherlands, Oct. 2010.

9. $6^{\text {th }}$ World Congress Particle Technology (WCPT6) (Poster), Nuremberg, Germany, Mar. 2010. 

